Federal Reserve Bank of Minneapolis

Research Department

\title{
COMPUTATION AND MULTIPLICITY
}

OF EQUILIBRIA

Timothy f. Kehoe*

Working Paper 460

Revised October 1991

\begin{abstract}
Economic equilibria are usually solutions to fixed point problems rather than solutions to convex optimization problems. This leads to two difficulties that are closely related: First, equilibria may be difficult to compute. Second, a model economy may have more than one equilibrium. This paper explores these issues for a number of stylized economies, including static economies that involve both pure exchange and production, economies that have infinite numbers of goods because of time and uncertainty, and economies with distortionary taxes and externalities. There are numerous numerical examples that illustrate the theory and could serve as test problems for algorithms.
\end{abstract}

*University of Minnesota and Federal Reserve Bank of Minneapolis. This paper has been prepared for Volume IV of Handbook of Mathematical Economics edited by $\mathrm{H}$. Sonnenschein and W. Hildenbrand. I would like to thank participants in BoWo 89 for helpful comments and suggestions, especially Robert Anderson, Donald Brown, Gerard Debreu, Hildegard Dierker, Michael Jerison, Reinhard John, Andreu Mas-Colell, Harald Uhlig, and William Zame. I gratefully acknowledge support from Deutsche Forschungsgemeinshaft, Gottfried-Wilheim-Leibniz-Forderpreis during BoWo 89 and from National Science Foundation grants SES 87-08616 and SES 89-22036.

The views expressed herein are those of the author and not necessarily those of the Federal Reserve Bank of Minneapolis or the Federal Reserve System. 


\section{Introduction}

In recent years the Walrasian general equilibrium model has become an important tool for applied work in such fields as development economics, international trade, macroeconomics, and public finance (see, for example, Shoven and Whalley 1984 and Lucas 1987). Unfortunately, economic equilibria are usually solutions to fixed point problems, rather than solutions to convex optimization problems. This leads to two difficulties that are closely related: First, equilibria may be difficult to compute. Second, a model economy may have more than one equilibrium.

In this paper we explore these two issues for a number of stylized economies. We start with static exchange economies and then add a production technology. We later analyze economies with infinite numbers of goods, economies in which time and uncertainty play important roles. Studying economies of this sort is interesting not only for its own sake but because of the insights that it provides into the properties of economies with large, but finite numbers of goods. Finally, we extend our analysis to economies that include distortionary taxes and externalities.

Although our emphasis is on computational issues, the approach is fairly theoretical. As Scarf $(1973,1982)$ has stressed, a precondition for developing computational methods for finding an equilibrium is to know that one exists. Furthermore, existence proofs often suggest algorithms for computing equilibria. Consequently, much of this paper deals with proofs of the existence of equilibria for a variety of economies. The paper is also filled with simple numerical examples, however, that illustrate the theory and could serve as test problems for algorithms. The reader who wishes to implement any of the algorithms suggested here on the computer should consult a good book on numerical methods. Two are: Acton (1970), who provides a wealth of practical advice, and Press, Flannery, Teukolsky, and Vetterling (1986), who provide a large number of useful computer programs. 


\section{Static Exchange Economies}

We begin by reviewing results for exchange economies with a finite number of consumers and a finite number of goods. We use two alternative specifications of such an economy, the first in terms of utility functions and endowment vectors, the second in terms of aggregate excess demand functions. Although the first specification is in some sense the more primitive, we alternate between the two as is convenient.

\subsection{Existence of Equilibrium}

Consider an economy with $\mathrm{m}$ consumers and $\mathrm{n}$ goods. Each consumer has preferences given by a utility function $u_{i}: R_{+}^{n} \rightarrow R$ that is strictly concave and monotonically increasing. He is endowed with a vector $w^{i}=\left(w_{1}^{i}, \ldots, w_{n}^{i}\right)$ that is strictly positive. An equilibrium of this economy is a price vector $\hat{p} \in \mathbf{R}_{+}^{\mathrm{n}} \backslash\{0\}$ and an allocation $\left(\hat{x}^{1}, \ldots, \mathrm{x}^{\mathrm{m}}\right)$, where $\hat{x}^{\mathrm{i}} \in \mathrm{R}_{+}^{\mathrm{n}}$, such that

- $\mathrm{x}^{\mathrm{i}}, \mathrm{i}=1, \ldots, \mathrm{m}$, solves $\max u_{i}(x)$

subject to

$$
\begin{aligned}
& \hat{\rho}^{\prime} x \leq \beta^{\prime} w^{i} \\
& x \geq 0 \\
& -\sum_{i=1}^{m} x^{i} \leq \sum_{i=1}^{m} w^{i} .
\end{aligned}
$$

Alternatively, we could specify this economy in terms of an aggregate excess demand function $f: R_{+}^{n} \backslash\{0\} \rightarrow R^{n}$. We assume that $f$ is continuous; that it is homogeneous of degree zero, $f(\theta p)=f(p)$ for all $\theta>0$ and all $p \in R_{+}^{n} \backslash\{0\}$; and that is obeys Walras's law, $p^{\prime} f(p) \equiv 0$ for all $p \in R_{+}^{n} \backslash\{0\}$. An equilibrium is now a price vector $\hat{p} \in R_{+}^{n} \backslash\{0\}$ such that

- $\mathrm{f}(\mathrm{p}) \leq 0$. 
Notice that Walras's law implies that $\mathrm{f}_{\mathrm{i}}(\boldsymbol{\beta})=0$ if $\hat{\mathrm{p}}_{\mathrm{i}}>0$.

To motivate our assumptions on $f$, we can think of $f$ as being derived from the first specification of this economy. Let $x^{i}(p)$ be the solution to the consumer's utility maximization problem. Our assumptions on $u_{i}$ and $w^{i}$ imply that $x^{i}$ is continuous, at least for all strictly positive price vectors; that it is homogeneous of degree zero, $x^{i}(\theta p) \equiv x^{i}(p)$ for all $\theta>0, p \in R_{++}^{n}$; and that it obeys the budget constraint, $p^{\prime} x^{i}(p) \equiv p^{\prime} w^{i}$. The aggregate excess demand function

$$
f(p)=\sum_{i=1}^{m}\left(x^{i}(p)-w^{i}\right)
$$

therefore, is continuous, at least at all strictly positive price vectors, is homogeneous of degree zero, and obeys Walras's law.

Unfortunately, utility maximization does not imply that $f$ is continuous on all $R_{+}^{n} \backslash\{0\}$. Rather, it implies that $f$ is continuous and bounded below on all $R_{++}^{\mathrm{n}}$. For some, but not necessarily all, $p^{0} \in R_{+}^{n} \backslash\{0\}$ where $p_{i}^{0}=0$, some $i$, if $p^{k} \rightarrow p^{0}, p^{k} \in R_{++}^{n}$, then $\left\|f\left(p^{k}\right)\right\| \rightarrow \infty$. (See, for example, Arrow and Hahn 1971, Chapter 2.) One way to handle the possibility that f becomes unbounded at price vectors that have some zero elements is to impose a constraint like $\mathrm{x} \leq$ $2 \sum \sum_{j=1}^{m} w^{j}$, in each consumer's utility maximization problem. With such a constraint $x^{i}(p)$, and therefore $f$, is continuous on all $R_{+}^{n} \backslash\{0\}$. Furthermore, such a constraint cannot bind in equilibrium. An alternative way to handle this possibility is to show that, for any $f: R_{++}^{n} \rightarrow R$ that satisfies the conditions implied by utility maximization, there is another function $\mathrm{f}^{*}$ that satisfies our assumptions, agrees with $f$ on some open neighborhood of every equilibrium of $f$, and has no equilibria that are not equilibria of $f$ (see, for example, Kehoe 1982). In any case, since the potential unboundedness of excess demand when some prices are zero does not play a significant conceptual role in any of the issues discussed in this paper, we shall ignore it. 
There is a close connection between equilibria of such economies and fixed points of continuous mappings of the simplex $S=\left\{p \in R_{+}^{n} \mid e^{t} p=1, p \geq 0\right\}$ into itself. (Here and subsequently, $e=(1, \ldots, 1)$.) To prove the existence of equilibrium we employ Brouwer's fixed point theorem.

Brouwer's Fixed Point Theorem. Let $S \subset \mathrm{R}^{\mathrm{n}}$ be any nonempty, compact, convex set, and let $g: S \rightarrow S$ be continuous. Then $g$ leaves some $x \in S$ fixed, that is, $x=g(x)$.

Homogeneity allows us to restrict our attention in studying equilibria to the simplex: if $f(\hat{p}) \leq 0$ for $\hat{p} \in S$, then $f(\bar{p}) \leq 0$ for $\vec{p}=\hat{p} /\left(e^{\prime} \hat{p}\right)$. S is nonempty, compact, and convex. Consider the function $g: S \rightarrow S$ that associates with any point $p \in S$ the point $g(p) \in S$ that is the closest point in $S$ to $p+f(p)$ in terms of euclidean distance. In other words, $g(p)$ solves the problem

$$
\min (1 / 2)(g-p-f(p))^{\prime}(g-p-f(p))
$$

subject to

$$
\begin{aligned}
& \mathrm{e}^{\prime} \mathrm{g}=1 \\
& \mathrm{~g} \geq 0
\end{aligned}
$$

Since the objective function is strictly convex in $\mathrm{g}$ and continuous in $\mathrm{p}$ and the constraint set is convex, $g(p)$ is a continuous function.

Proposition 2.1. $p$ is an equilibrium of $f$ if and only if it is a fixed point of $g, \hat{p}=g(\hat{p})$.

Proof. $g(p)$ solves the minimization problem that defines it if and only if there exists $\lambda \in R$ such that

$$
\begin{aligned}
& g(p)-p-f(p)-\lambda e \geq 0 \\
& g(p)^{\prime}(g(p)-p-f(p)-\lambda e)=0 .
\end{aligned}
$$


If $g(\hat{\theta})=\hat{p}$, then the second, the complementary slackness, condition becomes $-\hat{p}^{\prime}(f(\hat{p})+\hat{\lambda} e)=\hat{\lambda} \hat{p}^{\prime} e=\hat{\lambda}=0$.

The first condition then becomes $f(p) \leq 0$.

Conversely, if $\hat{p}$ is an equilibrium, we set $\lambda=0$ and observe that $\hat{p}$ satisfies the above conditions that define $\mathrm{g}(\mathrm{p})$.

Bemark. This result can easily be extended to economies where excess demand is a nonempty, bounded, upper-hemi-continuous, convex-valued correspondence rather than a continuous, single-valued function. We need to alter $g$, however, because, as defined above, $g(p)$ is not necessarily convex-valued. Let $D$ be the convex hull of the image of $p+f(p)$ for $p \in S$. Since $f$ is bounded and upper-hemi-continuous and $S$ is compact, $D$ is compact. Let $q: D \rightarrow S$ be the function that associates any point $p \in D$ with the point $q(p) \in S$ that is closest to $p$. Now let $g: D \rightarrow D$ be the correspondence $g(p)=q(p)+f(q(p))$. In other words, instead of first adding excess demand and then projecting into the simplex, we first project into the simplex and then add excess demand. The advantage is that $\mathrm{g}$ is now convex-valued as well as being upper-hemi-continuous. By Kakutani's fixed point theorem, it has a fixed point $\hat{p} \in g(\hat{p})$. An easy argument, similar to that above, implies that $p$ is a fixed point if and only if it is an equilibrium. In practice, however, working with a domain like $\mathrm{D}$ is often more difficult than with one like $S$.

The above result is useful because it allows us to reduce the problems of computing equilibria and establishing the uniqueness of equilibrium to the analogous problems for fixed points. The question arises whether this is the easiest approach. In many circumstances it is. If the only assumptions that we make on $f$ are those of continuity, homogeneity, and Warras's law, then an argument due to Uzawa (1962) says that the study of equilibria is in a sense, equivalent to the study of fixed points. Suppose that $\gamma: S \rightarrow S$ is continuous. Consider the artificial excess demand function 
$\phi: \mathbb{R}_{+}^{\mathrm{n}} \backslash\{0\} \rightarrow \mathrm{R}^{\mathrm{n}}$ defined by $\phi(\mathrm{p})=\gamma\left(\mathrm{p} /\left(\mathrm{e}^{\prime} \mathrm{p}\right)\right)-\lambda(\mathrm{p}) \mathrm{p}$, where $\lambda(\mathrm{p})=\gamma\left(\mathrm{p} /\left(\mathrm{e}^{\prime} \mathrm{p}\right)\right)^{\prime} \mathrm{p} /\left(\mathrm{p}^{\prime} \mathrm{p}\right) . \quad$ By construction, $\phi$ is continuous, is homogeneous of degree zero, and obeys Walras's law.

Propesition 2.2 (Uzawa 1962). $p$ is a fixed point of $\gamma$ if only it is an equilibrium of $\phi$, that is, $\phi(\hat{p}) \leq 0$ and $\hat{p} \in S$.

Proof. If $\phi(\beta) \leq 0$ and $p \in S$, then

$$
\gamma_{i}(p)-\lambda(\hat{p}) \hat{p}_{i} \leq 0, \quad i=1, \ldots, n .
$$

Since Walras's law implies $\phi_{i}(\hat{p})<0$ only if $\hat{p}_{i}=0$ and since $\gamma_{i}(\hat{p}) \geq 0$, this can be rewritten as

$$
\lambda(\hat{p}) \hat{p}_{i}=\gamma_{i}(\hat{p}), \quad \mathrm{i}=1, \ldots, \mathrm{n} .
$$

Consequently, $\mathrm{e}^{\prime} \hat{\beta}=\mathrm{e}^{\prime} \gamma(\hat{\beta})=1$ implies $\hat{\mathrm{p}}=\gamma(\beta)$.

Conversely, if $\hat{\beta}=\gamma(\hat{\beta})$ then $\lambda(\hat{\beta})=1$ and $\phi(\hat{\beta})$.

Remark. Suppose that we are willing to impose no stronger conditions on $u_{i}$ and $w^{i}$ than those given previously and that $m \geq n$, in other words, that there are at least as many consumers as goods. Then a series of results due to Sonnenschein (1973); Mantel (1974); Debreu (1974); McFadden, Mas-Colell, Mantel, and Richter (1974); and Mas-Colell (1977) says that the only assumptions that we are justified in imposing on $f$ are continuity, homogeneity, and Walras's law: for any $f$ that satisfies these assumptions there is an economy with $\mathrm{n}$ consumers whose excess demands aggregate to a function $f^{*}$ whose equilibria coincide with those of $f$ and that agrees with $f$ on any compact set of prices where $\mathrm{f}$ is continuous (see Shafer and Sonnenschein 1982). Imposing stronger restrictions on $u_{i}$ and $w^{i}$, however, can sometimes make the study of equilibria easier than the study of fixed points. 
Interpretations of Uzawa's result are delicate. It is not surprising that we can show that the equilibrium existence theorem holds if and only if Brouwer's fixed point theorem does, since both are theorems given the axioms of modern mathematics. What is important is how easy and trivial the result is: Any theorem that proves the existence of a fixed point can be translated into a theorem that proves the existence of equilibrium by adding a few lines to the proof, and conversely. Any computer program that computes fixed points can be translated into a program that computes equilibria by adding a few lines to the code, and conversely. Any conditions that imply uniqueness of a fixed point can be easily translated into conditions that imply uniqueness of equilibrium, and conversely.

There is an important caveat to this interpretation of Uzawa's result: It relates fixed points to equilibria of economies specified in terms of aggregate excess demand functions. At this point it would seem that, to relate fixed points to equilibria of economies specified in terms of preferences and endowments of individual consumers, we would need to use some method, such as that of Geanakoplos (1984), that constructs an economy of $\mathrm{n}$ consumers for any arbitrary aggregate excess demand function. Thus, if we had an algorithm for computing equilibria of arbitrary economies specified in terms of preferences and endowments, using it to compute fixed points would be complicated: We would first use Uzawa's method to turn the mapping whose fixed points we wanted to compute into an excess demand function. We would then use Geanakoplos's method to turn this excess demand function into $\mathrm{n}$ pairs of utility functions and endowments vectors. Although the first step is trivial, the second is not. Rather than go into details, however, let us postpone discussion of this issue to Section 3, where we shall see that there is another connection between fixed points and equilibria of economies specified in terms of preferences and endowments. 


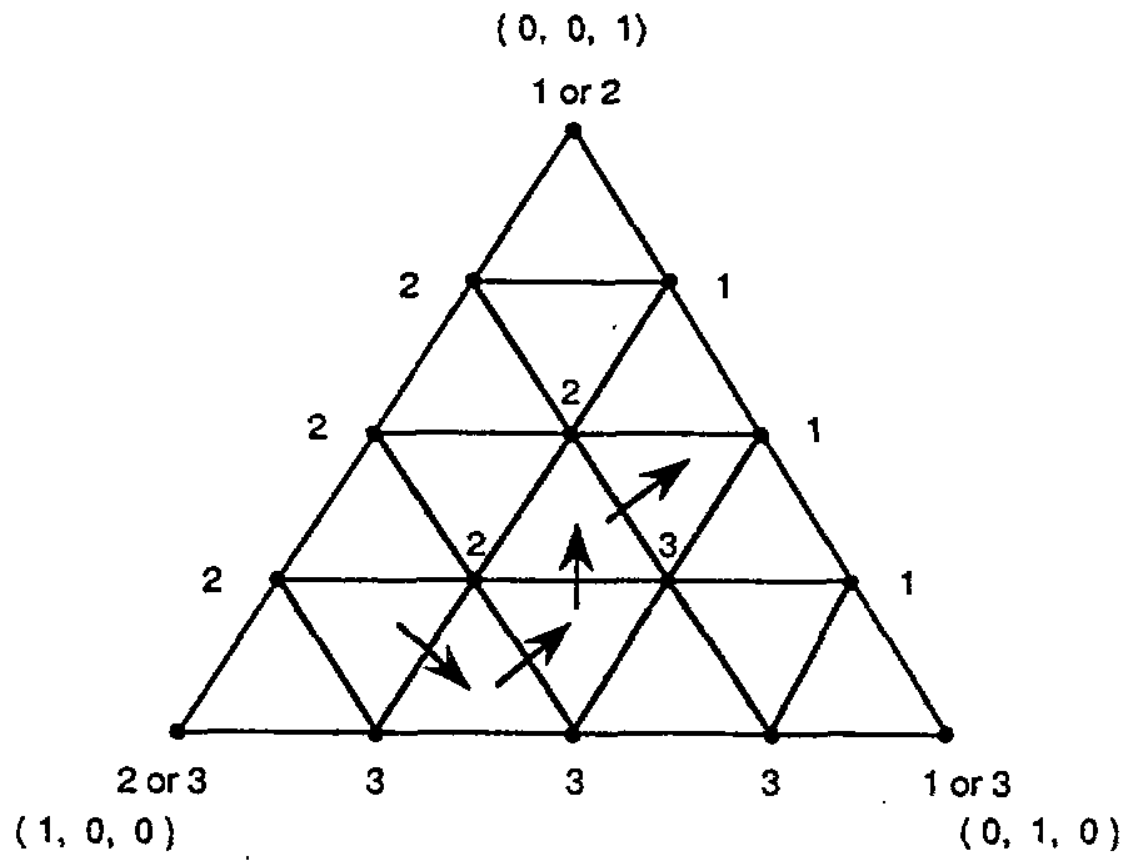

FIGURE 2.1 


\subsection{Scarf's Algorithm}

Uzawa's result says that any algorithm that is guaranteed to compute equilibria of arbitrary economies specified in terms of aggregate excess demand functions must be guaranteed to compute fixed points of arbitrary mapping of the simplex into itself. Scarf $(1967,1973,1982)$ has developed such an algorithm. Numerous researchers have further improved algorithms of this type, known as simplicial algorithms; see, for example, Merrill (1971), Eaves (1972), Kuhn and MacKinnon (1975), Todd (1976a), and van der Lann and Talman (1980). This type of algorithm can also be used to compute fixed points of set-valued correspondences.

In $\mathbf{R}^{\mathbf{n}}$ a $\mathrm{k}$ dimensional simplex is the convex hull of $\mathrm{k}+1$ points, called vertices, $v^{1}, \ldots$, $v^{k+1}$, that have the property that the $k$ vectors $v^{1}-v^{k+1}, \ldots, v^{k}-v^{k+1}$ are linearly independent. The price simplex $S$, for example, has vertices $e^{i}, i=1, \ldots, n$, where $e_{i}^{i}=1, e_{j}^{i}=0, j \neq 1$. A face of a simplex is a lower dimensional simplex whose vertices are vertices of the large simplex. In $\mathrm{R}^{3}$, for example, the point $\mathrm{e}^{1}$ is an 0 dimensional face of $S$ and the convex hull of $\mathrm{e}^{1}$ and $\mathrm{e}^{2}$ is a 1 dimensional face. A subdivision of $S$ divides $S$ into smaller simplices so that every point in $S$ is an element of some subsimplex and the intersection of any two subsimplices is either empty or a face of both.

Scarf's approach to computation of equilibria is based on a constructive proof of a version of Sperner's lemma: Assign to every vertex of a simplicial subdivision of $S$ a label, an integer from the set $\{1, \ldots, n\}$, with the property that a vertex $v$ on the boundary of $S$ receives a label $i$ for which $v_{i}=0$. Then there exists a subsimplex whose vertices have all of the labels $1, \ldots, n$.

Scarf's algorithm for finding this completely labeled subsimplex is to start in the corner of $\mathrm{S}$ where there is a subsimplex with boundary vertices with all of the labels $2, \ldots, n$. (See Figure 2.1.) If the additional vertex of this subsimplex has the label 1 , then the algorithm stops. Otherwise, it proceeds to a new subsimplex with all of the labels $2, \ldots, n$. The original subsimplex 
has two faces that have all of these labels. One of them includes the interior vertex. The algorithm moves to the unique other subsimplex that shares this face. If the additional vertex of this subsimpiex has the label 1 , the algorithm stops. Otherwise, it proceeds, moving to the unique subsimplex that shares the new face and has the labels $2, \ldots, \mathrm{n}$, and so on. The algorithm cannot try to exit through a boundary face. (Think of what labels the vertices of such a face must have.) Nor can it cycle. (To cycle there must be some subsimplex that is the first that the algorithm encounters for the second time; but the algorithm must have previously encountered both of the subsimplices that share the two faces of this subsimplex with the labels $2, \ldots, n$.) Since the subdivision consists of a finite number of subsimplices, the algorithm must terminate with a completely labeled subsimplex.

To see the connection of this aigorithm with Brouwer's theorem, we assign a vertex $\mathrm{v}$ with a label i for which $g_{i}(v) \geq v_{i}$. Since $e^{\prime} g(v)=e^{\prime} v=1$, there must be such an $i$. Notice that, since $\mathrm{g}_{\mathrm{i}}(\mathrm{v}) \geq 0, \mathrm{i}$ can be chosen such that the labeling convention on the boundary is satisfied. A completely labeled subsimplex has vertices $v^{1}, \ldots, v^{n}$ such that $g_{i}\left(v^{i}\right) \geq v_{i}^{i}, i=1, \ldots, n$. To prove Brouwer's theorem, we consider a sequence of subdivisions whose mesh, the maximum distance between vertices in the same subsimplex, approaches zero. Associate each subdivision with a point in a completely labeled subsimplex. Since $S$ is compact, this sequence of points has a convergent subsequence. Call the limit of this subsequence $x$. Since $g$ is continuous, we know $g_{i}(\hat{x}) \geq \hat{x}_{i}$, $i=1, \ldots, n$. Since $e^{\prime} g(x)=e^{\prime} x=1, g(x)=x$.

Scarf does not consider an infinite sequence of subdivisions, which is the nonconstructive aspect of this proof. Instead, he works with a subdivision with a small mesh. Any point in a completely labeled subsimplex serves as an approximate fixed point in the sense that $\|g(x)-x\|$ $<\epsilon$ where $\epsilon$ depends on the mesh and the modulus of continuity of $\mathrm{g}$. 


\subsection{The Global Newton Method}

An alternative algorithm for computing fixed points has been developed by Smale (1976), the global Newton method. It is based on Hirsch's (1963) proof of Brouwer's theorem. A similar method has been developed by Kellogg, Li, and Yorke (1976). Let $S$ now be the disk $\{x \in$ $\left.\mathbf{R}^{\mathrm{n}} \mid \mathbf{X}^{\prime} \mathbf{X} \leq 1\right\}$; like the simplex it is a nonempty, compact, convex set. Smale starts with an algorithm for computing fixed points of a continuously differentiable map $g: S \rightarrow S$ that has the property that $g(x)=0$ for every $x$ on the boundary of $S$, the sphere $\partial S=\left\{x \in R^{n} \mid x^{\prime} x=1\right\}$. Smale (1976) and Varian (1977) show how to extend this algorithm to situations where $\mathrm{g}$ is an arbitrary continuous map and $S$ is again the simplex.

Suppose that $S$ is the disk. If $\mathrm{g}: \mathrm{S} \rightarrow \mathrm{S}$ has no fixed point, we could define a map

$$
h(x)=\lambda(x)(x-g(x))
$$

where $\lambda(x)=\left((x-g(x))^{\prime}(x-g(x))\right)^{-1 / 2}$. This map would be a retraction of $S$ into its boundary: it would continuously map $S$ into $\partial S$ and be the identity on $\partial S$. Hirsch proves that no such map could exist, thereby proving Brouwer's Theorem. Smale proposes starting with a regular value of $x-g(x)$, a point $\bar{x} \in \partial S$ such that $I-\operatorname{Dg}(\bar{x})$ is nonsingular. Sard's theorem says that the set of regular values has full measure and, in particular, that there exists such a point $\bar{x}$. The algorithm then follows the solution to

$$
\lambda(x(t))(x(t)-g(x(t)))=\bar{x}
$$

Since the path $x(t)$ cannot return to any other boundary point, and since it cannot return to $\bar{x}$ because it is a regular value, it must terminate at a fixed point.

Differentiating the above equation with respect to $t$, we obtain

$$
\lambda(\mathrm{x})(\mathrm{I}-\mathrm{Dg}(\mathrm{x})) \dot{\mathrm{x}}+\dot{\lambda}(\mathrm{x}-\mathrm{g}(\mathrm{x}))=0 \text {. }
$$




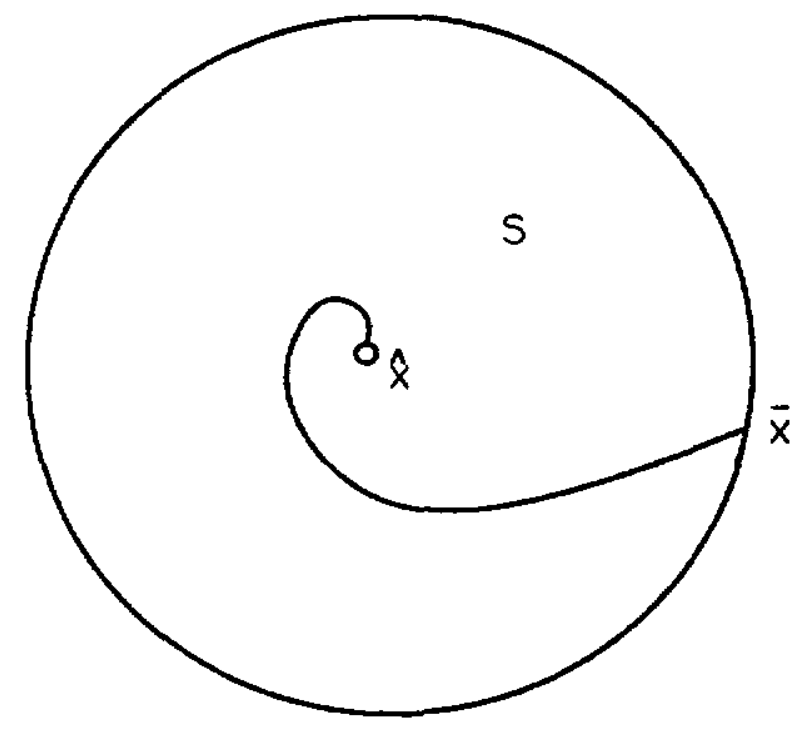

FIGURE 2.2 
Smale shows that $x(t)$ can be chosen as the solution to the differential equation

$$
(I-D g(x)) \dot{x}=\mu(x)(g(x)-x)
$$

where $\mu(x)$ has the same sign as $\operatorname{det}[\mathrm{I}-\mathrm{Dg}(\mathrm{x})]$ and is scaled so that $\dot{x}$ has constant velocity. Except for the factor $\mu$, this is a continuous version of Newton's method for solving $x-g(x)=0$ :

$$
x_{t+1}=x_{t}-\left(I-D g\left(x_{t}\right)\right)^{-1}\left(x_{t}-g\left(x_{t}\right)\right) .
$$

\subsection{Regularity and the Index Theorem}

Merely establishing the existence of equilibria and developing methods for computing them leaves important questions unanswered: Are equilibria unique? If not, are they locally unique? Do they vary continuously with the parameters of the economy? In recent years, economists have used the tools of differential topology to investigate these questions. Debreu (1970) has investigated the questions of local uniqueness and continuity with continuously differentiable excess demand functions. See Dierker (1982) and Mas-Colell (1985) for surveys of this and subsequent work. Analogous results to those derived in the differentiable framework can be obtained in a piecewise-linear framework applicable to Scarf's approach to computing equilibria. See, for example, Eaves and Scarf (1976) and Eaves (1976).

Debreu (1970) defines a regular economy to be one that satisfies conditions sufficient for there to be a finite number of equilibria. Dierker and Dierker (1972) simplify these conditions to the requirement that the Jacobian matrix of excess demands $p$ with the first row and column deleted, the $(n-1) \times(n-1)$ matrix $\bar{J}$, is nonsingular at every equilibrium. The first row is deleted because of Walras's law, the first column because of homogeneity. We are left with a square matrix because, as Walras (1874, Lesson 12) pointed out, the number of equations equals the number of unknowns in the equilibrium conditions. The inverse function theorem implies that every equilibrium 
of a regular economy is locally unique. Since the set $S$ is compact and the equilibrium conditions involve continuous functions, this implies that a regular economy has a finite number of equilibria.

Let us rewrite the equilibrium conditions as $f(p, b)=0$ where $b \in B$ and $B$ is a topological space of parameters. If $\mathrm{f}$ and its partial derivatives with respect to $\mathrm{p}$ are continuous in both $\mathrm{p}$ and b, then the implicit function theorem implies that equilibria vary continuously at regular economies. Furthermore, in the case where $B$ is the set of possible endowment vectors $w^{i}$, Debreu uses Sard's theorem to prove that, for every $b$ in an open set of full measure in $B, f(\cdot, b)$ is a regular economy. When $B$ is the function space of excess demand functions with the uniform $C^{1}$ topology, an open dense set of B consists of regular economies. Consequently, if we are willing to restrict attention to continuously differentiable excess demand functions, a restriction that Debreu (1972) and Mas-Colell (1974) have shown is fairly innocuous, almost all economies, in one of two alternative, precise mathematical senses, are regular.

Dierker (1972) has noticed that a fixed point index theorem could be used to count the number of equilibria of a regular economy. Let us define the fixed point index of a regular equilibrium $p$ as $\operatorname{sgn}(\operatorname{det}[\mathrm{I}-\mathrm{Dg}(\hat{\mathrm{p}})])$ whenever this expression is nonzero. Dierker shows that the index can also be written as $\operatorname{sgn}(\operatorname{det}[-\overline{\mathrm{J}}])$. The index theorem says that $\Sigma$ index $(\hat{Q})=+1$ where the sum is over equilibria of a regular economy. This result is depicted in Figure 2.3 where $n=2$, $\mathrm{p}_{1}=1-\mathrm{p}_{2}$, and $\mathrm{g}_{1}\left(\mathrm{p}_{1}, \mathrm{p}_{2}\right)=1-\mathrm{g}_{2}\left(\mathrm{p}_{1}, \mathrm{p}_{2}\right)$. Here index $(\hat{\rho})=\operatorname{sgn}\left(1-\partial \mathrm{g}_{2} / \partial \mathrm{p}_{2}\right)$ and a regular economy is one where the graph of $\mathrm{g}$ does not become tangent to the diagonal.

Mas-Colell (1977) shows that any compact subset of $\mathrm{S}$ can be the equilibrium of some economy f. If we restrict ourselves to regular economies and $n \geq 3$, then the only restrictions placed on the number of equilibria are those given by the index theorem. (If $n=2$, an equilibrium with index -1 must lie between two with index +1 .) This implies that the number of equilibria is odd and that there is a unique equilibrium if and only if index $(\hat{p})=+1$ at every equilibrium. 
$Z$ 
It is easy to see that there are an odd number of solutions to Scarf's algorithm and to Smale's global Newton method: To see this in the case of Scarf's algorithm, let us argue that there are an odd number of completely labeled subsimplices. The path followed from the corner missing the label 1 leads to a unique subsimplex. Suppose there is an additional completely labeled subsimplex. Then it shares the face with labels $2, \ldots, \mathrm{n}$ with a unique other subsimplex. Restart Scarf's algorithm at this subsimplex. Either the additional vertex to this subsimplex has the label 1 , in which case it is completely labeled, or it does not, in which case it has another face with all of the labels $2, \ldots, n$. Move to the unique other subsimplex that shares this face and continue as before. This algorithm cannot encounter any subsimplex in the path from the corner to the original subsimplex. (To do so there must be some subsimplex in the path that is the first that it encounters; but it must have previously encountered both of the subsimplices that share the two faces of this subsimplex with the labels $2, \ldots, n$.) The algorithm must therefore terminate in yet another completely labeled subsimplex. Consequently, all completely labeled subsimplices, except the original one located by the algorithm starting in the corner, come in pairs. There is a definition of index of a completely labeled subsimplex that agrees with that of a fixed point $\dot{p}$ in the case where the mesh of the subdivision is sufficiently small and $\mathrm{f}$ is regular (see Eaves and Scarf 1976 and Todd 1976b). The original subsimplex located by the algorithm starting in the corner has index +1 . All other completely labeled subsimplices come in pairs as described above, one with index +1 and one with index -1 .

Likewise, it can be shown that the global Newton method has an odd number of solutions. Starting at $\bar{x}$ on the boundary the algorithm locates one, which has index +1 . All other solutions are matched up in pairs, one with index +1 and one with index -1 . Indeed, it is a general fearure of these and related algorithms that, unless they are restarted at a fixed point different from the one originally computed by the algorithm, they always lead to fixed points with index +1 . This, combined with Mas-Colell's (1977) result about the arbitrariness of the number of fixed points, 


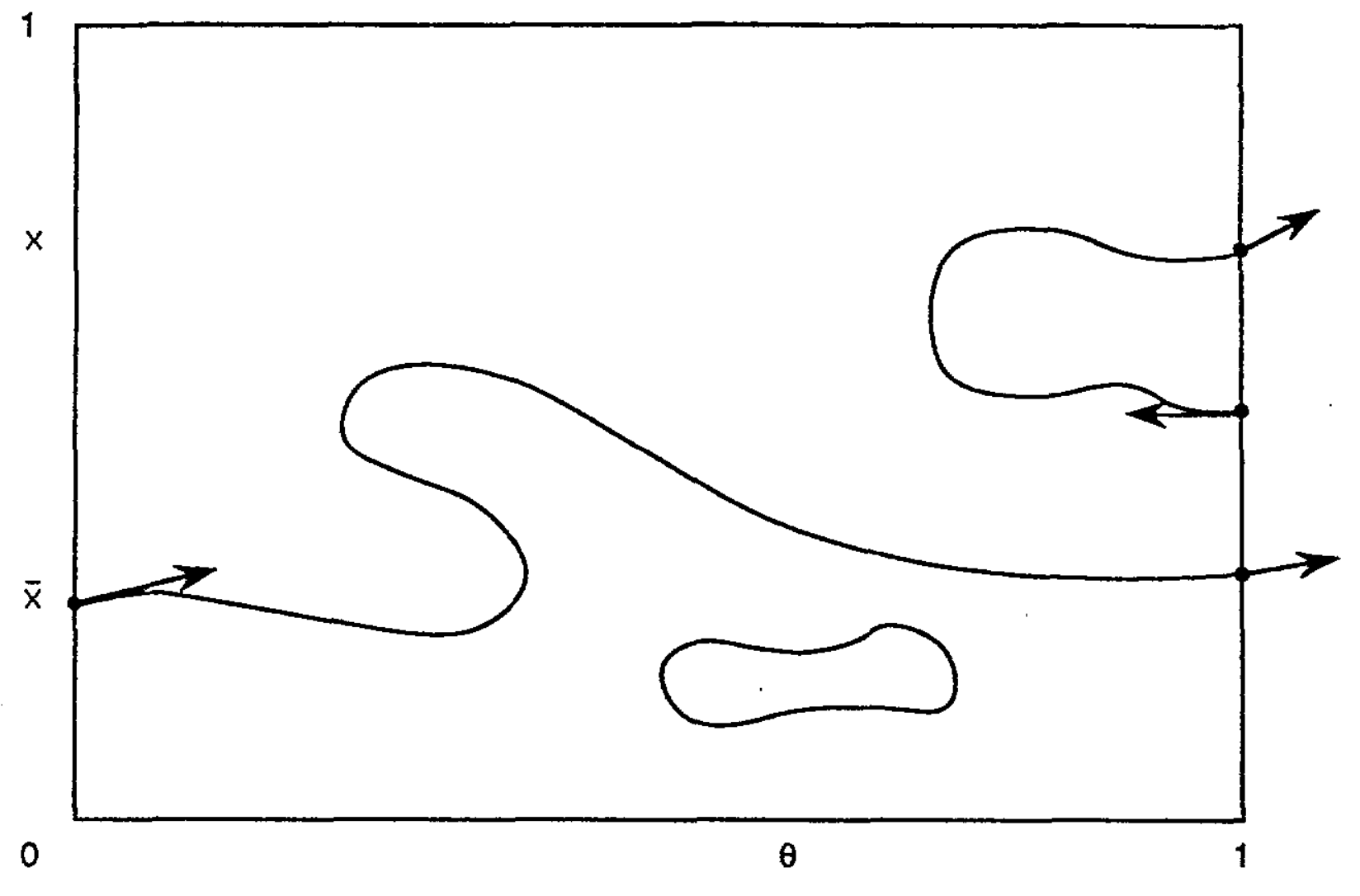

FIGURE 2.4 
suggests that, unless for some reason we know that index $(\hat{p})=+1$ at every fixed point, there can be no method except for an exhaustive search that locates all fixed points. There is an important possible exception to this remark involving the all-solutions algorithm of Drexler (1978) and Garcia and Zangwill $(1979,1981)$. This method, which depends on being able to globally bound $\mathrm{g}$ using complex polynomial functions, is further discussed in the next section.

\subsection{Path Following Methods}

Much recent work on the computation of fixed points has been based on the idea of path following. The idea is to follow the path of solutions to $H(x, \theta)=0$ where $H: S \times[0,1] \rightarrow R^{n}$ is chosen so that $H(x, 0)=0$ is trivial to solve and so that $H(x, 1)=x-g(x)$, which means a solution to $H(x, 1)=0$ is a fixed point. The function $H$ is called a homotopy. (See Garcia and Zangwill 1981 for a survey and references.)

Suppose that $\mathrm{g}: \mathrm{S} \rightarrow \mathrm{S}$ is twice continuously differentiable. Define

$$
\mathrm{H}(\mathrm{x}, \theta)=\mathrm{x}-(1-\theta) \overline{\mathrm{x}}-\theta \mathrm{g}(\mathrm{x})
$$

where $\bar{x}$ is an interior point of $S$. Notice that, for any $1>\theta \geq 0$ and $x \in S,(1-\theta) \bar{x}+\theta g(x)$ is also interior to $S$. We start at the trivial solution $H(\bar{x}, 0)=0$ and follow the solution path until we reach the boundary where $\theta=1$ and $H(x, 1)=x-g(x)=0$. We require that 0 be a regular value of $\mathrm{H}(\mathrm{x}, \theta)$ in the sense that the $\mathrm{n} \times(\mathrm{n}+1)$ matrix $\mathrm{DH}(\mathrm{x}, \theta)$ has tank $n$ whenever $\mathrm{H}(\mathrm{x}, \theta)=0$. Sard's theorem says that we can always choose $\bar{x}$ so that this condition is satisfied and, indeed, that it is satisfied for almost all $\overline{\mathrm{x}}$. (It is here that second differentiability is important.) The implicit function theorem then implies that solutions to $\mathrm{H}(\mathrm{x}, \theta)=0$ form a compact one dimensional manifold with boundary, a finite number of paths and loops, and that the boundary points of this manifold are also boundary points of $S \times[0,1]$. By construction $H$ is such that $(\bar{x}, 0)$ is the only possible boundary solution except for points where $\theta=1$, where solutions are fixed points of $\mathrm{g}$. (See Figure 2.4.) 
Although the path that starts at $(\bar{x}, 0)$ cannot return to the boundary where $\theta=0$, it need not be monotonic in $\theta$. Consequently, we do not want to think of the path in terms of $x$ as a function of $\theta$. Rather, let us write $y(t)=(x(t), \theta(t))$. Differentiating $H(y(t)) \equiv 0$ with respect to $t$, we obtain $\mathrm{DH}(\mathrm{y}) \dot{\mathrm{y}}=0$.

This is a system of $\mathrm{n}$ linear equations in $\mathrm{n}+1$ unknowns that has an infinite number of solutions. One is

$$
\dot{y}_{i}=(-1)^{n-i+1} \operatorname{det} \operatorname{DH}(y)_{-i}
$$

Here $\mathrm{DH}(\mathrm{y})_{-\mathrm{i}}$ is the $\mathrm{n} \times \mathrm{n}$ matrix formed by deleting column $\mathrm{i}$ from $\mathrm{DH}(\mathrm{y})$. That 0 is a regular value of $\mathrm{H}$ implies that at every point $y$ along the path some matrix $\mathrm{DH}(\mathrm{y})_{-\mathrm{i}}$ is nonsingular. To see that the above differential equation does indeed follow the solution path to $H(y)=0$, we suppose that $\left.\mathrm{DH}_{(\mathrm{y}}\right)_{-1}$ is nonsingular and rewrite $\mathrm{DHy}=0$ as

$$
\sum_{i=2}^{n+1} D_{i} H \dot{y}_{i}=-D_{1} H \dot{y}_{1}
$$

where $\mathrm{D}_{\mathrm{i}} \mathrm{H}$ is column $\mathrm{i}$ of $\mathrm{DH}$. We choose $\dot{\mathrm{y}}_{1}=(-1)^{\mathrm{n}}$ det $\mathrm{DH}_{-1}$ and solve for $\dot{\mathrm{y}}_{2}, \ldots, \dot{y}_{\mathrm{n}+1}$ using Cramer's rule:

$$
\begin{aligned}
\dot{y} & =\operatorname{det}\left[\mathrm{D}_{2} \mathrm{H} \cdots \mathrm{D}_{\mathrm{i}-1} \mathrm{H} \quad(-1)^{\mathrm{n}+1}\left(\operatorname{det} \mathrm{DH} \mathrm{H}_{-1}\right) \mathrm{D}_{1} \mathrm{H} \quad \mathrm{D}_{\mathrm{i}+1} \mathrm{H} \cdots \mathrm{D}_{\mathrm{n}+1} \mathrm{H}\right] / \operatorname{det} \mathrm{DH}_{-1} \\
& =(-1)^{\mathrm{n}-\mathrm{i}+1} \operatorname{det} \mathrm{DH}_{-\mathrm{i}^{*}}
\end{aligned}
$$

As with the global Newton method, we have reduced the problem of computing fixed points to that of solving a system of ordinary differential equations. To solve such equations, we can use a variety of methods, such as the Runge-Kutta method or the Bulirsch-Stoer method (see, for example, Gear 1971 and Stoer and Bulirsch 1980, Chapter 7). The homotopy approach can also be applied to piecewise-linear problems (see, for example, Merrill 1971, Eaves 1972, Kuhn and MacKinnon 1975, Eaves 1976, and Eaves and Scarf 1976). 
The homotopy approach yields a very simple proof of the index theorem. Notice that at $(\bar{x}, 0)$

$$
\grave{\theta}=\operatorname{det}\left[\mathrm{DH}(\overline{\mathrm{x}}, 0)_{-(\mathrm{n}+1)}\right]=\operatorname{det} \mathrm{I}=1>0 .
$$

Following the path of solutions to $\mathrm{H}(\mathrm{x}(\mathrm{t}), \theta(\mathrm{t}))=0, \dot{\theta}$ may change signs, but when $\theta=1$

$$
\dot{\theta}=\operatorname{det}\left[\mathrm{DH}(\mathrm{x}, 1)_{-(\mathrm{a}+1)}\right]=\operatorname{det}[\mathrm{I}-\mathrm{Dg}(\mathrm{x})]
$$

must be nonnegative. (Take another look at Figure 2.4.) If 0 is a regular value of $x-g(x)$, if the economy is regular, then $\operatorname{det}[I-\operatorname{Dg}(x)]>0$. Other fixed points come in pairs, with each one the endpoint of a path that starts and ends on the boundary where $\theta=1$. At one endpoint $\dot{\theta} \leq 0$ and at the other $\dot{\theta} \geq 0$. In the regular case we define index $(R)=\operatorname{sgn}(\operatorname{det}[I-D g(R)])$. Summing over all fixed points, all solutions to $H(x, 1)=0$, yields +1 . This proof of the index theorem is easily extended to maps that are continuously differentiable only of frrst, rather than second, order (see Garcia and Zangwill 1981, Chapter 22).

A fascinating possibility presented by the path following idea is that of being able to compute all of the fixed points of a function $\mathrm{g}: \mathrm{S} \rightarrow \mathrm{S}$. The all-solutions algorithm of Drexler (1978) and Garcia and Zangwill (1979) is easiest understood in terms of computing zeros of polynomials. We first approximate $g(x)-x$ by a finite order polynomial $f: S \rightarrow R^{n}$ and then extend $f$ to a function $f: R^{n} \rightarrow R^{n}$. Weierstrass's approximation theorem says that we can choose $f$ to approximate $g(x)-x$ arbitrary closely on $S$ (see, for example, Lang 1983, pp. 49-53). We then convert $f$ into a complex function by allowing both its domain and range to be $\mathrm{C}^{\mathrm{n}}$, the space of complex $\mathrm{n}$ vectors. We can expand the vector $z \in C^{n}$ into a vector $z^{*} \in R^{2 n}$ by writing $z=\left(z_{1}^{*}+z_{2}^{*} i, \ldots, z_{2 n-1}^{*}+z_{2 n}^{*} i\right)$. Consequently, we can expand $f$ into $f^{*}: R^{2 n} \rightarrow R^{2 n}$ by writing $f(z)=\left(f_{1}^{*}\left(z^{*}\right)+f_{2}^{*}\left(z^{*}\right) i, \ldots\right.$, $\left.\mathrm{f}_{2 \mathrm{n}-1}^{*}\left(\mathrm{z}^{*}\right)+\mathrm{f}_{2 \mathrm{n}}^{*}\left(\mathrm{z}^{*}\right) \mathrm{i}\right)$. We now discuss a method that can compute all the zeros of $\mathrm{f}^{*}$. Notice that 
not all of the zeros of $\mathrm{f}^{*}$ are approximate fixed points of $\mathrm{g}$ : some may be complex and some may lie outside of S.

Letting $m_{j}$ be the highest order of the polynomial $f_{j}(z)$, we consider the homotopy $H: C^{n} \times$ $[0,1] \rightarrow C^{n}$ defined by the rule

$$
H_{j}(z, \theta)=(1-\theta)\left(z_{j}^{\left(m_{j}+1\right)}-1\right)+\theta f_{j}(z), \quad j=1, \ldots, n .
$$

At $\theta=1$ solutions to $\mathrm{H}(\mathrm{z}, \theta)=0$ are zeros of $\mathrm{f}$. At $\theta=0, \mathrm{H}_{\mathrm{j}}(z, \theta)=0$ has $\mathrm{m}_{\mathrm{j}}+1$ solutions

$$
z_{j}=\cos \left(2 \pi a /\left(m_{j}+1\right)\right)+i \sin \left(2 \pi a /\left(m_{j}+1\right)\right), \quad a=0,1, \ldots, m_{j}
$$

Consequently, there are $\Pi_{j=1}^{\mathrm{n}}\left(\mathrm{m}_{\mathrm{j}}+1\right)$ solutions to $H(z, 0)=0$. We can expand $H$ into $H^{*}: R^{2 n} \times[0,1] \rightarrow R^{2 n}$. The crucial insight involved in the all-solutions algorithm is that any solution path to $\mathrm{H}^{*}\left(\mathrm{z}^{*}, \theta\right)=0$ is monotonic in $\theta$,

$$
\dot{\theta}=\operatorname{det}\left[\mathrm{DH}^{*}\left(\mathbf{z}^{*}, \theta\right)-(2 \mathbf{n}+1)\right] \geq 0 \text {. }
$$

The proof is simple: $\mathrm{DH}^{*}\left(\mathrm{z}^{*}, \theta\right)_{-(2 \mathrm{n}+1)}$ consists of $2 \times 2$ blocks of the form

$$
\left[\begin{array}{ll}
\partial \mathrm{H}_{\mathrm{i}}^{\mathrm{r}} / \partial z_{j}^{\mathrm{r}} & \partial \mathrm{H}_{\mathrm{i}}^{\mathrm{T}} / \partial z_{j}^{\mathrm{i}} \\
\partial \mathrm{H}_{\mathrm{i}}^{\mathrm{i}} / \partial z_{j}^{\mathrm{r}} & \partial \mathrm{H}_{\mathrm{i}}^{\mathrm{i}} / \partial \mathrm{z}_{\mathrm{j}}^{\mathrm{i}}
\end{array}\right] \text {. }
$$

Here $z_{j}^{r}=z_{2 j-1}^{*}$ is the real part of $z_{j}, z_{j}^{i}=z_{2 j}^{*}$ is the imaginary part, and $H_{i}^{r}$ and $H_{i}^{i}$ are the real and imaginary parts of $H_{\mathrm{i}}$. The Cauchy-Riemann equations, which follow easily from the chain rule, say that

$$
\begin{aligned}
& \frac{\partial \mathrm{H}_{i}^{r}}{\partial z_{j}^{r}}=\frac{\partial H_{i}^{i}}{\partial z_{j}^{i}} \\
& \frac{\partial H_{i}^{r}}{\partial z_{j}^{i}}=-\frac{\partial H_{i}^{i}}{\partial z_{j}^{r}}
\end{aligned}
$$


Consequently, the $2 \times 2$ blocks that make up $\mathrm{DH}^{*}\left(\mathrm{z}^{*}, \theta\right)-(2 \mathrm{n}+\mathrm{l})$ all have the special form

$$
\left[\begin{array}{rr}
a_{i j} & b_{i j} \\
-b_{i j} & a_{i j}
\end{array}\right] \text {. }
$$

These matrices have important properties: their special form is preserved when such a matrix is multiplied by a scalar or inverted; it is also preserved when two such matrices are added or multiplied together. Consequently, performing Gaussian elimination on these $2 \times 2$ blocks, we can reduce the $2 \mathrm{n} \times 2 \mathrm{n}$ matrix $\mathrm{DH}^{*}\left(\mathrm{z}^{*}, \theta\right)_{-(2 \mathrm{n}+1)}$ to a lower block triangular matrix with $\mathrm{n}$ such $2 \times 2$ blocks on the diagonal. The determinant is the product of the determinants of these blocks, each of which is nonnegative.

Since $\theta$ is monotonic along any path, there can be no paths that both start and end at $\theta=0$ or at $\theta=1$. To guarantee that every solution at $\theta=1$ is the endpoint of a path that starts with $\theta=0$, we need to rule out paths diverging to infinity for $0 \leq \theta<1$. It is here that the polynomials $\left(z_{j}^{\left(m_{j}+1\right)}-1\right)$ play their role: Suppose that $\|z\| \rightarrow \infty$. Then, for at least one $i$, $f_{j}(z) /\left(z_{j}^{\left(m_{j}+1\right)}-1\right) \rightarrow 0$, which implies that $H_{j}(z, \theta) /\left(z_{j}^{\left(m_{j}+1\right)}-1\right) \rightarrow(1-\theta)$. Consequently, $H_{j}(z, \theta)=0$ cannot hold for any path along which $\|x\| \rightarrow \infty$ and $0 \leq \theta<1$. Following each of the paths that starts at $\theta=0$ either leads to a zero of $\mathrm{f}$ or diverges to infinity at $\theta=1$. No path can start at $\theta=1$ and diverge to infinity going backwards, however, so this method necessarily locates all of the zeros of f. (See Garcia and Zangwill 1981, Chapter 18 for further discussion.)

This method can be applied to functions other than polynomials. What we need is a function $f: R^{n} \rightarrow R^{n}$ than can be extended to $C^{\mathfrak{n}}$ and polynomials $\left(z_{i}^{q_{i}}-1\right)$ such that some $f_{i}(z) /\left(z_{i}^{q_{i}}-1\right) \rightarrow 0$ as $\|z\| \rightarrow \infty$. The all-solutions algorithm is obviously a promising direction for future research. 


\subsection{Multiplicity of Equilibria}

By constructing an example of an economy with an equilibrium with index -1 , we can easily construct an example of multiplicity of equilibria.

Example 2.1. Consider a static exchange economy with two consumers and two goods. Consumer $i, i=1,2$, has a utility function of the form

$$
u_{i}\left(x_{1}, x_{2}\right)=\sum_{j=1}^{2} a_{j}^{i}\left(x_{j}^{b_{i}}-1\right) / b_{i}
$$

where $a_{j}^{i} \geq 0$ and $b_{i}<1$. This is, of course, the familiar constant-elasticity-of-substitution utility function with elasticity of substitution $\eta_{i}=1 /\left(1-b_{i}\right)$. Given an endowment vector $\left(w_{1}^{i}, w_{2}^{i}\right)$, consumer $\mathrm{i}$ maximizes this utility subject to his budget constraint. His demand functions are

$$
x_{j}^{i}\left(p_{1}, p_{2}\right)=\frac{\gamma_{j}^{i} \sum_{k=1}^{2} p_{k} w_{k}^{i}}{p_{j}^{\eta_{i}} \sum_{k=1}^{2} \gamma_{k}^{i} p_{k}^{1-\eta_{i}}}, i=1,2 ; j=1,2
$$

Here $\gamma_{j}^{i}=\left(a_{j}^{i}\right)^{\eta_{i}}$. The two consumers have the following (symmetric) parameters:

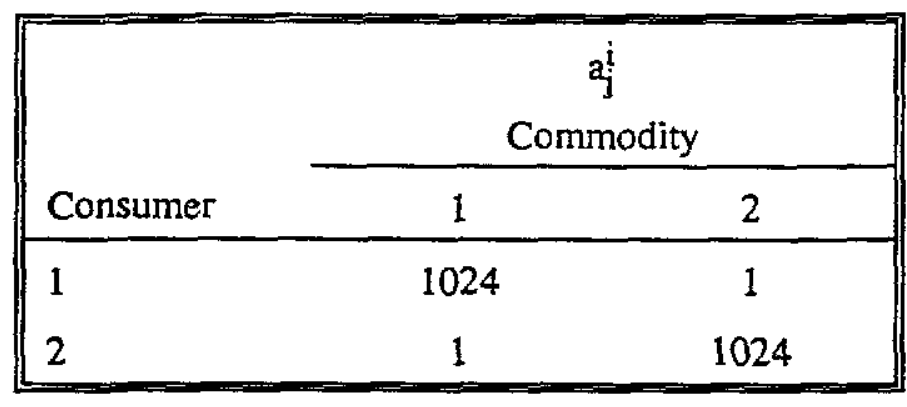

$$
b_{1}=b_{2}=-4
$$




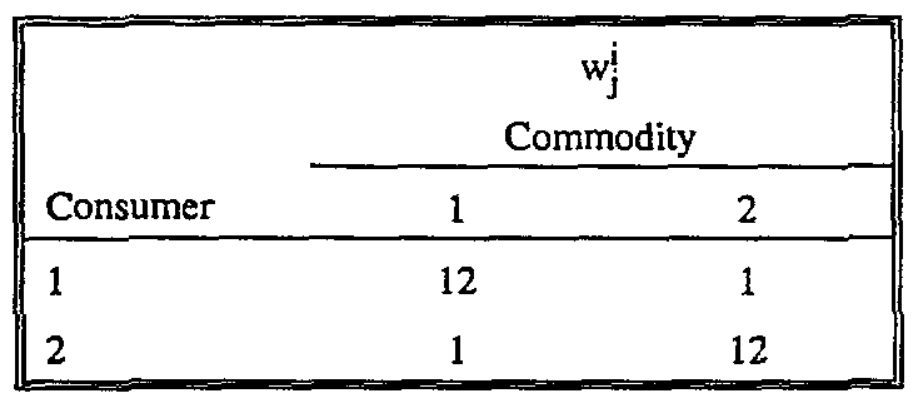

Of course $\eta_{1}=\eta_{2}=1 / 5, \gamma_{1}^{1}=\gamma_{2}^{2}=4$, and $\gamma_{2}^{1}=\gamma_{1}^{2}=1$.

This economy has three equilibria, which are listed below:

\begin{tabular}{|c|c|c|c|}
\hline \multirow[b]{2}{*}{ Consumer } & \multicolumn{2}{|c|}{$\begin{array}{c}\text { EQUILIBRIUM } 1 \\
\mathrm{p}^{1}=(0.5000,0.5000) \\
\text { Commodity } \\
\end{array}$} & \multirow[b]{2}{*}{$\mathrm{u}_{\mathrm{i}}$} \\
\hline & 1 & 2 & \\
\hline 1 & 10.400 & 2.600 & -0.02735 \\
\hline 2 & 2.600 & 10.400 & -0.02735 \\
\hline
\end{tabular}
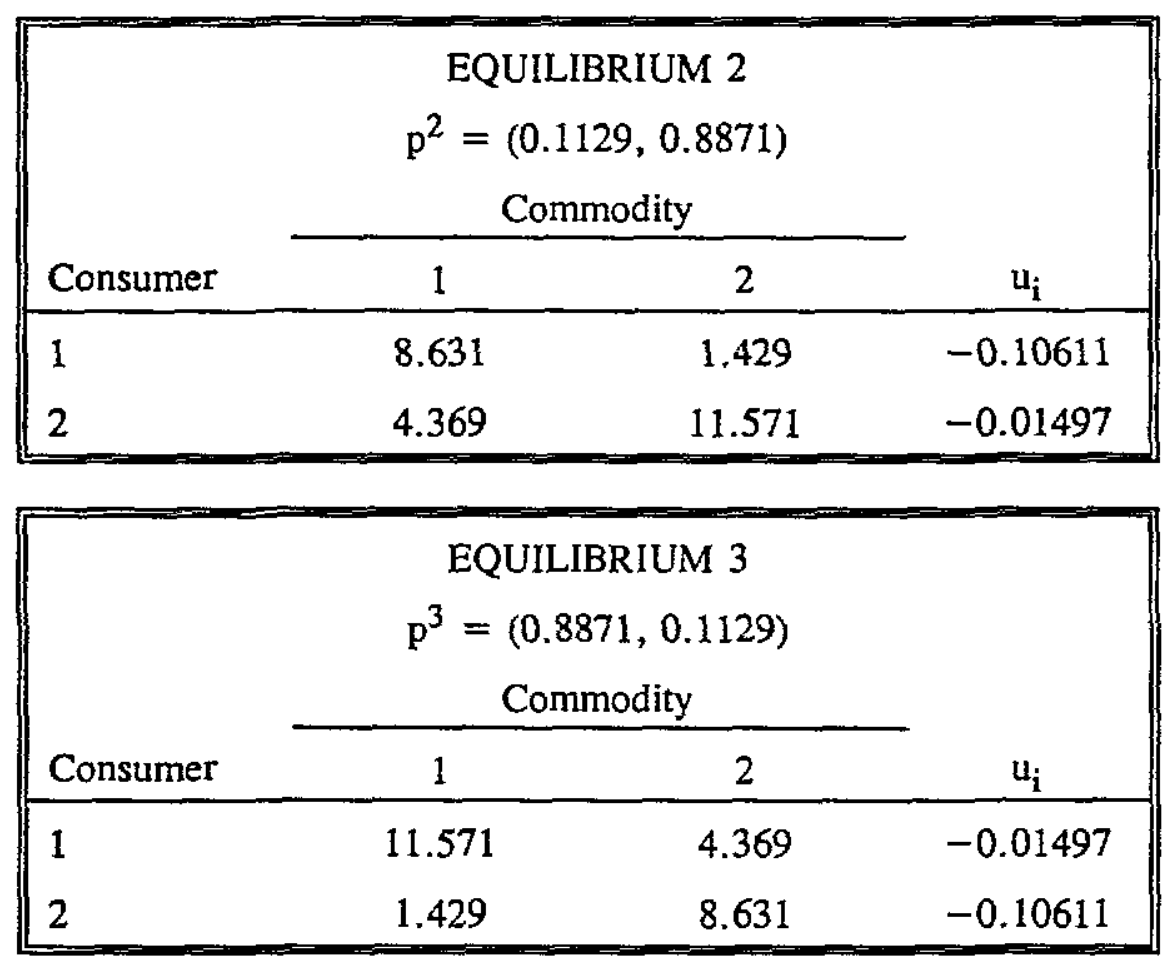
This example has been constructed by making $\mathrm{p}^{1}=(0.5,0.5)$ an equilibrium with index -1 ,

$$
\operatorname{Df}\left(\mathrm{p}^{1}\right)=\left[\begin{array}{rr}
3.2 & -3.2 \\
-3.2 & 3.2
\end{array}\right],
$$

index $\left(p^{1}\right)=\operatorname{sgn}(-3.2)=-1$.

Remark. A similar example has been constructed in an Edgeworth box diagram by Shapley and Shubik (1977).

Two assumptions have played significant roles in discussions of uniqueness of equilibria since the time of Wald (1936). They are gross substitutability and the weak axiom of revealed preference. Gross substitutability says that, if $p \geq q$ and $p_{i}=q_{i}$ for some $i$, then $f_{i}(p) \geq f_{i}(q)$ and, if $f(p)=$ $f(q)$, then $p=q$. (This actually combines the two conditions often known as weak gross substitutability and indecomposability.) The weak axiom of revealed preference says that if $p^{\prime} f(q) \leq 0$ and $q^{\prime} f(p) \leq 0$, then $f(q)=f(p)$.

The argument that gross substitutability implies uniqueness is easy: Suppose that there are two vectors $p, q$, such that $f(p)=f(q) \leq 0$. It must be the case that $p, q>0$. Otherwise, for example, $p_{i}=0$ and $2 p \geq p$ would imply $f_{i}(2 p)>f_{i}(p)$, which would contradict homogeneity. Let $\gamma=\max q_{j} / p_{j}$. Then $\gamma p$ satisfies $\gamma p \geq q, \gamma p_{i}=q_{i}$ some $i$. Consequently, $f(\gamma p)=f(p)=f(q)=0$ implies $\gamma \mathrm{p}=\mathrm{q}$. It is also easy to show that, when $\mathrm{f}$ is continuously differentiable, gross substitutability implies that index $(\hat{\beta})=+1$, since $\partial f_{i}(\hat{\phi}) \geq 0, i \neq j$ implies, in general, that $-\bar{J}$ is a P matrix, a matrix with all of its leading minors positive (see Hahn 1958 and Kehoe 1985b).

The weak axiom implies that the set of equilibria is convex. If $f$ is regular, this implies that it has a unique equilibrium. Suppose that there are two vectors $p$, $q$ such that $f(p)=f(q) \leq 0$. Then $\mathrm{p}(\theta)=\theta \mathrm{p}+(1-\theta) \mathrm{q}, 0 \leq \theta \leq 1$, satisfies $\mathrm{p}(\theta)^{\prime} \mathrm{f}(\mathrm{p}) \leq 0$ and $\mathrm{p}(\theta)^{\prime} \mathrm{f}(\mathrm{q}) \leq 0$. Walras's law implies that $(\theta p+(1-\theta) q)^{\prime} f(p(\theta))=0$. Consequently, it cannot be the case that $p^{\prime} f(p(\theta))>0$, 
otherwise $\mathrm{q}^{\prime} f(\mathrm{p}(\theta))<0$, which contradicts the weak axiom. Consequently, $\mathrm{p}^{\prime} \mathrm{f}(\mathrm{p}(\theta)) \leq 0$, which implies $f(p(\theta))=f(p) \leq 0$. In the case where $f$ is differentiable, the weak axiom implies that $D f(p)$ satisfies $x^{\prime} \operatorname{Df}(p) x \leq 0$ for all $x$ such that $x^{\prime} f(p)=0$ (see Kihlstrom, Mas-Colell, and Sonnenschein 1976; Kehoe 1985c; and Freixas and Mas-Colell 1987). This implies that $\operatorname{det}[\overline{\mathrm{J}}]>0$ if $\mathrm{p}$ is a regular equilibrium.

One problem with the weak axiom is that, in contrast to gross substitutability, it does not aggregate: the two functions in Example 2.1, for example, satisfy the weak axiom because they come from utility maximization; their sum obviously does not. Mas-Colell (1989) discusses a condition called monotonicity that both implies the weak axiom and aggregates: $f$ is monotone with respect to the normalizing vector $a \in R_{+}^{n}$ if $p^{\prime} a=q^{\prime} a=1$ and $p \neq q$ imply $(p-q)^{\prime}(f(p)-$ $\mathrm{f}(\mathrm{q}))<0$. Unfortunately, monotonicity is not implied by utility maximization. Mas-Colell (1989), however, presents sufficient conditions on utility functions and endowments for monotonicity to hold.

\subsection{Other Computational Methods}

Despite not being guaranteed to converge for arbitrary economies, methods for computing equilibria other than fixed point algorithms are popular in practice. Let us briefly consider three such methods, tâtonnement, a nonlinear Gauss-Seidel method, and Newton's method.

Samuelson (1941) has formalized Walras's (1874) concept of tonnement, or groping to equilibrium, as the system of differentiable equations

$$
\dot{p}=f(p) \text {. }
$$

Notice that $\|p\|$ stays constant under this adjustment process:

$$
\mathrm{d} / \mathrm{dt}\left(\mathrm{p}^{\prime} \mathrm{p}\right)=2 \mathrm{p}^{\prime} \dot{\mathrm{p}}=2 \mathrm{p}^{\prime} \mathrm{f}(\mathrm{p})=0
$$

In other words, if $\|p(0)\|=1$, then $\|p(t)\|=1$; the path followed by tatonnement always remains on the intersection of the sphere and the positive orthant. 
This process converges to the set of equilibria if $f$ satisfies the weak axiom, as shown by Arrow and Hurwicz (1958), who use the Liapunov function $L(p)=(1 / 2)(p-p)^{\prime}(p-p)$. Notice that $L(p)>0$ unless $p=p$ and that

$$
\dot{\mathrm{L}}(\mathrm{p})=(\mathrm{p}-\hat{\mathrm{p}})^{\prime} \dot{\mathrm{p}}=-\hat{\mathrm{p}} \mathrm{f}(\mathrm{p}) \text {. }
$$

Unless $p$ is an equilibrium, $f(\hat{p})=0$ and the weak axiom imply that $L(p)<0$. Arrow, Block, and Hurwicz (1959) further argue that gross substitutability implies that the weak axiom holds in comparisons with an equilibrium vector of an exchange model; that $f(\hat{p})=0$ implies $\hat{p}^{\prime} f(p)>0$ unless $f(p)=f(p)$. Consequently, tâtonnement is also globally asymptotically stable if $f$ satisfies gross substitutability.

If, however, $f$ does not satisfy the weak axiom or gross substitutability, the tatonnement process may not converge to an equilibrium. In fact, Scarf (1960) constructs a simple example with unique equilibria in which, unless $p(0)=\hat{p}$, the process converges to a limit cycle. Indeed, the Sonnenschein-Mantel-Debreu result on the arbitrary nature of aggregate excess demand implies that the behavior of tatonnement is also arbitrary. See Hahn (1982) for a survey of results related to tatonnement. From our point of view there are two points worth noting: First, the process can be generalized to allow different adjustment speeds

$$
p_{i}=\theta_{i} f_{i}(p), \quad i=1, \ldots, n .
$$

For any $\theta_{\mathrm{i}}>0, \mathrm{i}=1, \ldots, \mathrm{n}$, the process remains globally stable if $\mathrm{f}$ satisfies the weak axiom or gross substitutability. In general, however, changing the weights $\theta_{\mathrm{i}}$ can greatly affect the stability properties of tatonnement. Second, if we want to avoid problems with negative prices we have to alter the process to something like

$$
p_{i}= \begin{cases}f_{i}(p) & \text { if } p_{i}>0 \text { or } f_{i}(p)>0 \\ 0 & \text { otherwise }\end{cases}
$$


Although $\mathrm{p}$ can be discontinuous at a point where $\mathrm{p}_{i}=0$, it can be shown, as done for example by Henry $(1972,1973)$, that the path $p(t)$ is continuous.

Van der Laan and Talman (1987) have developed a tatonnement-like algorithm that is guaranteed to converge under weak regularity assumptions: start with an initial price vector $\overline{\mathrm{p}}$ interior to the simplex. The algorithm sets

$$
\begin{aligned}
& \mathrm{p}_{\mathrm{j}} / \overline{\mathrm{p}}_{\mathrm{j}}=\min \left[\mathrm{p}_{1} / \overline{\mathrm{p}}_{1}, \ldots, \mathrm{p}_{\mathbf{n}} / \overline{\mathrm{p}}_{\mathrm{n}}\right] \quad \text { if } \mathrm{f}_{\mathrm{j}}(\mathrm{p})<0 \\
& \mathrm{p}_{\mathrm{j}} / \overline{\mathrm{p}}_{\mathrm{j}}=\max \left[\mathrm{p}_{1} / \overline{\mathrm{p}}_{1}, \ldots, \mathrm{p}_{\mathbf{n}} / \overline{\mathrm{p}}_{\mathbf{n}}\right] \quad \text { if } \mathrm{f}_{\mathrm{j}}(\mathrm{p})>0 .
\end{aligned}
$$

When $f_{j}(p)=0, p_{j}$ is allowed to vary to keep market $j$ in equilibrium. The set of points that satisfy these conditions generically form a collection of loops and paths in $S$. The algorithm operates like the global Newton method and the path following methods described earlier, following the path that starts at $\overline{\mathrm{p}}$ until another endpoint is reached. This endpoint is an equilibrium.

Walras (1874, Lessons 12 and 24) originally conceived of tâtonnement as clearing one market at a time. With linear equations the analogous process is called the Gauss-Seidel method. The idea is to update a guess at a solution to the equations

$$
\mathrm{f}_{\mathrm{i}}\left(1, \mathrm{p}_{2}, \mathrm{p}_{3}, \ldots, \mathrm{p}_{\mathrm{n}}\right)=0, \quad \mathrm{i}=2, \ldots, \mathrm{n}
$$

one equation at a time: Given the guess $p_{2}^{k}, \ldots, p_{n}^{k}$, we let $p_{i}^{k+1}$ be the solution $g_{i}\left(p^{k}\right)$ to

$$
f_{1}\left(1, g_{2}\left(p^{k}\right), \ldots, g_{i}\left(p^{k}\right), p_{i+1}^{k}, \ldots, p_{u}^{k}\right)=0, \quad i=2, \ldots, n .
$$

In the case where $f$ is linear, this method converges if there exists some $\theta_{j}>0, j=2, \ldots, n$, such that

$$
\theta_{\mathrm{i}}\left|\frac{\partial \mathrm{f}_{\mathrm{i}}}{\partial \mathrm{p}_{\mathrm{i}}}\right| \geq \sum_{\mathrm{j} \neq \mathrm{i}} \theta_{\mathrm{j}}\left|\frac{\partial \mathrm{f}_{\mathrm{i}}}{\partial \mathrm{p}_{\mathrm{j}}}\right|, \quad \mathrm{i}=2, \ldots, \mathrm{n},
$$

with strict inequality some $i_{\text {. }}$ (See Young 1971 for a collection of conditions that guarantee convergence of this method.) This, however, is the familiar diagonal dominance condition satisfied by the Jacobian matrix of an excess demand function that exhibits gross substitutability. 
Consequently, it is possible to show that, if $\mathrm{Df}(\beta)$ satisfies gross substitutability at some equilibrium $p$, there is some open neighborhood $N$ of $p$ such that if $p^{0} \in N$, the nonlinear analog of this algorithm converges to $\mathrm{p}$. The weak axiom does not guarantee diagonal dominance, however, and it is easy to construct examples that satisfy the weak axiom but for which this method is unstable.

Perhaps the most popular method for solving systems of equations such as $g(p)=p$ is Newton's method,

$$
\mathrm{p}^{\mathrm{k}}=\mathrm{p}^{\mathrm{k}-1}-\lambda_{\mathrm{k}}\left(\mathrm{I}-\mathrm{Dg}\left(\mathrm{p}^{\mathrm{k}-1}\right)\right)^{-1}\left(\mathrm{p}^{\mathrm{k}-1}-\mathrm{g}\left(\mathrm{p}^{\mathrm{k}-1}\right)\right) .
$$

Frequently, the scalar $\lambda_{k}>0$ is chosen by a line search to make $\left\|p^{k}-g\left(p^{k}\right)\right\|$ as small as possible. Furthermore, the elements of $\mathrm{Dg}$ are usually approximated numerically rather than calculated analytically. In many versions of this algorithm $I-D g$ is never explicitly inverted. Rather, an approximation to its inverse is successively updated; these are called quasi-Newton methods. See Ortega and Rheinboldt (1970) and Jacobs (1977) for surveys of these methods. An important warning is in order here: Most work in the mathematical programming literature on Newton-type methods relates to minimizing a convex function $h: R^{n} \rightarrow R$,

$$
x^{k}=x^{k-1}-\lambda_{k} D^{2} h\left(x^{k-1}\right)^{-1} \operatorname{Dh}\left(x^{k-1}\right)^{\prime}
$$

Although this does amount to solving the system of equations $\operatorname{Dh}(x)=0$, this system has two special properties: First, $\mathcal{D}^{2} h$ is symmetric and positive semi-definite. Second, $\lambda_{k}$ can always be chosen small enough so that $h\left(x^{k}\right)$ decreases at every iteration. Unless I - Dg satisfies strong integrability conditions, these sorts of properties do not carry over to solving for equilibria.

Arrow and Hahn (1971, Chapter 12) have shown that a continuous version of Newton's method

$$
\grave{p}=-(\mathrm{I}-\mathrm{D}(\mathrm{gp}))^{-1}(p-g(\mathrm{p}))
$$


is globally stable if det $(\mathrm{A}-\mathrm{Dg}(\mathrm{p})$ ) never vanishes. (We ignore the minor technical problem caused by the potential discontinuity of $\mathrm{Dg}$; as in the case of tatonnement where some price is zero, $\mathrm{p}(\mathrm{t}) \mathrm{can}$ be shown to follow a continuous path.) In this case, the index theorem implies that there is a unique fixed point $p=g(p)$. $L(p)=(1 / 2)(p-g(p))^{\prime}(p-g(p))$ provides a Liapunov function: $L(p)>0$ unless $p=g(p)$, and

$$
\dot{L}(p)=(p-g(p))^{\prime}(I-D g(p)) \dot{p}=-(p-g(p))^{\prime}(p-g(p)) .
$$

Consequently, $\mathrm{L}<0$ unless $\mathrm{p}=\mathrm{p}$.

Although this method may cycle if $(I-D g(p))$ is singular for some $p, L(p)$ always serves as a local Liapunov function near a regular equilibrium $\hat{p}$. That is, every regular equilibrium $\hat{p}$ has some open neighborhood $\mathrm{N}$ such that, if $\mathrm{p}(0) \in \mathrm{N}$, this method converges to $\hat{p}$. This suggests a stochastic method for computing equilibria, which is frequently used in practice: Guess a value for $p(0)$. Apply Newton's method. If it does not converge, guess a new value for $p(0)$. Continue until an equilibrium is located. Since every open neighborhood of an equilibrium occupies a positive fraction of the volume of the price simplex, this method works with probability one eventually.

Newton's method is in some sense the simplest algorithm that has this local convergence property for any regular equilibrium. Saari and Simon (1978) and Traub and Wozniakowski (1976) show that, in a precise sense, any locally convergent method must use all of the information in $g(p)$ and $\mathrm{Dg}(\mathrm{p})$. Furthermore, Saari (1985) shows that for any lower bound $\bar{\lambda}$ on the step size, $\lambda_{k} \geq \bar{\lambda}>$ 0 , there are examples such that a discrete version of Newton's method is not even locally convergent. Since we cannot always choose $\lambda_{k}$ so that $\|p-g(p)\|$ is decreasing, we have to bound $\lambda_{k}$ from below so the method does not get stuck away from an equilibrium. Saari shows that this may result in the method overshooting the equilibrium.

Notice that the global Newton method has a global convergence property. It uses global information, however, because it is only guaranteed to work if started on the boundary or at an 
equilibrium with index -1 . Otherwise, it may cycle (see Keenan 1981). Notice too that the global Newton method diverges from any equilibrium with $\operatorname{det}[I-\operatorname{Dg}(\beta)]<0$. Because the scale factor $\mu(\mathrm{p})<0$ in some open neighborhood of $\mathrm{p}, \mathrm{L}(\mathrm{p})$ is actually increasing in that neighborhood.

\section{Equilibrium and Optimization}

The problem of computing an equilibrium of an economy can sometimes be simplified by transforming it into a problem of maximizing a concave function on a convex constraint set. Such transformations typically exploit the Pareto efficiency of the equilibrium allocation: Pareto (1909) first realized that the allocation $\left(\hat{\mathrm{R}}^{1}, \ldots, \mathrm{x}^{\mathrm{m}}\right)$ associated with an equilibrium $\mathrm{\beta}$ has the property that there is no alternative allocation $\left(\overline{\mathrm{x}}^{1}, \ldots, \overline{\mathrm{x}}^{\mathrm{m}}\right)$ that is superior in the sense that it is feasible, $\sum_{i=1}^{m} \overline{\mathrm{x}}^{\mathrm{i}} \leq$ $\sum \underset{i=1}{m} w^{i}$, and $u_{i}\left(\bar{x}^{i}\right) \geq u_{i}\left(\ell^{i}\right), i=1, \ldots, m$, with strict inequality for some $i$.

\subsection{Welfare Theory and Existence of Equilibrium}

That every competitive allocation is Pareto efficient is the first theorem of welfare economics. The second welfare theorem says that for any Pareto efficient allocation $\left(\hat{\mathrm{x}}^{\mathrm{l}}, \ldots, \hat{\mathrm{x}}^{\mathrm{m}}\right)$ there exists a vector of prices $p$ such that $\left(\beta, x^{1}, \ldots, x^{m}\right)$ is an equilibrium with transfer payments $t_{i}=$ $\hat{p}^{\prime}\left(\mathbf{R}^{i}-w^{i}\right)$. In other words, each consumer solves the problem of maximizing utility subject to the budget constraint $\hat{p}^{\prime} \leq \hat{p}^{\prime} w^{i}+t_{i}$ rather than his usual problem. (See Arrow 1951, Debreu 1954, and Koopmans 1957.)

For every Pareto efficient allocation $\left(\mathrm{s}^{1}, \ldots, \hat{\mathrm{x}}^{\mathrm{m}}\right)$ there exists a vector of nonnegative welfare weights $\left(\alpha_{1}, \ldots, \alpha_{\mathrm{m}}\right)$ such that $\left(\hat{\mathrm{x}}^{1}, \ldots, \hat{\mathrm{x}}^{\mathrm{m}}\right)$ solves the Pareto problem

$$
\max \sum_{i=1}^{m} \alpha_{i} u_{i}\left(x^{i}\right)
$$

subject to 


$$
\begin{aligned}
& \sum_{i=1}^{m} x^{i} \leq \sum_{i=1}^{m} w^{i} \\
& x^{i} \geq 0 .
\end{aligned}
$$

If $\alpha>0$, then any solution to this problem is Pareto efficient. The Kuhn-Tucker theorem says that the allocation $\left(\mathrm{x}^{1}(\alpha), \ldots, \mathrm{x}^{\mathrm{m}}(\alpha)\right)$ solves this problem if and only if there exists a nonnegative vector $p(\alpha)$ such that

$$
\begin{gathered}
\sum_{i=1}^{m} \alpha_{i} u_{i}\left(x^{i}(\alpha)\right)+p^{\prime} \sum_{i=1}^{m}\left(w^{i}-x^{i}(\alpha)\right) \geq \sum_{i=1}^{m} \alpha_{i} u_{i}\left(x^{i}(\alpha)\right)+p(\alpha)^{\prime} \sum_{i=1}^{m}\left(w^{i}-x^{i}(\alpha)\right) \\
\geq \sum_{i=1}^{m} \alpha_{i} u_{i}\left(x^{i}\right)+p(\alpha)^{\prime} \sum_{i=1}^{m}\left(w^{i}-x^{i}\right)
\end{gathered}
$$

for all $\mathrm{p} \geq 0$, and $\left(\mathrm{x}^{1}, \ldots, \mathrm{x}^{\mathrm{m}}\right) \geq 0$.

Similarly, each consumer solves his utility maximization problem in equilibrium if and only if there exists $\hat{\lambda} \geq 0$ such that

$$
u_{i}\left(x^{i}\right)+\lambda_{i} \hat{\rho}^{\prime}\left(w^{i}-x^{i}\right) \geq u_{i}\left(x^{i}\right)+\hat{\lambda}_{i} \hat{\rho}^{\prime}\left(w^{i}-x^{i}\right) \geq u_{i}\left(x^{i}\right)+\hat{\lambda}_{i} \hat{p}^{\prime}\left(w^{i}-x^{i}\right)
$$

for all $\lambda_{i} \geq 0$, and $x^{i} \geq 0$. Notice that the strict monotonicity of $u_{i}$ implies that $\hat{\lambda}_{i}>0$, otherwise we would violate the second inequality simply by increasing $x^{i}$. Dividing the second inequality through by $\hat{\lambda}_{i}$ and summing over $i=1, \ldots$, m produces

$$
\sum_{i=1}^{m}\left(1 \hat{\lambda_{i}}\right) u_{i}\left(\hat{x}^{i}\right)+\bar{p}^{\prime} \sum_{i=1}^{m}\left(w^{i}-\ell^{i}\right) \geq \sum_{i=1}^{m}\left(1 / \hat{\Lambda}_{i}\right) u_{i}\left(x^{i}\right)+\hat{p}^{\prime} \sum_{i=1}^{m}\left(w^{i}-x^{i}\right)
$$

for all $\left(x^{1}, \ldots, x^{m}\right) \geq 0$. Moreover, since $\beta^{\prime} \sum_{i=1}^{m}\left(w^{i}-\hat{x}^{i}\right)=0$ because of strict monotonicity and $\sum_{i=1}^{m}\left(w^{i}-x^{i}\right) \geq 0$ because of feasibility,

$$
\sum_{i=1}^{m}\left(1 \hat{\lambda_{j}}\right) u_{i}\left(\hat{x}^{i}\right)+p^{\prime} \sum_{i=1}^{m}\left(w^{i}-\hat{x}^{i}\right) \geq \sum_{i=1}^{m}\left(1 / \hat{\lambda}_{i}\right) u_{i}\left(\hat{x}^{i}\right)+\hat{p}^{\prime} \sum_{i=1}^{m}\left(w^{i}-\hat{x}^{i}\right)
$$


for all $\mathrm{p} \geq 0$. Consequently, every competitive equilibrium solves the above Pareto problem where $\alpha_{i}=1 / \hat{\lambda}_{i}, i=1, \ldots, m$, and $p(\alpha)=p$. This can be viewed as a proof of the first welfare theorem. Notice, too, that, if $\left(\mathrm{x}^{1}(\alpha), \ldots, \mathrm{x}^{\mathrm{m}}(\alpha)\right)$ is a solution to the Pareto problem for arbitrary nonnegative welfare weights $\alpha$, it must be the case that

$$
\alpha_{i} u_{i}\left(x^{i}(\alpha)\right)+p(\alpha)^{\prime}\left(x^{i}(\alpha)-x^{i}(\alpha)\right) \geq \alpha_{i} u_{i}\left(x^{i}\right)+p(\alpha)^{\prime}\left(x^{i}(\alpha)-x^{i}\right)
$$

for all $x^{i} \geq 0$. Otherwise the allocation that replaces $x^{i}(\alpha)$ with the $x^{i}$ that violates this inequality but leaves $\mathrm{x}^{\mathrm{j}}(\alpha), \mathrm{j} \neq \mathrm{i}$, unchanged would violate the conditions required for $\left(\mathrm{x}^{\mathrm{l}}(\alpha), \ldots, \mathrm{x}^{\mathrm{m}}(\alpha)\right)$ to solve the Pareto problem. Since $\mathrm{p}^{\prime}\left(\mathrm{x}^{\mathrm{i}}(\alpha)-\mathrm{x}^{\mathrm{i}}(\alpha)\right)=0$ for all $\mathrm{p}$, this implies that any solution to the Pareto problem is such that, if $\alpha_{\mathrm{i}}>0, \mathrm{x}^{\mathrm{i}}(\alpha)$ maximizes $\mathrm{u}_{\mathrm{i}}(\mathrm{x})$ subject to $\mathrm{p}(\alpha)^{\prime} \mathrm{x} \leq \mathrm{p}(\alpha)^{\prime} \mathrm{x}^{\mathrm{i}}(\alpha)$. Ignoring for a moment the possibility that $\alpha_{i}=0$, some $i$, we can compute the transfer payments needed to decentralize the allocation $\left(\mathrm{x}^{1}(\alpha), \ldots, \mathrm{x}^{\mathrm{m}}(\alpha)\right)$ as an equilibrium with transfer payments $t_{i}(\alpha)=p(\alpha)^{\prime}\left(x^{i}(\alpha)-w^{i}\right), i=1, \ldots, m$.

Suppose now that $\alpha_{i}=0$, some $i$. Then our earlier argument implies $0 \geq p(\alpha)^{\prime}\left(x^{i}(\alpha)-x^{i}\right)$ for all $x^{i} \geq 0$. Combined with $p(\alpha) \geq 0$, this implies that $p(\alpha)^{t} x^{i}(\alpha)=0$, that consumers with zero weight in the welfare function receive nothing of value at a solution to the Pareto problem. Since the strict monotonicity of $u_{i}$ implies $p(\alpha) \neq 0$ and since $w^{i}>0$, we know that $t_{i}(\alpha)<0$ if $\alpha_{i}=0$.

Our arguments have produced the following characterization of equilibria:

Proposition 3.1. A price-allocation pair $\left(\hat{\beta}, \mathrm{x}^{\mathrm{l}}, \ldots, \hat{\mathrm{x}}^{\mathrm{m}}\right)$ is an equilibrium if and only if there exists a strictly positive vector of welfare weights $\left(\hat{\alpha}_{1}, \ldots, \hat{\alpha}_{m}\right)$ such that $\left(\hat{\mathrm{x}}^{1}, \ldots, \mathrm{x}^{\mathrm{m}}\right)$ solves the Pareto problem with these welfare weights, that $\mathbf{p}$ is the corresponding vector of Lagrange multipliers, and that $p^{\prime}\left(\hat{x}^{i}-w^{i}\right)=0, i=1, \ldots, m$.

Bemark. The assumption that $w^{i}$ is strictly positive serves to ensure that the consumer has strictly positive income in any equilibrium and, hence, has a strictly positive welfare weight. Weaker 
conditions such as McKenzie's $(1959,1961)$ irreducibility condition ensure the same thing. Unless there is some way to ensure that the consumer has positive income, or, with more general consumption sets, can afford a consumption bundle interior to his consumption set, we may have to settle for existence of a quasi-equilibrium rather than an equilibrium. In a quasi-equilibrium each consumer minimizes expenditure subject to a utility constraint rather than maximizing utility subject to a budget constraint.

Unless we are willing to assume that $u_{i}, i=1, \ldots, m$, is continuously differentiable, there may be more than one price vector $p(\alpha)$ that supports a solution to the Pareto problem because of kinks in $u_{i}$. This makes $t(\alpha)$ a point-to-set correspondence. Nevertheless, it is still easy to prove the existence of equilibrium using an approach due originally to Negishi (1960).

Proposition 3.2 (Negishi 1960). There exists a strictly positive vector of utility weights $\left(\hat{\alpha}_{1}, \ldots, \hat{\alpha}_{\mathrm{m}}\right)$ such that $0 \in \mathrm{t}(\hat{\alpha})$.

Proof. The strict concavity of each $u_{i}, i=1, \ldots, m$, and continuity of $\sum_{i=1}^{m} \alpha_{i} u_{j}\left(x^{i}\right)$ in $\alpha$ implies that $x^{i}: R_{+}^{m} \backslash\{0\} \rightarrow R^{n}$ is a continuous function. Furthermore, $p: R_{+}^{m} \backslash\{0\} \rightarrow R^{n}$ is a nonempty, bounded, upper-hemi-continuous, convex-valued correspondence. Consequently, the correspondence $t: R_{+}^{m}\{0\} \rightarrow R^{m}$ defined by the rule

$$
\mathrm{t}_{\mathrm{i}}(\alpha)=\mathrm{p}(\alpha)^{\prime}\left(\mathrm{x}^{\mathrm{i}}(\alpha)-\mathrm{w}^{\mathrm{i}}\right)
$$

is also nonempty, bounded, upper-hemi-continuous, and convex-valued. It is homogeneous of degree one since $\mathrm{x}^{\mathrm{i}}(\alpha)$ is homogeneous of degree zero and $\mathrm{p}(\alpha)$ is homogeneous of degree one. It also obeys the identity

$$
\sum_{i=1}^{m} t_{j}(\alpha) \equiv p(\alpha)^{\prime} \sum_{i=1}^{m}\left(x^{i}(\alpha)-w^{\hat{i}}\right) \equiv 0
$$


Let $S \subset R^{m}$ now be the simplex of utility weights. Since $S$ is compact, $t$ is bounded and upper-hemi-continuous, and $\mathrm{t}_{\mathrm{i}}(\alpha)<0$ if $\alpha \in \mathrm{S}$ with $\alpha_{\mathrm{i}}=0$, there exists $\theta>0$ such that

$$
g(\alpha)=\alpha-\theta t(\alpha)
$$

defines a nonempty, upper-hemi-continuous, convex-valued correspondence g: $S \rightarrow S$. By Kakutani's fixed point theorem there exists $\hat{\alpha} \in \mathrm{g}(\hat{\alpha})$. This implies that $0 \in \mathrm{t}(\hat{\alpha})$.

Remark. The correspondence $f: R_{+}^{m} \backslash\{0\} \rightarrow R^{m}$ defined by the rule $f_{i}(\alpha)=-t_{i}(\alpha) / \alpha_{i}$ has all of the properties of the excess demand correspondence of an exchange economy with $\mathrm{m}$ goods.

\subsection{Computation and Multiplicity of Equilibria}

Negishi's approach provides an alternative system of equations $\alpha=\mathrm{g}(\alpha)$, that can be solved to find equilibria. Mantel (1971), for example, proposes a tâtonnement procedure $\alpha=-t(\alpha)$ for computing equilibria. Similarly, we could apply Scarf's algorithm, the global Newton method, a path following method, the nonlinear Gauss-Seidel method, or Newton's method to compute the equilibrium values of $\alpha$.

We have reduced the problem of computing equilibria of an economy specified in terms of preferences and endowments to yet another fixed point problem. The obvious question, in analogy to Uzawa's (1962) result, is whether any arbitrary $\mathrm{g}: \mathrm{S} \rightarrow \mathrm{S}, \mathrm{S} \subset \mathrm{R}^{\mathrm{m}}$ can be converted into a transfer function $t(\alpha)$. The answer is obviously yes if the only properties that $t$ needs to satisfy are continuity, homogeneity of degree one, and summation to zero. Bewley (1980), in fact, proves the analog of the Sonnenschein-Mantel-Debreu theorem in the case where $t$ is twice continuously differentiable and $\mathrm{n} \geq 2 \mathrm{~m}$ : for any such transfer function $\mathrm{t}$ there is an economy with $\mathrm{m}$ consumers and $\mathrm{n}$ goods that generates it. In closer analogy with the Sonnenschein-Mantel-Debreu theorem, however, it is natural to conjecture that this result holds for $\mathrm{t}$ continuous and $\mathrm{n} \geq \mathrm{m}$. 
Example 3.1 (2.1 Revisited). The Pareto problem for the exchange economy with two goods in Example 2.1 is

$$
\max \alpha_{1} \sum_{j=1}^{2} a_{j}^{1}\left(\left(x_{j}^{1}\right)^{b}-1\right) / b+\alpha_{2} \sum_{j=1}^{2} a_{j}^{2}\left(\left(x_{j}^{2}\right)^{b}-1\right) / b
$$

subject to

$$
\begin{aligned}
& x_{j}^{1}+x_{j}^{2} \leq w_{j}^{1}+w_{j}^{2}, j=1,2 \\
& x_{j}^{i} \geq 0 .
\end{aligned}
$$

(It is only in the case $b_{1}=b_{2}$ that we can obtain an analytical expression for the transfer functions.) The first order conditions for this problem are

$$
\alpha_{\mathrm{i}} \mathrm{a}_{\mathrm{j}}^{\mathrm{i}}\left(\mathrm{x}_{\mathrm{j}}^{\mathrm{j}}\right)^{\mathrm{b}-1}-\mathrm{p}_{\mathrm{j}}=0, \quad \mathrm{i}=1,2 ; \mathrm{j}=1,2 .
$$

These are, of course, the same as those of the consumers utility maximization problem when we set $\alpha_{i}=1 / \lambda_{i}$. The difference is that here the feasibility conditions are imposed as constraints, and we want to find values of $\alpha_{i}$ so that the budget constraints are satisfied. In the previous section the budget constraints were imposed as constraints, and we wanted to find values of $p_{j}$ so that the feasibility constraints were satisfied.

The solution to the Pareto problem is

$$
x_{j}^{i}(\alpha)=\frac{\left(\alpha_{i} a_{j}^{i}\right)^{\eta} \sum_{k=1}^{2} w_{j}^{k}}{\sum_{k=1}^{2}\left(\alpha_{k} a_{j}^{k}\right)^{\eta}}, i=1,2, j=1,2 .
$$

Here, once again, $\eta=1 /(1-b)$. The associated Lagrange multipliers are

$$
p_{j}(\alpha)=\frac{\left(\sum_{k=1}^{2}\left(\alpha_{k} a_{j}^{k}\right)^{\eta}\right)^{1 / \eta}}{\left(\sum_{k=1}^{2} w_{j}^{k}\right)^{1 / \eta}} .
$$


The only way that we can find this problem, however, is to compute the equilibrium by some other means. Another point worth noting is that the Pareto problems that we have considered are convex problems, which have unique solutions that are easy to verify as solutions and relatively easy to compute. Any fixed point problem, and hence any equilibrium problem, can be recast as an optimization problem,

$$
\min \|p-g(p)\|^{2}
$$

Because the objective function is not convex, however, it is relatively difficult to compute equilibria using this formulation. Nevertheless, this problem does have one aspect that makes the solution easier than that of other nonconvex optimization problems: Although we may possibly get stuck at a local minimum, at least we know what the value of the objective function is at the global minimum, $\|p-g(\hat{p})\|^{2}=0$

A recent development that may allow relatively efficient solution to nonconvex optimization problems is the simulated annealing algorithm. This algorithm, developed by Kirkpatrick, Gelatt, and Vecchi (1983), is based on the analogy between the simulation of the annealing of solids and the solution of combinational optimization problems. (See van Laarhoven and Aarts 1988 for a survey and references.) Although this method has been applied principally to combinatorial problems, which involve discrete variables, there have been some applications to continuous optimization problems (see, for example, Vanderbilt and Louie 1984 and Szu and Hartley 1987). So far, this method has not been applied to solve economic problems, however, and it remains an intriguing direction for future research.

\section{Static Production Economies}

We can add a production technology to our model in a variety of ways. Perhaps the easiest, and in many ways the most general, is to specify the production technology as a closed, convex cone 
$Y \subset R^{n}$. If $y \in Y$, then $y$ is a feasible production plan with negative components corresponding to to inputs and positive components to outputs. We assume that $-R_{+}^{n} \subset Y$, which means that any good can be freely disposed, and that $Y \cap \mathrm{R}_{+}^{\mathbf{n}}=\{0\}$, which means that no outputs can be produced without inputs.

The production cone specification assumes constant returns to scale. With the introduction of fixed factors, it can also account for decreasing returns. It cannot account for increasing returns, however, which are not compatible with the competitive framework that we employ here. See Brown (1989) for a survey of results for economies with increasing returns.

\subsection{Existence of Equilibrium}

In an economy in which consumers are specified in terms of utility functions and endowment vectors, an equilibrium is now a price vector $\hat{p} \in \mathrm{R}_{+}^{\mathrm{n}} \backslash\{0\}$, an allocation $\left(\hat{x}^{1}, \ldots, \mathrm{x}^{\mathrm{m}}\right)$, where $\hat{x}^{\mathrm{i}} \in$ $\mathbf{R}_{+}^{\mathbf{n}}$, and a production plan $g \in \mathrm{Y}$ such that

- $\mathrm{x}^{\mathrm{i}}, \mathrm{i}=1, \ldots, \mathrm{m}$, solves

$\max u_{i}(x)$

subject to

$$
\begin{aligned}
& \beta^{\prime} x \leq \rho^{\prime} w^{i} \\
& x \geq 0
\end{aligned}
$$

- $\hat{p}^{\prime} \hat{y}=0$ and $\hat{p}^{\prime} y \leq 0$ for all $y \in Y$,

$\cdot \sum_{i=1}^{m} x^{i}=y+\sum_{i=1}^{m} w^{i}$

The second condition is the familiar profit maximization condition for a constant-returns technology. In an economy in which consumers are specified in terms of an aggregate excess demand function, an equilibrium is now a price vector $\beta \in R_{+}^{\mathrm{a}} \backslash\{0\}$ such that 
- $\mathrm{p}^{\prime} \mathrm{y} \leq 0$ for all $y \in Y$,

- $f(\hat{p}) \in Y$.

Notice that Walras's law implies that $y=f(p)$ is such that $\hat{p}^{\prime} y=0$.

Specifying the production technology as a cone is often too abstract an approach for many applications. Alternatively, it may be specified by an $n \times k$ activity analysis matrix $A$, where each column of A represents a feasible production plan. (See, for example, Koopmans 1951.) In this case

$$
Y=\left\{x \in R^{n} \mid x=A y, \text { some } y \in R_{+}^{k}\right\},
$$

and $\mathrm{Y}$ is the polyhedral cone spanned by $\mathrm{k}$ columns of $\mathrm{A}$. Another alternative is to specify production in terms of a finite number of concave, homogeneous-of-degree-one production functions $h_{j}: R^{n} \rightarrow R, j=1, \ldots, k$. In this case

$$
Y=\left\{x \in R^{n} \mid x=\sum_{j=1}^{k} z^{j}, h_{j}\left(z^{j}\right) \geq 0, j=1, \ldots, k\right\} .
$$

For example, $h\left(z_{1}, z_{2}, z_{3}\right)=\eta\left(-z_{1}\right)^{\theta}\left(-z_{2}\right)^{1-\theta}-z_{3}$ is the familiar Cobb-Douglas production function. (Of course, the activity analysis specification is a special case of this one since, for example, $h\left(z_{1}, z_{2}, z_{3}\right)=\min \left[z_{1} / a_{1 j}, z_{2} / a_{2 j}\right]-z_{3} / a_{3 j}$ is a concave production function.)

Another aiternative is to allow decreasing returns to scale, where the production function $h_{j}$ is strictly concave. In this case, the problem of maximizing $\mathrm{p}^{\prime} z$ subject to $h_{j}(z) \geq 0$ has a unique solution $z^{j}(p)$. Unless $z^{j}(p)=0$ there are positive profits $\pi_{j}(p)=p^{\prime} z^{j}(p)$ that must be spent. Letting $\theta_{1}^{\mathrm{j}} \geq 0, \sum_{\mathrm{i}=1}^{\mathrm{m}} \theta_{1}^{\mathrm{j}}=1, \mathrm{j}=1, \ldots, \mathrm{k}$, be profit shares, we change the budget constraint of consumer $\mathrm{i}$ to $p^{\prime} x \leq p^{\prime} w^{i}+\sum_{j=1}^{k} \theta i \pi_{j}(p)$. We could define the excess demand function to include production responses,

$$
f(p)=\sum_{i=1}^{m}\left(x^{i}(p)-w^{i}\right)-\sum_{j=1}^{k} z^{j}(p)
$$


Although we have to restrict ourselves to the convex set of prices for which $\pi_{j}(p)<\infty, j=1$, $\ldots, k$, this approach is frequently very convenient for computation. From a theoretical viewpoint, however, it is easier to view even this as a special case of constant returns, defining a new good, an inelastically supplied factor of production, to account for each industry's profits and endowing consumers with this factor in the proportions $\theta_{j}^{i}$ (see, for example, McKenzie 1959).

A simple extension of our previous argument demonstrates the existence of equilibrium in a production technology and suggests algorithms for computing equilibria. Again we use homogeneity to normalize prices so that $e^{\prime} p=1$. Consider the set

$$
S_{Y}=\left\{p \in R^{n} \mid e^{\prime} p=1, p^{\prime} y \leq 0 \text {, all } y \in Y\right\} .
$$

$S_{Y}$ is obviously closed and convex. Since $-R_{+}^{\mathfrak{n}} \subset Y$ implies that $S_{Y} \subset S$, it is compact. Since $Y \cap R_{+}^{\mathbf{n}}=\{0\}$, the separating hyperplane theorem implies that there exists $\overline{\mathbf{p}} \not \mathbf{0}$ such that $\overline{\mathrm{p}}^{\prime} \mathrm{y} \leq 0$ for all $y \in Y$. This implies that $S_{Y}$ is nonempty. Now let $\mathrm{g}: S_{Y} \rightarrow S_{Y}$ be the function that associates any point $p \in S_{Y}$ with the point $g(p)$ that is the closest point in $S_{Y}$ to $p+f(p)$ in terms of euclidean distance. In other words, $g(p)$ solves the problem

$$
\min (1 / 2)(g-p-f(p))^{\prime}(g-p-f(p))
$$

subject to

$$
\mathbf{g} \in \mathrm{S}_{\mathbf{Y}}
$$

Once again, since the objective function is strictly convex in $\mathrm{g}$ and continuous in $\mathrm{p}$ and the constraint set is convex, $g(p)$ is a continuous function.

Proposition 4.1 (Eaves 1971 and Todd 1979). $\hat{p}$ is an equilibrium if and only if it is a fixed point of $g, p=g(\hat{p})$.

Proof. $g(p)$ solves the minimization problem that defines it if and only if

$$
(g(p)-p-f(p))^{\prime} g(p) \leq(g(p)-p-f(p))^{\prime} q
$$




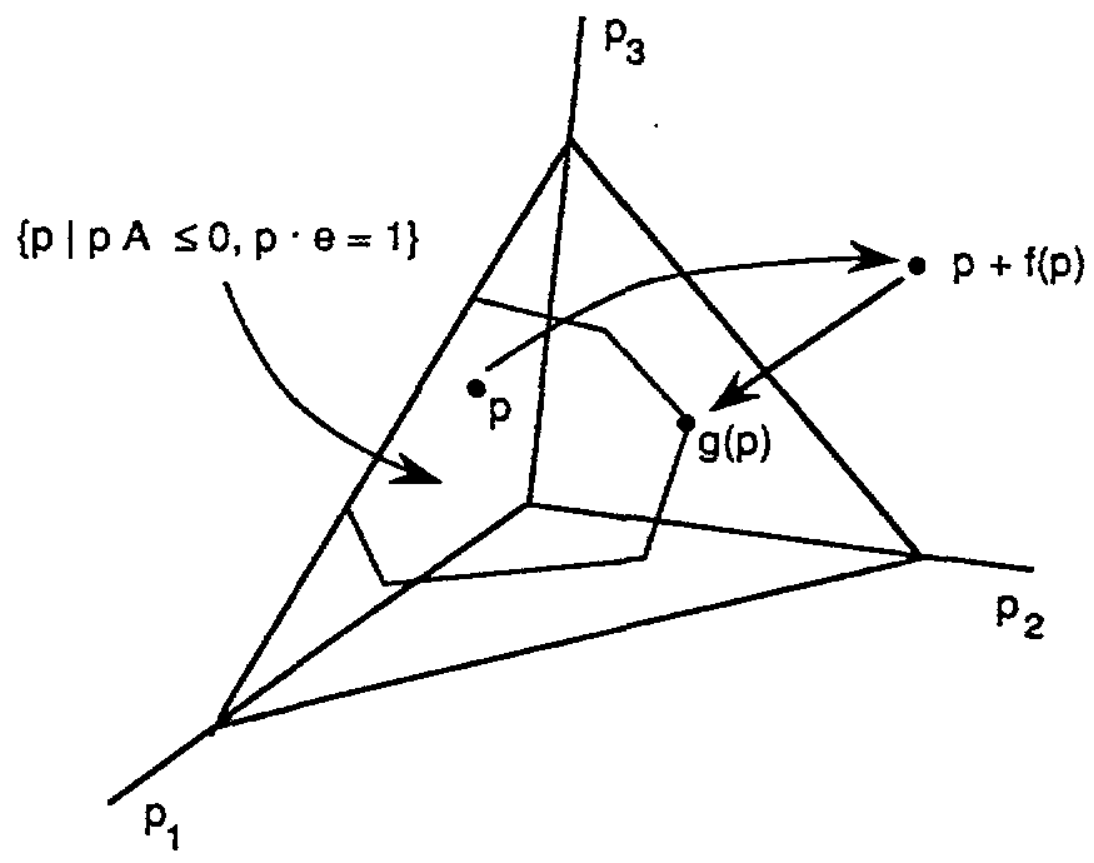

FIGURE 4.1 
for all $q \in S_{Y}$. If $(p+f(p)) \notin S_{Y}$, then this inequality says that the hyperplane passing through $g$ with normals $(g(p)-p-f(p))$ separates $g(p)$ from $S_{Y}$. If $(p+f(p)) \in S_{Y}$, then $g(p)=$ $p+f(p)$ and the inequality is trivial.

Suppose that $p=g(\hat{p})$. Then the above inequality becomes $q^{\prime} f(\hat{p}) \leq \hat{p}^{\prime} f(\hat{p})=0$ for all $p \in S_{Y}$. Since we can multiply this inequality by any positive constant without changing it, we obtain $q^{\prime} f(p) \leq 0$ for all $q \in Y^{*}$, where $Y^{*}=\left\{q \in R^{n} \mid q^{\prime} y \leq 0\right.$, all $\left.y \in Y\right\}$ is the dual cone of $Y$. This says that $f(\hat{\beta})$ is an element of the dual cone of $Y^{*}, Y^{* *}=\left\{y \in R^{n} \mid y^{\prime} q \leq 0\right.$, all $\left.\mathrm{q} \in \mathrm{Y}^{*}\right\}$. Since $\mathrm{Y}$ is convex, $\mathrm{Y}^{* *}=\mathrm{Y}$. Consequently, $\hat{\mathrm{p}}$ is an equilibrium.

Conversely, if $\hat{p}$ is an equilibrium, then $q^{\prime} f(\beta)$ for all $q \in S_{Y}$, which implies that $g(\hat{p})=\hat{p}$.

Remark. Once again, this approach can be used to deal with demand correspondences rather than functions. Again letting $D$ be the convex hull of the image of $p+f(p)$ for $p \in S_{Y}$, we define $\mathrm{q}: \mathrm{D} \rightarrow \mathrm{S}_{\mathrm{Y}}$ as the least-squares projection onto $\mathrm{S}_{\mathrm{Y}}$. As in the exchange case, the correspondence $g: D \rightarrow D$ defined by the rule $g(p)=q(p)+f(q(p))$ satisfies the conditions required by Kakutani's fixed point theorem to have a fixed point $\hat{\rho} \in \mathrm{g}(\hat{\phi})$, and $\hat{\mathrm{p}}$ is a fixed point if and only if it is equilibrium.

Computing equilibria by computing fixed points of the function $g$ is easiest when the production technology is specified by an activity analysis matrix. In that case, $g(p)$ can be calculated by solving the quadratic progranming problem

$$
\min (1 / 2)(g-p-f(p))^{\prime}(g-p-f(p))
$$

subject to

$$
\begin{aligned}
& A^{\prime} g \leq 0 \\
& e^{\prime} g=1
\end{aligned}
$$


Let $B$ be the $n \times \ell$ matrix of columns of A associated with strictly positive Lagrange multipliers $y_{j}$ in the first-order conditions

$$
g-p-f(p)+A y+\lambda e=0 .
$$

Let $\mathrm{C}$ be the $\mathrm{n} \times(\ell+1)$ matrix [B e], and let $\mathrm{e}^{\ell+1}$ be the $(\ell+1) \times 1$ vector with $e_{\ell+1}^{\ell+1}=1$ and $e_{j}^{\ell+1}=0, j \neq \ell+1$. Then elementary matrix manipulation yields an explicit formula for $g(p)$ :

$$
g(p)=\left(1-C\left(C^{\prime} C\right)^{-1} C^{\prime}\right)(p+f(p))+C\left(C^{\prime} C\right)^{-1} e^{\ell+1} .
$$

Furthermore, the matrix $C$ changes only when a Lagrange multiplier $y_{j}$ that has been previously positive becomes zero or one that has been previously zero becomes positive.

Suppose that the production technology specified by $\mathrm{k}$ concave production functions. Let $a_{j}(p)$ be the restricted profit corresponding to the production function $h_{j}(z)$, the value of the objective function at the solution to

$$
\max p^{\prime} z
$$

subject to

$$
\begin{gathered}
h_{j}(z) \geq 0 \\
\|z\|=1 .
\end{gathered}
$$

(For production functions with only one output $i$ a restriction like $z^{i}=1$ is more natural than $\|z\|=1$.) If this problem has a unique solution $z^{j}(p)$, then $a_{j}(p)$ is continuously differentiable and, by Hotelling's lemma, $\mathrm{Da}_{j}(p)=z^{j}(p)^{\prime}$ (see, for example, Diewert 1982). In this case $g(p)$ is found by solving

$$
\min (1 / 2)(g-p-f(p))^{\prime}(g-p-f(p))
$$

subject to

$$
\begin{aligned}
& a_{j}(g) \leq 0, j=1, \ldots, k \\
& e^{\prime} g=1 .
\end{aligned}
$$


Because the constraints are nonlinear, however, solving this problem is more difficult than it is in the activity analysis case.

\subsection{The Index Theorem and Multiplicity of Equilibria}

Mas-Colell $(1975,1985)$ and Kehoe $(1980,1983)$ have extended the concepts of regularity and fixed point index to production economies. They prove that regular production economies have the same desirable properties as regular exchange economies and that, in a precise sense, almost all economies are regular. Kehoe (1980) further calculates the index of a regular equilibrium of an economy with an activity analysis production technology as

$$
\operatorname{index}(\hat{\rho})=\operatorname{sgn}\left[\operatorname{det}\left[\begin{array}{cc}
-\bar{J} & \bar{B} \\
-\bar{B}^{\prime} & 0
\end{array}\right]\right) \text {. }
$$

Here $\overline{\mathrm{B}}$ is the $(\mathrm{n}-1) \times \ell$ matrix formed by deleting the first row from the matrix of activities used at equilibrium $\hat{p}$. In the case of more general profit functions, let $H(\hat{\rho})$ by the $n \times n$ weighted sum of the Hessian matrices of profit functions used at $\rho, H(\rho)=\sum_{j=1}^{k} \hat{q}_{j} D^{2} a_{j}(\hat{p})$; the weights $\oint_{j}$ are the appropriate activity levels. In this case, Kehoe (1983) calculates

$$
\text { index }(\hat{\boldsymbol{\beta}})=\operatorname{sgn}\left(\operatorname{det}\left[\begin{array}{ll}
-\overrightarrow{\mathrm{J}}+\overline{\mathrm{H}} & \overline{\mathrm{B}} \\
-\overline{\mathrm{B}}^{\prime} & 0
\end{array}\right]\right) \text {. }
$$

where $\overline{\mathrm{H}}$ is the $(n-1) \times(n-1)$ matrix formed by deleting the first row and column from $H(\hat{p})$.

Once again, if $\mathrm{f}$ satisfies the weak axiom, the set of equilibria is convex, and in the regular case there is a unique equilibrium. Unfortunately, even if $f$ exhibits gross substitutability, the economy need not have a unique equilibrium, as the following example illustrates.

Example 4.1. Consider a static production economy with two consumers and four goods. Consumer $i, i=1,2$ has a utility function of the form 


$$
u_{i}\left(x_{1}, x_{2}, x_{3}, x_{4}\right)=\sum_{j=1}^{4} a_{j}^{i} \log x_{j}
$$

where $a_{j}^{\dot{i}} \geq 0$. Given an endowment vector $\left(w_{1}^{i}, w_{2}^{i}, w_{3}^{i}, w_{4}^{j}\right)$, consumer $i$ maximizes this utility function subject to his budget constraint. The aggregate excess demand function is

$$
f_{j}\left(p_{1}, p_{2}, p_{3}, p_{4}\right)=\sum_{i=1}^{2}\left[\frac{a_{j}^{i} \sum_{k=1}^{4} p_{k} w_{k}^{i}}{p_{j} \sum_{k=1}^{4} a_{k}^{i}}\right], \quad j=1,2,3,4 .
$$

The consumers have the following parameters:

\begin{tabular}{|c|c|c|c|c|}
\hline \multirow[b]{2}{*}{ Consumer } & \multicolumn{2}{|c|}{$\begin{array}{c}w_{j}^{\mathrm{i}} \\
\text { Commodity }\end{array}$} & \multirow[b]{2}{*}{3} & \multirow[b]{2}{*}{4} \\
\hline & 1 & 2 & & \\
\hline 1 & 0 & 0 & 10 & 0 \\
\hline 2 & 0 & 0 & 0 & 20 \\
\hline
\end{tabular}

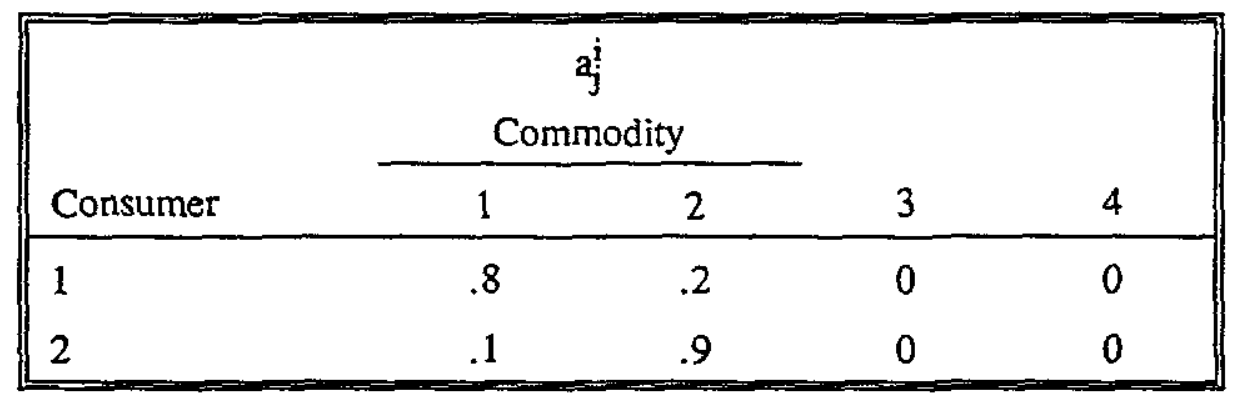

The production technology is specified by a $4 \times 8$ activity analysis matrix

$$
A=\left[\begin{array}{rrrrrrrr}
-1 & 0 & 0 & 0 & 3 & 5 & -1 & -1 \\
0 & -1 & 0 & 0 & -1 & -1 & 5 & 5 \\
0 & 0 & -1 & 0 & -1 & -1 & -1 & -4 \\
0 & 0 & 0 & -1 & -1 & -4 & -3 & -1
\end{array}\right]
$$

(The first four columns are disposal activities.) 
This economy has three equilibria, which are listed below together with the welfare weights for which the corresponding Pareto problem yields the same equilibrium:

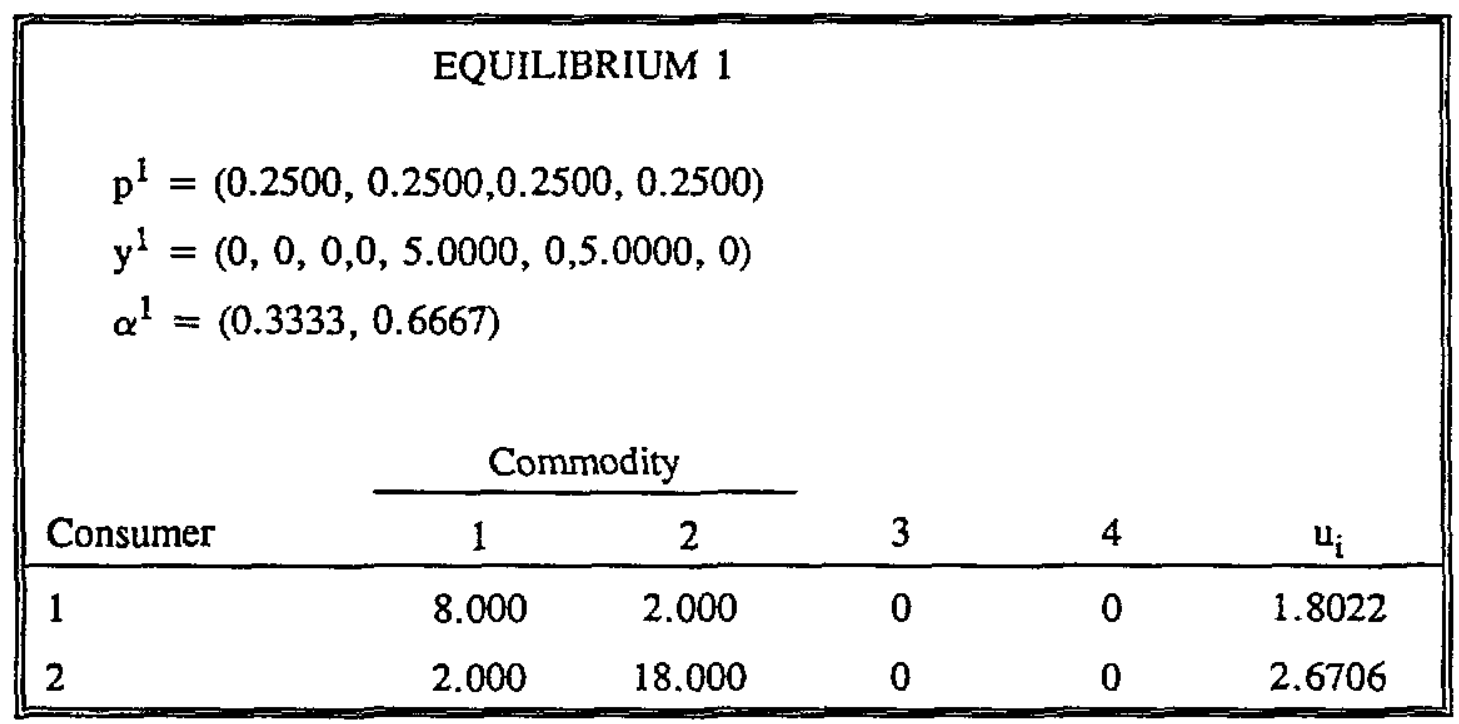

\begin{tabular}{|c|c|c|c|c|c|}
\hline \multicolumn{6}{|c|}{$\begin{array}{l}\mathrm{p}^{2}=(0.2500,0.2222,0.3611,0.1667) \\
\mathrm{y}^{2}=(0,0,0,0,5.1806,0.3611,4.4583,0) \\
\alpha^{2}=(0.5200,0.4800)\end{array}$} \\
\hline \multirow[b]{2}{*}{ Consumer } & \multicolumn{2}{|c|}{ Commodity } & & & \\
\hline & 1 & 2 & 3 & 4 & $u_{i}$ \\
\hline 1 & 11.555 & 3.250 & 0 & 0 & 2.1935 \\
\hline 2 & 1.333 & 13.500 & 0 & 0 & 2.3719 \\
\hline
\end{tabular}




\begin{tabular}{|c|c|c|c|c|c|}
\hline $\begin{array}{l}\mathrm{p}^{3}= \\
\mathrm{y}^{3}= \\
\alpha^{3}=\end{array}$ & $\begin{array}{l}708,0 \\
4.3690 \\
7895)\end{array}$ & $\begin{array}{l}\text { QUILIBR } \\
0.3125 \\
1548\end{array}$ & & & \\
\hline & & dity & & & \\
\hline Consumer & 1 & 2 & 3 & 4 & $\mathrm{u}_{\mathrm{i}}$ \\
\hline 1 & 5.333 & 1.231 & 0 & 0 & 1.3807 \\
\hline 2 & 2.500 & 20.769 & 0 & 0 & 2.8217 \\
\hline
\end{tabular}

This example has been constructed by making $\mathrm{p}^{1}=(0.25,0.25,0.25,0.25)$ be an equilibrium with index $\left(p^{1}\right)=-1$.

$$
\begin{gathered}
\operatorname{Df}\left(p^{1}\right)=\left[\begin{array}{rrrr}
-40 & 0 & 32 & 8 \\
0 & -80 & 8 & 72 \\
0 & 0 & 0 & 0 \\
0 & 0 & 0 & 0
\end{array}\right] \\
\text { index }\left(p^{1}\right)=\operatorname{sgn}\left[\operatorname{det}\left[\begin{array}{rrrrr}
80 & -8 & 72 & -1 & 5 \\
0 & 0 & 0 & -1 & -1 \\
0 & 0 & 0 & -1 & -3 \\
1 & 1 & 1 & 0 & 0 \\
-5 & 1 & 3 & 0 & 0
\end{array}\right]\right) \\
\text { index }\left(p^{1}\right)=\operatorname{sgn}(-416)=-1 .
\end{gathered}
$$

That this example has multiple equilibria means that gross substitutability does not imply the weak axiom. Kehoe and Mas-Colell (1984) prove that gross substitutability does indeed imply the weak axiom if $\mathrm{n} \leq 3$, so at least four goods are needed for this example. Kehoe (1985b) presents an example with multiple equilibria in which the $f$ exhibits strong gross substitutability, $\partial \mathrm{f}_{\mathrm{i}} / \partial \mathrm{p}_{\mathrm{j}}>0$. The simple form of a Jacobian matrix in this example, which satisfies gross substitutability but 
implies a violation of the weak axiom is due to Mas-Colell (1986) (see also Hildenbrand 1989 and Kehoe 1986).

Unfortunately, as Herbert Scarf has demonstrated to the author, the weak axiom is the weakest condition on $\mathrm{f}$ that guarantees that a production economy has a unique equilibrium: Suppose that there are two distinct price vectors $p$ and $q$ such that $p^{\prime} f(q) \leq 0$ and $q^{\prime} f(p) \leq 0$. Let

$$
Y=\left\{x \in R^{n} \mid x \leq f(p) y_{1}+f(q) y_{2}, y_{j} \geq 0\right\}
$$

Then both $p$ and $q$ are equilibria of $(f, Y)$. Aggregation conditions like those of AntonelliGorman-Nataf and Eisenberg-Chipman guarantee that $f$ satisfies the strong axiom of revealed preference, a much stronger condition than the weak axiom. To make further progress on developing economically meaningful conditions that guarantee uniqueness of equilibrium, we need to develop economically meaningful conditions that imply that the weak axiom holds in the aggregate. A step in this direction has been taken by Hildenbrand (1983), who places restrictions on income distribution in an economy with a continutum of agents that imply the weak axiom. See Jerison (1984) for more results in this same direction.

\subsection{A Tâtonnement Method}

The function $g$ whose fixed points are equilibria suggests a tatonnement process for computing equilibria in production economies: Let $\bar{B}(p)$ be the $n \times \bar{l}$ matrix columns are those vectors $\mathrm{Da}_{j}(\mathrm{p})$ such that $\mathrm{a}_{j}(\mathrm{p})=0$. Let $p$ be the vector $q$ that solves

$$
\min (1 / 2)(q-f(p))^{\prime}(q-f(p))
$$

subject to

$$
\tilde{\mathrm{B}}^{\prime}(\mathrm{p}) \mathrm{q} \leq 0
$$

Suppose that we start this process at $p(0) \in R^{n}$ such that $a_{j}(p(0)) \leq 0, j=1, \ldots, k$. Unfortunately, $p$ is discontinuous when $p(t)$ runs into a new constraint $a_{j}(p(t))=0$. This is the same problem as 
that in the exchange case where some coordinate of $p_{i}(t)$ becomes zero, however, and it can be argued that $p(t)$ is continuous in spite of it. Notice that, since $d / d t\left(a_{j}(p)\right)=D_{a_{j}}(p) p$ and $D_{j}(p) p \leq 0$ whenever $a_{j}(p(t))=0, p^{(t)}$ stays in $Y^{*}=\left\{p \in R^{n} \mid a_{j}(p) \leq 0, j=1, \ldots, k\right\}$. Notice too that, if $\dot{p}=0$ at $\vec{p}$, then $\hat{p}$ is an equilibrium: $q$ solves the above problem if and only if there exists a nonnegative $\tilde{\ell} \times 1$ vector of Lagrange multipliers y such that

$$
\begin{aligned}
& q-f(p)+\tilde{B}(p) y=0 \\
& y^{\prime} \tilde{B}^{\prime}(p) q=0 .
\end{aligned}
$$

At $q=0$ this becomes $f(\hat{p})=\tilde{B}(\hat{p}) y$.

Let $B(p)$ be the $n \times \ell$ submatrix of $\bar{B}$ whose columns are associated with strictly positive Lagrange multipliers. Then we can solve explicitly for $q$ to obtain

$$
p=\left(I-B(p)\left(B^{\prime}(p) B(p)\right)^{-1} B^{\prime}(p)\right) f(p) .
$$

Like the tâtonnement process for exchange economies, this process leaves $\|\mathrm{p}\|$ constant. Also like the tatonnement process for exchange economies, this process is globally asymptotically stable if the weak axiom of revealed preference holds in comparisons between arbitrary price vectors $p$ and the equilibrium price vector $\hat{p}$. Suppose that $p \in Y^{*}$. Then $f(\hat{p}) \in Y$ implies $p^{\prime} f(\hat{p}) \leq 0$. The weak axiom then implies $\hat{p}^{\prime} f(p)>0$. The Liapunov function is again $L(p)=(1 / 2)(p-\hat{p})^{\prime}(p-\hat{p})$

$$
\dot{L}(p)=(p-\hat{p}) / \hat{p}=\hat{p}^{\prime} f(p)+\hat{p}^{\prime} B(p)\left(B^{\prime}(p) B(p)\right)^{-1} B^{\prime}(p) f(p) .
$$

Notice that $y=\left(B^{\prime}(p) B(p)\right)^{-1} B^{\prime}(p) f(p)>0$ and $\hat{p}^{\prime} B(p) \leq 0$ since $p \in Y^{*}$. Consequently, $\dot{\mathrm{L}}(\mathrm{p})<0$ unless $\mathrm{p}=\hat{\mathrm{p}}$.

Van den Elzen, van der Laan, and Talman (1989) have generalized the path following method of van der Laan and Talman (1987) for computing equilibria of exchange economies to compute equilibria of economies with activity analysis production technologies. Their method, like 
the method for exchange economies, is similar to tatonnement, and, like the above method, always stays in the set of prices where $p^{\prime} A \leq 0$.

\subsection{Computation in the Space of Factor Prices}

The dimension of the space in which we must search for an equilibrium is usually the critical determinant of how difficult it is to find one. In production economies with a generalized input-output structure, where goods can be partitioned into produced goods and factors of production, we can reduce the dimension of the space to the number of factors. This reduction has often been used by applied general equilibrium modelers to improve computational efficiency (see, for example, Helpman 1976). It has also been used by international trade theorists in their discussions of factor-price equalization (see, for example, McKenzie 1955). Kehoe and Whalley (1985) exploit a reduction in dimension of this sort to carry out exhaustive searches to verify that two large-scale applied general equilibrium models have unique equilibria.

We begin with the activity analysis case. A production economy $(f, A)$ has a generalized input-output structure if it satisfies the following conditions:

- There are $h<n$ factors of production: $a_{i j} \leq 0$ for $i=1, \ldots, n$, and $j=1, \ldots, k$.

- There is no joint production: for every $\mathrm{j}=1, \ldots, \mathrm{k}, \mathrm{a}_{\mathrm{ij}}>0$ for at most one $\mathrm{i}$.

- Production of all produced goods is possible: there exists $y \geq 0$ such that $\sum_{j=1}^{m} a_{i j} y_{j}>0$, $\mathrm{i}=1, \ldots, \mathrm{n}-\mathrm{h}$.

- Every good that can be produced is actually produced at every equilibrium.

To ensure that this final condition holds, we could require that $f_{i}(p) \geq 0, i=1, \ldots, n-h$, for all p or, what is almost the same thing, that consumers have no endowments of produced goods. In the case where $h=1$ these conditions are those of the nonsubstitution theorem (see Samuelson 1951). 
Let $p \in R^{n-h}$ now be the vector of prices of produced goods and $q \in R^{h}$ be the vector of factor prices. Partition $A$ into

$$
\left[\begin{array}{l}
A_{1} \\
A_{2}
\end{array}\right]
$$

where $A_{1}$ is $(n-h) \times k$ and $A_{2}$ is $h \times k$. Similarly, partition $f(p, q)$ into $\left(f^{1}(p, q), f^{2}(p, q)\right.$ ).

In the case where $A$ consists of $2 n-h$ activities, $n$ disposal activities and an $n \times(n-h)$ matrix $B$ with one activity to produce each of the produced goods, the reduction of the search to the space of factor prices is easy. Our assumptions imply that the $(n-h) \times(n-h)$ matrix $B_{1}$ is a productive Leontief matrix. Under a mild indecomposibility assumption, $\mathrm{B}_{1}^{-1}$ is strictly positive. The equilibrium condition $B_{1} \hat{y}=f^{1}(\hat{q}, \hat{q})$ implies that $\hat{y}=B_{1}^{-1} f^{1}(\hat{p}, \hat{q})$, and the zero profit condition $\hat{p}^{\prime} B_{1}+q^{\prime} B_{2}=0$ implies that $\hat{p}=-\left(B_{2} B_{1}^{-1}\right)^{\prime} \hat{q}$. We are left with the equilibrium condition $B_{2} g=$ $f^{2}(p, q)$. We define the function $\psi: R_{+}^{h} \backslash\{0\} \rightarrow R^{h}$ by the rule

$$
\psi(q)=f^{2}\left(-\left(B_{2} B_{1}^{-1}\right)^{\prime} q, q\right)-B_{2} B^{-1} f^{1}\left(-\left(B_{2} B_{1}^{-1}\right)^{\prime} q, q\right) .
$$

It is easy to verify the $\psi$ is continuous, is homogeneous of degree zero, and obeys Walras's law because $f$ satisfies these properties. Notice that $\psi(q)=0$ is equivalent to

$$
\mathrm{f}^{2}(\hat{\mathrm{p}}, \hat{\mathrm{q}})=\mathrm{B}_{2} \mathrm{~B}_{1}^{-1} \mathrm{f}^{-1}(\hat{\mathrm{p}}, \hat{\mathrm{q}})=\mathrm{B}_{2} \hat{\mathrm{g}} \text {. }
$$

Consequently, $q$ is an equilibrium of the h-good exchange economy $\psi$ is and only if $(\hat{p}, \hat{q})$ is an equilibrium of the n-good production economy $(f, A)$.

When there is more than one possible activity for producing each good the situation is slightly more complicated. To calculate $\psi(\mathcal{Q})$ we start by solving the linear programming problem

$$
\min -\mathbf{q}^{\prime} \mathrm{A}_{2} \mathrm{Y}
$$

subject to

$$
A_{1} y=e
$$




\section{$y \geq 0$.}

Our assumptions imply that this problem is feasible and has a finite maximum. The nonsubstitution theorem says that the solution is associated with a feasible basis,

$$
\left[\begin{array}{c}
-q^{\prime} B_{2} \\
B_{1}
\end{array}\right] \text {, }
$$

that is, a matrix of $n-h$ columns associated with positive activity levels $y_{j}$ that does not vary as the right-hand side varies, although the activity levels themselves do. Furthermore, there is a vector of prices p such that $p^{\prime} B_{1}+q^{\prime} B_{2}=0$ and $p^{\prime} A_{1}+q^{\prime} A_{2} \leq 0$ (see Gale 1960, pp. 301-06). When the basis is uniquely defined, we can proceed as above.

There may, however, be more than one feasible basis possible in the solution. Although the linear programming problem may be degenerate, the economy itself need not be. Such is the case in Example 4.1, where the economy has this generalized input-output structure with $n=4$ and $h=2$. At two of its three equilibria, this example has $3>2=n-h$ activities in use. When there is more than one feasible basis possible, the demand for factors becomes a convex-valued, upper-hemi-continuous correspondence; $0 \in \psi(\hat{q})$ is then the equilibrium condition. (See Kehoe 1984 for details.)

With more general production technologies, the situation is similar to the activity analysis case with many activities. For any vector of factor prices $q$ we find the cost minimizing production plan for producing an arbitrary vector, say e, of produced goods. This plan is associated with a vector of prices $p(q)$, which can be plugged into the demand function $\mathrm{f}^{1}$ to find the production plan that satisfies the feasibility condition in the produced goods markets. This production plan induces a demand for factors of production. We systematically vary $q$ to make the excess demand for factors equal zero. 
The transformation of an economy with production into an exchange economy in factors is also useful for developing conditions sufficient for uniqueness of equilibrium. If $\psi$ satisfies the weak axiom of revealed preference or gross substitutability, for example, then there is a unique equilibrium. Mas-Colell (1989) uses this approach to show that an economy with a generalized input-output structure in which alt utility functions and production functions are Cobb-Douglas has a unique equilibrium because $\psi$ then exhibits gross substitutability. (He also reports that similar resuits have been obtained by Michael Jerison.) Mas-Colell (1989) further generalizes this to the assumption that utility and production functions are super-Cobb-Douglas in that they locally exhibit as much substitutability as a Cobb-Douglas function. The precise condition on $u: R_{+}^{\mathbf{n}} \rightarrow \mathbf{R}$, for example, is that for every $x \in R_{+}^{n}$ there exists a Cobb-Douglas function $u_{x}: R_{+}^{n} \rightarrow R$ and a neighborhood $U_{x}$ of $x$ such that $u_{x}(x)=u(x)$ and $u_{x}(z) \leq u_{x}(x)$ for all $z \in U_{x}$. For generalized input-output economies where $n-h=h=2$ and all utility and production functions are C.E.S., John (1989) has developed necessary and sufficient conditions for uniqueness of equilibrium.

\section{Economies with a Finite Number of Infinitely Lived Consumers}

We now consider economies in which goods are indexed by date and, possibly, state of nature. We assume that both time and uncertainty are discrete. If there are a finite number of time periods and a finite number of states of nature, then there is a finite number of goods, and this type of economy fits into the previous framework. Here, and in the next section, we consider economies with an infinite number of goods. Mas-Colell and Zame (1989) study questions related to existence of equilibrium in this type of economy in detail; here we focus on questions related to computation and multiplicity. 


\subsection{Determintstic Exchange Economies}

We begin by analyzing a deterministic stationary exchange economy with $m$ consumers and n goods in each period. Each consumer is specified by a utility function of the form $\sum_{t=1}^{\infty} \gamma_{i}^{t-1} u_{i}\left(x_{t}\right)$ and a vector of endowments that is the same in every period. Here $1>\gamma_{i}>0$ is a discount factor. We assume that $u_{i}: R_{+}^{\mathfrak{n}} \rightarrow R$ is strictly concave and monotonically increasing and that $w^{i}$ is strictly positive.

Let $p_{t}=\left(p_{1 t}, \ldots, p_{n t}\right)$ be the vector of prices in period $t$. An equilibrium of this economy is a price sequence $\hat{p}_{1}, \hat{p}_{2}, \ldots$, where $\hat{p}_{t} \in R_{+}^{\mathrm{a}} \backslash\{0\}$, and an allocation $\left(\hat{\mathrm{x}}^{1}, \ldots, \hat{\mathrm{x}}^{\mathrm{m}}\right), \hat{\mathrm{x}}^{i}=\left(\hat{\mathrm{x}}_{1}^{i}, \mathrm{x}_{2}^{i}, \ldots\right)$, where $\boldsymbol{x}_{\mathbf{t}}^{\mathbf{i}} \in \mathbf{R}_{+}^{\mathbf{n}}$,

- $\mathrm{x}_{1}^{\mathrm{i}}, \mathrm{x}_{2}^{\mathrm{i}}, \ldots$, solves

$$
\max \sum_{t=1}^{\infty} \gamma_{i}^{t-1} u_{i}\left(x_{t}\right)
$$

subject to

$$
\begin{aligned}
& \sum_{t=1}^{\infty} \hat{p}_{t}^{\prime} x_{t} \leq \sum_{t=1}^{\infty} p_{t}^{\prime} w^{i} \\
& x_{t} \geq 0 \\
& \text { - } \sum_{i=1}^{m} \hat{x}_{t}^{i} \leq \sum_{i=1}^{m} w^{i}, t=1,2, \ldots
\end{aligned}
$$

Using the Negishi (1960) approach, we characterize equilibria as solutions to social planning problems. This approach has been applied to dynamic economies by Bewley (1982) and Yano (1984).

Consider the Pareto problem

$$
\max \sum_{i=1}^{m} \alpha_{i} \sum_{t=1}^{\infty} \gamma_{i}^{t-1} u_{i}\left(x_{t}^{i}\right)
$$


subject to

$$
\begin{aligned}
& \sum_{i=1}^{m} x_{t}^{i} \leq \sum_{i=1}^{m} w^{i}, \quad t=1,2, \ldots \\
& x_{t}^{i} \geq 0
\end{aligned}
$$

for a vector $\alpha$ of strictly positive welfare weights. Using the same reasoning as before, we can argue that, if $\alpha_{i}=1 \hat{\lambda}_{i}$ where $\hat{\lambda}_{i}$ is the equilibrium value of the Lagrange multiplier for the budget constraint of consumer $i$, then the competitive allocation $\left(\mathrm{x}^{1}, \ldots, \mathrm{x}^{\mathrm{m}}\right)$ solves this problem where $\mathrm{p}_{\mathrm{t}}(\alpha)=\mathrm{p}_{\mathrm{t}}$ is the vector of Lagrange multipliers for the feasibility constraint in period t. Consequently, the first welfare theorem holds for this economy.

The crucial step in the reasoning is taking the necessary and sufficient condition for the solution to the consumer's maximization problem,

$$
\begin{gathered}
\sum_{t=1}^{\infty} \gamma_{i}^{t-1} u_{i}\left(\hat{x}_{t}^{i}\right)+\lambda_{i} \sum_{t=1}^{\infty} \hat{p}_{t}^{\prime}\left(w^{i}-x_{t}^{i}\right) \geq \sum_{t=1}^{\infty} \gamma_{i}^{t-1} u_{i}\left(x_{t}^{i}\right)+\hat{\lambda}_{i} \sum_{t=1}^{\infty} \hat{p}_{t}^{\prime}\left(w^{i}-x_{t}^{i}\right) \\
\geq \sum_{t=1}^{\infty} \gamma_{i}^{t-1} u_{i}\left(x_{t}^{i}\right)+\lambda_{i} \sum_{t=1}^{\infty} \hat{p}_{t}^{\prime}\left(w^{i}-x_{t}^{i}\right)
\end{gathered}
$$

for all $\lambda_{i} \geq 0$ and $x_{t}^{i} \geq 0$, dividing through by $\hat{\lambda}_{i}>0$, and summing over consumers to produce

$$
\begin{aligned}
& \sum_{i=1}^{m}\left(1 \hat{\lambda}_{i}\right) \sum_{t=1}^{\infty} \gamma_{i}^{t-1} u_{i}\left(\hat{x}_{t}^{i}\right)+\sum_{t=1}^{\infty} \hat{\rho}_{t}^{\prime} \sum_{i=1}^{m}\left(w^{i}-\hat{x}_{t}^{i}\right) \\
& \geq \sum_{i=1}^{m}\left(1 / \hat{\lambda}_{i}\right) \sum_{t=1}^{\infty} \gamma_{i}^{t-1} u_{i}\left(x_{t}^{i}\right)+\sum_{t=1}^{\infty} \hat{p}_{t}^{i} \sum_{i=1}^{m}\left(w^{i}-x_{t}^{i}\right)
\end{aligned}
$$

for all $x_{t}^{i} \geq 0$. Since $\sum_{t=1}^{\infty} \hat{p}_{t}^{\prime} w^{i}$ must be finite for the consumer's utility maximization problem to have a solution, $\sum_{i=1}^{m} \sum_{t=1}^{\infty} \hat{p}_{t}^{\prime} w^{i}$ must also be finite. 
The second welfare theorem holds for this economy as well. The transfer payments needed to decentralize a solution to the Pareto problem $\left(\mathrm{x}^{1}(\alpha), \ldots, \mathrm{x}^{\mathrm{m}}(\alpha)\right)$ as an equilibrium with transfer payments are

$$
t_{i}(\alpha)=\sum_{t=1}^{\infty} p_{t}(\alpha)^{\prime}\left(x_{t}^{i}(\alpha)-w^{i}\right), \quad i=1, \ldots, m
$$

Once again we can argue that $t$ is a convex-valued, bounded, upper-hemi-continuous correspondence. Consequently, Proposition 3.2 applies, and there exists a vector of welfare weights $\hat{\alpha}$ such that $\left(\mathrm{p}(\hat{\alpha}),\left(\mathrm{x}^{1}(\hat{\alpha}), \ldots, \mathrm{x}^{\mathrm{m}}(\hat{\alpha})\right)\right)$ is an equilibrium $0 \in \mathrm{t}(\hat{\alpha})$.

It is possible to extend our analysis to the more general stationary preferences described by Koopmans, Diamond, and Williamson (1964). This extension is most easily done using the dynamic programming framework described in the next section.

Example 5.1. Consider a simple economy with one good in each period and two consumers. Suppose that $u_{1}\left(x_{t}\right)=u_{2}\left(x_{t}\right)=\log x_{t}$ and that $w^{1}=w^{2}=1$. The only difference between the two consumers is that $\gamma_{1}<\gamma_{2}$. A solution to the utility maximization is characterized by the conditions

$$
\begin{aligned}
& \gamma_{i}^{t-1} / x_{t}^{i}=\lambda_{i} p_{t} \\
& \sum_{t=1}^{\infty} p_{t} x_{t}^{i}=\sum_{t=1}^{\infty} p_{t} .
\end{aligned}
$$

An equilibrium satisfies these conditions and the condition that demand equals supply:

$$
c_{t}^{1}+c_{t}^{2}=2, \quad t=1,2, \ldots
$$

The Pareto problem is

$$
\max \alpha_{1} \sum_{t=1}^{\infty} \gamma_{1}^{t-1} \log x_{t}^{1}+\alpha_{2} \sum_{t=1}^{\infty} \gamma_{2}^{t-1} \log x_{t}^{2}
$$


subject to

$$
x_{t}^{1}+x_{t}^{2}=2, \quad t=1,2, \ldots
$$

A solution to this problem is characterized by the conditions

$$
\alpha_{i} \gamma_{i}^{t-1} / x_{t}^{i}=p_{t}, \quad i=1,2,
$$

and the feasibility conditions. These equations can easily be solved to yield

$$
\begin{aligned}
& x_{t}^{i}=\frac{2 \alpha_{i} \gamma_{i}^{t-1}}{\alpha_{1} \gamma_{1}^{t-1}+\alpha_{2} \gamma_{2}^{t-1}}, \quad i=1,2 . \\
& p_{t}=\left(\alpha_{1} \gamma_{1}^{t-1}+\alpha_{2} \gamma_{2}^{t-1}\right) / 2 .
\end{aligned}
$$

The transfer payments needed to implement as a competitive equilibrium the allocation associated with the weights $\alpha_{1}$ and $\alpha_{2}$ are, therefore,

$$
\begin{aligned}
& t_{1}\left(\alpha_{1}, \alpha_{2}\right)=\sum_{t=1}^{\infty} p_{t}\left(x_{t}^{1}-1\right)=\frac{\alpha_{1}}{1-\gamma_{1}}-\frac{\alpha_{2}}{1-\gamma_{2}} \\
& t_{2}\left(\alpha_{1}, \alpha_{2}\right)=\sum_{t=1}^{\infty} p_{t}\left(x_{t}^{2}-1\right)=\frac{\alpha_{2}}{1-\gamma_{2}}-\frac{\alpha_{1}}{1-\gamma_{1}}
\end{aligned}
$$

Notice that these functions are continuously differentiable, are homogeneous of degree one, and sum to zero. The unique equilibrium is found by setting these transfer payments equal to zero. It is $\left(\hat{\alpha}_{1}, \hat{\alpha}_{2}\right)=\left(\left(1-\gamma_{1}\right) /\left(2-\gamma_{1}-\gamma_{2}\right),\left(1-\gamma_{2}\right) /\left(2-\gamma_{1}-\gamma_{2}\right)\right)$

\subsection{Dynamic Production Economies and Dynamic Programming}

It is possible to analyze economies with production and durable goods in much the same way as we have just done with exchange economies. Frequently, however, it is convenient, both for computing equilibria and for studying their properties, to recast the problem of determining an equilibrium of a dynamic economy with production as a dynamic programming problem. 
Consider an economy with $m$ infinitely lived consumers and $n_{c}$ perishable consumption goods. Each consumer has a utility function $\sum_{t=1}^{\infty} \gamma^{t-1} u_{i}\left(x_{t}\right)$. Here $u_{i}$ is strictly concave and monotonically increasing and $\gamma$ is a discount factor common to all consumers; we tater discuss heterogeneous discount factors. The endowment of these goods held by consumer $i$ in each period is $w^{i} \in R_{+}^{\mathbf{n}_{\mathrm{c}}}$; it may include different types of labor. In addition, there are $n_{k}$ capital goods, reproducible factors of production. The endowment of these goods held by consumer $i$ in the first period is $k_{1}^{i} \in R_{+}^{n_{k}}$.

There are many ways to specify the production technology. One of the easiest is to define an aggregate production function $h: R_{+}^{n_{k}} \times R_{+}^{n_{k}} \times R^{n_{c}} \rightarrow R$ that is continuous, concave, homogeneous of degree one, increasing in the first vector of arguments, and decreasing in the second and third. A feasible production-consumption plan in period $t$ is then a triple that satisfies

$$
h\left[k_{t}, k_{t+1}, \sum_{i=1}^{m}\left(x_{t}^{i}-w\right)\right] \geq 0 .
$$

Here $k_{t}$ is the input of capital goods, $k_{t+1}$ is the output of capital goods that can be used in the next period, and $\sum_{i=1}^{m}\left(x_{t}^{i}-w^{i}\right)$ is the net output of consumption goods. To keep the equilibrium path of capital stocks bounded, we assume that there exists a vector $k^{\max } \in R_{+}^{n_{k}}$ such that if $\left\|k_{t}\right\| \geq$ $\left\|k^{\max }\right\|$ and $\left\|k_{t+1}\right\| \geq\left\|k_{t}\right\|$, where $k_{t}, k_{t+1} \in R_{+}^{n_{k}}$ then $h\left(k_{t}, k_{t+1}, z\right)<0$ for all $z \geq-\sum_{i=1}^{n} w^{i}$; in other words, it is not feasible to sustain a path of capital stocks with $\left\|k_{t}\right\| \geq$ $\left\|\mathrm{k}^{\max }\right\|$.

A simple example of such a function $h$ is

$$
h\left(k_{t}, k_{t+1}, z_{1 t}, z_{2 t}\right)=\eta k_{t}^{\theta}\left(-z_{1}\right)^{1-\theta}+(1-\delta) k_{t}-k_{t+1}-z_{2 t} \text {. }
$$

Here there is a single capital good, $k_{t}$, and two consumption goods, leisure, $z_{1 t}$, and consumption $z_{2 t}$. The feasibility constraint says that consumption and investment net of depreciation must be less or equal to than the output of a Cobb-Douglas production function. 
An equilibrium of this economy is a sequence of prices for the consumption goods $p_{1}, \hat{p}_{2}$, $\ldots$, where $\hat{p}_{t} \in R_{+}^{n_{c}} \backslash\{0\}$; a price $t \in R_{+}^{n_{k}}$ for the initial capital stock; an allocation, $x_{1}^{i}, x_{2}^{i}, \ldots$, where $x_{t}^{i} \in R_{+}^{n_{c}}$ for each consumer $i$; a sequence of net outputs of consumption goods $z_{1}, z_{2}, \ldots$, where $\hat{z}_{t} \in R^{n_{c}}$; and a sequence of capital goods $\hat{k}_{1}, \hat{k}_{2}, \ldots$, where $\hat{k}_{t} \in R_{+}^{n_{k}}$ such that

- $\mathbf{x}_{1}^{\mathrm{i}}, \mathrm{x}_{2}^{\mathrm{i}}, \ldots$ solves

$$
\max \sum_{t=1}^{\infty} \gamma^{t-1} u_{i}\left(x_{t}\right)
$$

subject to

$$
\begin{aligned}
& \sum_{t=1}^{\infty} \hat{p}_{t}^{\prime} x_{t} \leq \sum_{t=1}^{\infty} \hat{p}_{t}^{\prime} w^{i}+p^{\prime} k_{l}^{i} \\
& x_{t} \geq 0 . \\
& -t_{1}, z_{2}, \ldots, \text { and } \hat{k}_{1}, \hat{k}_{2}, \ldots \text { solve } \\
& \max _{t=1}^{\infty} \hat{p}_{t z_{t}}^{\prime}-\hat{p}^{\prime} k_{1}
\end{aligned}
$$

subject to

$$
\begin{aligned}
& h\left(k_{t}, k_{t+1}, z_{t}\right) \geq 0, \quad t=1,2, \ldots \\
& k_{t} \geq 0 . \\
& \text { - } \sum_{i=1}^{m} x_{t}^{i}=z_{t}+\sum_{i=1}^{m} w^{i}, \quad t=1,2, \ldots \\
& \text { - } \sum_{i=1}^{m} k_{1}^{i}=k_{1} .
\end{aligned}
$$

Again, an equilibrium is Pareto efficient and solves a Pareto problem of the form

$$
\max \sum_{i=1}^{m} \alpha_{i} \sum_{t=1}^{\infty} \gamma^{t-1} u_{i}\left(x_{i}^{i}\right)
$$


subject to

$$
\begin{aligned}
& h\left[k_{t}, k_{t+1}, \sum_{i=1}^{m}\left(x_{t}^{i}-w^{i}\right)\right] \geq 0, \quad t=1,2, \ldots \\
& k_{1}=\sum_{i=1}^{m} k_{1}^{i} \\
& x_{t}^{i}, \quad k_{t} \geq 0
\end{aligned}
$$

for an appropriate vector $\alpha$ of strictly positive welfare weights.

In principle, we have all that we need to characterize equilibria of this economy: using the Lagrange multipliers $\pi_{t}$ for this problem and the derivatives of $h$, or subgradients if $h$ is not continuously differentiable, we can calculate

$$
\begin{aligned}
& p_{t}(\alpha)=-\pi_{t}(\alpha) D_{3} h\left[k_{t}(\alpha), k_{t+1}(\alpha), \sum_{i=1}^{m}\left(x_{t}^{i}(\alpha)-w^{i}\right)\right], \quad t=1,2, \ldots \\
& r(\alpha)=\pi_{1}(\alpha) D_{1} h\left[\sum_{i=1}^{m} k_{1}^{i}, k_{2}(\alpha), \sum_{i=1}^{m}\left(x_{1}^{i}(\alpha)-w^{i}\right)\right] \\
& t_{i}(\alpha)=\sum_{t=1}^{\infty} p_{t}(\alpha)^{\prime} p_{t}(\alpha)^{\prime}\left(x_{t}^{i}(\alpha)-w^{i}\right)-r(\alpha)^{\prime} k_{i}^{i}, \quad i=1, \ldots, m .
\end{aligned}
$$

Once again, equilibria are solutions to the equation $t(\alpha)=0$.

We can greatly simplify the characterization of solutions to the Pareto problem, and computation equilibria, however, using the theory of dynamic programming. (See Bellman 1957; Blackwell 1965; Hansen and Prescott 1991; Harris 1987; Sargent 1987; and Stokey, Lucas, and Prescott 1989 provide useful summaries and economic applications.) Given an aggregate endowment of capital in the first period $k_{1} \in \mathbf{R}_{+}^{\mathbf{n}_{k}}$, an aggregate endowment of consumption goods in each period $w \in R_{+}^{n_{c}}$, and welfare weights $\alpha \in R_{+}^{m_{+}} \backslash\{0\}$, we define the value function $V\left(k_{1}, w, \alpha\right)$ as 


$$
\max \sum_{i=1}^{m} \alpha_{i} \sum_{t=1}^{\infty} \gamma^{t-1} u_{i}\left(x_{t}^{i}\right)
$$

subject to

$$
\begin{aligned}
& h\left[k_{t}, k_{t+1}, \sum_{i=1}^{m}\left(x_{t}^{i}-w\right)\right] \geq 0, \quad t=1,2, \ldots \\
& x_{t}^{i}, \quad k_{t} \geq 0 .
\end{aligned}
$$

It is easy to show that $V$ is continuous, concave in $k_{1}$ and $w$ and convex in $\alpha$. If it is continuously differentiable, the envelope theorem allows us to use $D_{1} V\left(k_{1}, w, \alpha\right)$ as a price vector of capital, $r^{\prime}$, and $D_{2} V\left(k_{1}, w, \alpha\right)$ as a price vector for the present value of the endowment of consumption goods, $\sum_{\mathrm{t}=1}^{\infty} \mathrm{p}_{\mathrm{t}}^{\prime}$. If $\mathrm{V}$ is not continuously differentiable, we can work with subgradients. (Benviniste and Scheinkman 1979 provide general conditions that ensure the $V$ is continuously differentiable.) The value of the total endowment of consumer $i$ is

$$
D_{1} v\left[\sum_{j=1}^{m} k_{1}^{j}, \sum_{j=1}^{m} w^{j}, \alpha\right] k_{1}^{i}+D_{2} v\left[\sum_{j=1}^{m} k_{i}^{i}, \sum_{j=1}^{m} w^{j}, \alpha\right] w^{i}
$$

To calculate the transfer functions $t_{i}(\alpha)$, we need to calculate the value of expenditures. To do this in terms of the value function, we introduce $m$ consumer specific goods and define $U_{i}$ : $\mathbf{R}_{+}^{\mathbf{n}_{\mathrm{c}}} \times \mathbf{R}_{++}^{\mathbf{m}} \rightarrow \mathrm{R}$ as $\mathrm{U}_{\mathrm{i}}(\mathrm{x}, \mathrm{y})=\mathrm{y}_{\mathrm{i}} \mathrm{u}_{\mathrm{i}}\left(\mathrm{x} / \mathrm{y}_{\mathrm{i}}\right)$. Notice that $\mathrm{U}_{\mathrm{i}}$ is homogeneous of degree one and that $\mathrm{U}_{\mathrm{i}}(\mathrm{x}, \mathrm{e})=\mathrm{u}_{\mathrm{i}}(\mathrm{x})$ where $\mathrm{e}=(1, \ldots, 1)$. We endow consumer $\mathrm{i}$ with the entire aggregate endowment of one unit of $y_{i}$. We now define $V\left(k_{1}, w, y, \alpha\right)$ as the maximum of weighted sum of the augmented utility functions subject to the feasibility constraints. If $x_{t}^{i}$ is the optimal consumption of consumer $i$ in period $t$, its value is

$$
\gamma^{t-1} \alpha_{i} D u_{i}\left(x_{t}^{i}\right) x_{t}^{i}=\gamma^{t-1} \alpha_{i} D_{i} U_{i}\left(x_{t}^{i}, e\right) x_{i}^{i}
$$


Since $U_{i}$ is homogeneous of degree one, we know that

$$
D_{1} U_{i}\left(x_{t}^{i}, e\right) x_{t}^{i}=U_{i}\left(x_{t}^{i}, e\right)-D_{2} U_{i}\left(x_{t}^{i}, e\right) e .
$$

Using the envelope theorem, we can show that

$$
\sum_{t=1}^{\infty} \gamma^{t-1} \alpha_{i} U_{j}\left(x_{t}^{i}, e\right)=\alpha_{i} \partial V\left(\sum_{i=1}^{m} k_{1}^{i}, \sum_{i=1}^{m} w^{i}, e, \alpha\right] / \partial \alpha_{i}
$$

and that

$$
\sum_{t=1}^{\infty} \gamma^{t-1} \alpha_{i} D_{2} U_{i}\left(x_{t}^{i}, e\right) e=\partial V\left(\sum_{i=1}^{m} k_{1}^{i}, \sum_{i=1}^{m} w^{i}, e, \alpha\right) / \partial y_{i}
$$

Consequently, the value of the expenditures of consumer $i$ is

$$
\begin{aligned}
\sum_{t=1}^{\infty} \gamma^{t-1} \alpha_{i} D_{1} U_{i}\left(x_{t}^{i}, e\right) x_{t}^{i}= & \alpha_{i} \partial V\left(\sum_{i=1}^{m} k_{1}^{i}, \sum_{i=1}^{m} w^{i}, e, \alpha\right] / \partial \alpha_{i} \\
& -\partial V\left(\sum_{i=1}^{m} k_{1}^{i}, \sum_{i=1}^{m} w^{i}, e, \alpha\right] / \partial y_{i} .
\end{aligned}
$$

We can now define the transfer function $t: R_{+}^{m} \backslash\{0\} \rightarrow R^{m}$ as

$$
\begin{aligned}
t_{i}(\alpha)= & \alpha_{i} \partial V\left(\sum_{j=1}^{m} k_{1}^{j}, \sum_{j=1}^{m} w^{j}, e, \alpha\right] / \partial \alpha_{i}-\partial V\left(\sum_{j=1}^{m} k_{1}^{j}, \sum_{j=1}^{m} w^{j}, e, \alpha\right) / \partial y_{i} \\
& -D_{1} V\left(\sum_{j=1}^{m} k_{1}^{i}, \sum_{j=1}^{m} w^{i}, e, \alpha\right] k_{1}^{i}-D_{2} V\left(\sum_{j=1}^{m} k_{l}^{j}, \sum_{j=1}^{m} w^{j}, e, \alpha\right)
\end{aligned}
$$

If $\mathrm{V}$ is continuously differentiable, $\mathrm{t}$ is a continuous function. If $\mathrm{V}$ is not continuously differentiable, $t$ is a nonempty, upper-hemi-continuous, convex-valued correspondence. Once again, $t$ is homogeneous of degree one and satisfies the identity 


$$
\sum_{i=1}^{m} t_{j}(\alpha)=0
$$

(See Kehoe, Levine, and Romer 1989b, 1990 for details.) Furthermore, if $\alpha \in R_{+}^{m} \backslash\{0\}$ and $\alpha_{i}=0$, then $t_{i}(\alpha)<0$. The same argument as in the proof of Proposition 3.2 now implies the existence of equilibrium.

Using dynamic programming theory, we can investigate the properties of $\mathrm{V}$ and, therefore, of $t$. We define the return function $v\left(k_{t}, k_{t+1}, w, y, \alpha\right)$ as

$$
\max \sum_{i=1}^{m} \alpha_{i} U_{i}\left(x^{i}, y\right)
$$

subject to

$$
\begin{aligned}
& h\left[k_{t}, k_{t+1}, \sum_{i=1}^{m} x^{i}-w\right) \geq 0 \\
& x^{i} \geq 0
\end{aligned}
$$

If $h\left(k_{t}, k_{t+1},-w\right)<0$, we let $v\left(k_{t}, k_{t+1}, w, y, \alpha\right)=-\infty$. The function $v$ is upper-semi-continuous on the extended real line $R \cup\{-\infty\}$; see Kehoe, Levine, and Romer (1989b, 1990). The value function $\mathrm{V}$ can be found by choosing $k_{2}, k_{3}, \ldots$ to solve

$$
\max \sum_{t=1}^{\infty} \gamma^{t-1} v\left(k_{t}, k_{t+1}, w, y, \alpha\right)
$$

subject to

$$
\mathbf{k}_{\mathbf{t}} \geq 0
$$

The direct approach to this problem focuses on the first order conditions, often referred to as Euler equations,

$$
\gamma D_{1} v\left(k_{t}, k_{t+1}, w, y, \alpha\right)+D_{2} v\left(k_{t-1}, k_{t}, w, y, \alpha\right)=0 .
$$


This is a system of $n_{k}$ second-order difference equations in the state variables $k_{t *}$ There are, however, only $n_{k}$ initial conditions, the fixed values of $k_{1}$. For some choices of $k_{2}$ the corresponding path $k_{1}, k_{2}, k_{3}, \ldots$ eventually leads to negative capital stocks and the system breaks down. For others, we need additional boundary conditions to single out the unique path that is optimal. These are given by the transversality conditions

$$
\lim _{t \rightarrow \infty} \gamma^{t-1} D_{1} v\left(k_{t}, k_{t+1}\right) k_{t}=0
$$

Showing that the transversality conditions together with the Euler equations are sufficient for optimality is relatively easy; see, for example, Stokey, Lucas, and Prescott (1989, pp. 97-99). Under additional assumptions, they can also be shown to be necessary; see Peleg and Ryder (1972), Weitzman (1973), and Ekeland and Scheinkman (1986).

An alternative approach to finding the value function involves solving the functional equation

$$
\mathrm{V}\left(\mathrm{k}_{\mathrm{t}}, \mathrm{w}, \mathrm{y}, \alpha\right)=\max \left[\mathrm{v}\left(\mathrm{k}_{\mathrm{t}}, \mathrm{k}_{\mathrm{t}+1}, \mathrm{w}, \mathrm{y}, \alpha\right)+\gamma \mathrm{V}\left(\mathrm{k}_{\mathrm{t}+1}, \mathrm{w}, \mathrm{y}, \alpha\right)\right]
$$

subject to

$$
k_{t+1} \geq 0
$$

for the function $V$. The vector $k_{t+1}=g\left(k_{t}, w, y, \alpha\right)$ that solves this problem is referred to as the policy function. Calculating optimal paths is much easier using the policy function than it is using the Euler equations: we simply run the $n_{k}$ first order difference equations

$$
k_{t+1}=g\left(k_{t}, \sum_{i=1}^{m} w^{i}, e, \alpha\right)
$$

forward from $k_{1}$ without worrying about the transversality conditions. The difficulty is in finding the policy function $g$. Keep in mind that, whether we use the Euler equation approach or this second, the dynamic programming approach, we must somehow calculate the equilibrium values of $\alpha$. 
Example 5.2. Consider an economy with two consumption goods, leisure and consumption, and one capital good. There is a representative consumer with utility

$$
\sum_{t=1}^{\infty} \gamma^{t-1} u\left(\ell_{t}, c_{t}\right)=\sum_{t=1}^{\infty} \gamma^{t-1} \log c_{t}
$$

(This function does not actually satisfy our assumptions because $u$ is not strictly concave in $\ell_{\mathrm{t}}$ and it is not continuous at $c_{t}=0$; this is not essential, however.) The endowment is $(w, 0)$ of labor and consumption every period and $\mathbf{k}_{1}$ of capital in the first period. The production function is

$$
h\left(k_{t}, k_{t+1}, z_{1 t}, z_{2 t}\right)=\eta k_{t}^{\theta}\left(-z_{1 t}\right)^{1-\theta}-k_{t+1}-z_{2 t} .
$$

The return function is

$$
\mathrm{v}\left(\mathrm{k}_{\mathrm{t}}, \mathrm{k}_{\mathrm{t}+1}, \mathrm{w}, \mathrm{y}, \alpha\right)=\alpha \mathrm{y} \log \left(\eta \mathrm{k}_{\mathrm{t}}^{\theta} \mathrm{w}^{1-\theta}-\mathrm{k}_{\mathrm{t}+1}\right)-\alpha y \log \mathrm{y} .
$$

This return function is special in that we can analytically solve for the value function $V\left(k_{t}, w, y, \alpha\right)$. This example is a member of a special class of examples first studed by Radner (1966). Notice that we do not allow the per period endowment of consumption to vary from zero; if we do, we cannot solve for the value function analytically.

The value function has the form $a_{1}+a_{2} \log k_{t}$. Using the functional relation

$$
a_{1}+a_{2} \log k_{t}=\max \left[\alpha y \log \left(\eta k_{t}^{\theta} w^{1-\theta}-k_{t+1}\right)-\alpha y \log y+\gamma\left(a_{1}+a_{2} \log k_{t+1}\right)\right]
$$

subject to

$$
k_{t+1} \geq 0 \text {, }
$$

we first solve for $k_{t+1}$, then substitute in the results and solve for $a_{1}$ and $a_{2}$. We obtain

$$
\begin{aligned}
\mathrm{V}\left(\mathrm{k}_{\mathrm{t}}, \mathrm{w}, \mathrm{y}, \alpha\right)=\frac{\alpha \mathrm{y}}{1-\gamma}[\log \eta(1-\gamma \theta) & +\frac{\gamma \theta}{1-\gamma \theta}+\log \gamma \eta \theta-\log \mathrm{y} \\
& \left.+\frac{1-\theta}{1-\gamma^{\theta}} \log w+\frac{\theta-\gamma \theta}{1-\gamma \theta} \log \mathrm{k}_{\mathrm{t}}\right] .
\end{aligned}
$$


The policy function is

$$
\mathrm{g}\left(\mathrm{k}_{\mathrm{t}}, \mathrm{w}, \mathrm{y}, \alpha\right)=\gamma \theta \eta \mathrm{w}^{1-\theta} \mathrm{k}_{\mathrm{t}}^{\theta}
$$

Notice that, for any initial value $k_{1}$, the sequence $k_{1}, k_{2}, \ldots$, converges to the steady state $\overline{\mathrm{k}}=$ $(\gamma \theta \eta)^{1 / 1-\theta_{\mathrm{w}}}$. To see this, consider

$$
\log k_{t+1}=\log \gamma \theta \eta w^{1-\theta}+\theta \log k_{t} .
$$

Since $0<\theta<1, \log \mathrm{k}_{\mathrm{t}}$ converges to $\left(\log \gamma \theta\right.$ n $\left.w^{1-\theta}\right) /(1-\theta)$.

The Euler equation is

$$
\frac{\gamma \theta \eta \mathrm{w}^{1-\theta} \mathrm{k}_{\mathrm{t}}^{\theta-1}}{\eta \mathrm{w}^{1-\theta} \mathrm{k}_{\mathrm{t}}^{\theta}-\mathrm{k}_{\mathrm{t}+1}}-\frac{1}{\eta \mathrm{w}^{1-\theta} \mathrm{k}_{\mathrm{t}-1}^{\theta}-\mathrm{k}_{\mathrm{t}}}=0, \quad \mathrm{t}=2,3, \ldots
$$

We know the initial value $k_{1}$, but we need a value for $k_{2}$ to get started. If we use any value other than $\mathrm{k}_{2}=\gamma \theta \eta \mathrm{w}^{1-\theta} \mathrm{k}_{1}^{\theta}$, however, we eventually have a negative capital stock, at which point the difference equation breaks down, or we violate the transversality condition. (To see this, rewrite the Euler equation as a first-order linear difference equation in $K_{t}=k_{t+1} k_{t}^{-\theta}$.)

Since this example has a representative consumer, the transfer function is identically equal to zero. Although the transfer function itself is not particularly interesting, we can use the value function $V\left(k_{t}, w, y, \alpha\right)$ to find the equilibrium value of the endowment

$$
\begin{aligned}
& \mathrm{r}=\mathrm{D}_{1} \mathrm{~V}\left(\mathrm{k}_{1}, \mathrm{w}, 1,1\right)=\theta /\left[(1-\gamma \theta) \mathrm{k}_{1}\right] \\
& \sum_{\mathrm{t}=1}^{\infty} \mathrm{p}_{1 \mathrm{t}}=\mathrm{D}_{2} \mathrm{~V}\left(\mathrm{k}_{1}, \mathrm{w}, 1,1,\right)=(1-\theta) /[(1-\gamma)(1-\gamma \theta) \mathrm{w}] .
\end{aligned}
$$

Consequently,

$$
\mathrm{rk}_{1}+\sum_{\mathrm{t}=1}^{\infty} \mathrm{p}_{1 \mathrm{t}} \mathrm{w}=1 /(1-\gamma)
$$

is both the value of the endowment and the value of expenditures. 
Remark. This example is a special case of the model developed by Brock and Mirman (1972). Long and Plosser (1983) work out a multisector version of a model with the same functional forms that permit an analytical solution.

Many extensions of this sort of analysis are possible. One is to economies with more general stationary preferences of the sort described by Koopmans, Diamond, and Williamson (1964). Letting $t^{c}=\left(c_{t}, c_{t+1}, \ldots\right)$, these preferences satisfy the functional relation

$$
\mathrm{U}(\mathrm{c})=\mathrm{W}\left(\mathrm{c}_{\mathrm{t}}, \mathrm{U}\left(\mathrm{t}+1^{\mathrm{c}}\right)\right)
$$

for an aggregator function $\mathrm{W}: \mathrm{R}_{+}^{\mathrm{n}_{\mathrm{c}}} \times \mathrm{R} \rightarrow \mathrm{R}$ that satisfies certain properties. (See, for example, Lucas and Stokey 1984, Dana and LeVan 1987, and Streufert 1990.)

Another possible extension is to retain additively separable utility functions but allow heterogeneous discount factors. One approach is to allow the return function $v\left(k_{t}, k_{t+1}, w, y, \alpha, t\right)$ to vary over time as the solution to maximizing $\sum_{\mathrm{i}=1}^{\mathrm{m}} \alpha_{\mathrm{i}}\left(\beta_{\mathrm{i}} / \beta_{1}\right)^{\mathrm{t}} \mathrm{U}_{\mathrm{i}}\left(\mathrm{x}^{\mathrm{i}}, \mathrm{y}\right)$ subject to feasibility constraints; here $\beta_{1}$ is the largest discount factor. (See Coles 1985.)

Yet another extension is to allow $k_{t}$ and $x_{t}$ to grow without bound. This is particularly useful in growth models of the sort considered by Romer (1986) and Lucas (1988). For an example that fits clearly into the competitive framework used here, see Jones and Manuelli (1990). In this sort of model care must be taken to ensure that $x_{t}$ cannot grow so fast that the present value of utility can become infinitely large.

\subsection{Stochastic Economies}

The analysis of the previous section can be easily modified to include random events. To do so, we follow the approach of Arrow (1953) and Debreu (1959) in indexing goods by both date and state of nature. Suppose that in period t one of a finite number of events $\eta_{t}=1, \ldots, \ell$ can occur. A state is a history of events $s_{t}=\left(\eta_{1}, \ldots, \eta_{t}\right)$, a node on the event tree. The event $\eta_{t}$ can 
effect preferences, endowments, or technology. Since the set of date-state pairs is countable, the analysis of Section 5.1 can be applied to prove the existence of equilibrium. We can again reduce the problem of computing an equilibrium to finding a set of welfare weights for which the corresponding Pareto efficient allocation can be decentralized as a transfer equilibrium with all transfers equal to zero.

Suppose that the probability of event $i$ is $\pi_{i}>0$ where $\sum_{j}^{\ell}=1 \pi_{j}=1$. The induced probability distribution even states is given by $\pi\left(s_{t}\right)=\pi_{\eta_{1}} \ldots \pi_{\eta_{t}}$. Let $S$ be the set of all possible states; let $t(s)$ be the date in which s occurs, that is, the length of the history $s$; let $s_{-1}$ be the history of length $t(s)-1$ that coincides with $s$; and let $\eta_{s}=\eta_{t(s)}$, that is, the last event in the history $s$. Assuming that consumers maximize expected utility, we can write the Pareto problem as

$$
\max \sum_{i=1}^{m} \alpha_{i} \sum_{s \in S} \gamma^{t(s)-1} \pi(s) u_{i}\left(x^{i}(s), \eta_{s}\right)
$$

subject to

$$
\begin{aligned}
& h\left[k\left(s_{-1}\right), k(s), \sum_{i=1}^{m}\left(x^{i}(s)-w^{i}\left(\eta_{s}\right), \eta_{s}\right] \geq 0, \quad s \in S\right. \\
& k_{1} \leq \sum_{i=1}^{m} k_{1}^{i} \\
& x^{i}(s), \quad k(s) \geq 0 .
\end{aligned}
$$

Here $k_{1}$ is the amount of capital before the event in the first period occurs.

As before, we define the return function $v\left(k_{t}, k_{t+1}, \eta_{t}, w, y, \alpha\right)$ as

$$
\max \sum_{i=1}^{m} \alpha_{i} U_{i}\left(x^{i}, y, \eta_{t}\right)
$$

subject to 


$$
\begin{aligned}
& h\left[k_{t}, k_{t+1}, \sum_{i=1}^{m} x^{i}-w\left(\eta_{t}\right), \eta_{t}\right] \geq 0 \\
& x^{i} \geq 0 .
\end{aligned}
$$

(Notice that now $w$ is a vector of dimension $n_{c} \times \ell$. ) The value function $v\left(k_{1}, \eta_{1}, w, y, \alpha\right)$ can be found by choosing $k(s), s \in S$, to solve

$$
\max \sum_{s \in S} \gamma^{t(s)-1} \pi(s) v\left(k\left(s_{-1}\right), k(s), \eta_{s}, w, y, \alpha\right)
$$

subject to

$$
k(s) \geq 0 \text {. }
$$

Once again we can characterize solutions to the Pareto problem using either the Euler equations

$$
\mathrm{D}_{2} \mathrm{v}\left(\mathrm{k}_{\mathrm{t}-1}, \mathrm{k}_{\mathrm{t}}, \eta_{\mathrm{t}-1}, \mathrm{w}, \mathrm{y}, \alpha\right)+\gamma \sum_{\eta=1}^{\ell} \pi_{\eta} \mathrm{D}_{1} \mathrm{v}\left(\mathrm{k}_{\mathrm{t}}, \mathrm{k}_{\mathrm{t}+1}, \eta, \mathrm{w}, \mathrm{y}, \alpha\right)=0
$$

and an expected value version of the transversality condition or using the functional equation

$$
\mathrm{V}\left(\mathrm{k}_{\mathrm{t}}, \eta_{\mathrm{t}}, \mathrm{w}, \mathrm{y}, \alpha\right)=\max \left[\mathrm{v}\left(\mathrm{k}_{\mathrm{t}}, \mathrm{k}_{\mathrm{t}+1}, \eta_{\mathrm{t}}, \mathrm{w}, \mathrm{y}, \alpha\right)+\gamma \sum_{\eta=1}^{\ell} \pi_{\eta} \mathrm{V}\left(\mathrm{k}_{\mathrm{t}+1}, \eta, \mathrm{w}, \mathrm{y}, \alpha\right)\right]
$$

subject to

$$
k_{t+1} \geq 0
$$

This approach can be generalized to allow events to be elements of a continuum. In this case $\mathrm{x}^{\mathrm{i}}(\mathrm{s})$ and $\mathrm{k}(\mathrm{s})$ are functions and we can rewrite both Euler equations and the dynamic programming functional equation using integrals. We can further generalize the probability distribution to be a Markov process. See Stokey, Lucas, and Prescott (1989) for details. 
Example 5.3 (5.2 Revisited). Consider an economy identical to that in Example 5.2 except that

$$
h\left(k_{t}, k_{t+1}, z_{1 t}, z_{2 t}, \eta_{t}\right)=\eta_{t} k_{t}^{\theta}\left(-z_{1 t}\right)^{1-\theta}-k_{t+1}-z_{2 t}
$$

where $\eta_{t}=\eta_{1}$ with probability $\pi_{1}$ and $\eta_{t}=\eta_{2}$ with probability $\pi_{2}$. The return function is

$$
v\left(k_{t}, k_{t+1}, \eta_{t}, w, y, \alpha\right)=\alpha y \log \left(\eta_{t} k_{t}^{\theta} w^{1-\theta}-k_{t+1}\right)-\alpha y \log y ;
$$

the Euler equation is

$$
-\frac{1}{\eta_{\mathrm{t}-1} \mathrm{k}_{\mathrm{t}-1}^{\theta} \mathrm{w}^{1-\theta}-\mathrm{k}_{\mathrm{t}}}+\gamma\left[\frac{\pi_{1} \eta_{1}^{\theta} \mathrm{k}_{\mathrm{t}}^{\theta-1} \mathrm{w}^{1-\theta}}{\eta_{1} \mathrm{k}_{\mathrm{t}}^{\theta} \mathrm{w}^{1-\theta}-\mathrm{k}_{\mathrm{t}+1}}+\frac{\pi_{2} \eta_{2}^{\theta} \mathrm{k}_{\mathrm{t}}^{\theta-1} \mathrm{w}^{1-\theta}}{\eta_{2} \mathrm{k}_{\mathrm{t}}^{\theta} \mathrm{w}^{1-\theta}-\mathrm{k}_{\mathrm{t}+1}}\right]=0 ;
$$

the value function is

$$
\begin{aligned}
\mathrm{V}\left(\mathrm{k}_{\mathrm{t}}, \eta_{\mathrm{t}}, \mathrm{w}, \mathrm{y}, \alpha\right)=\frac{\alpha \mathrm{y}}{1-\gamma}[\log (1-\gamma \theta) & +\frac{\gamma \theta}{1-\gamma \theta} \log \gamma \theta+\frac{\gamma}{1-\gamma \theta}\left(\pi_{1} \log \eta_{1}+\pi_{2} \log \eta_{2}\right) \\
& \left.+\frac{1-\gamma}{1-\gamma \theta} \log \eta_{\mathrm{t}}-\log \mathrm{y}+\frac{1-\theta}{1-\gamma \theta} \log \mathrm{w}+\frac{\theta-\gamma \theta}{1-\gamma \theta} \log \mathrm{k}_{\mathrm{t}}\right]
\end{aligned}
$$

and the policy function is

$$
g\left(k_{t}, \eta_{t}, w, y, \alpha\right)=\gamma \theta \eta_{\mathrm{t}} \mathrm{w}^{1-\theta_{\mathrm{k}}} \mathrm{k}_{\mathrm{t}}
$$

In the case where $\eta_{\mathrm{t}}$ is a continuous random variable, for example, when $\log \eta_{\mathrm{t}}$ is normally distributed with mean zero and variance $\sigma^{2}$, the Euler equation and the value function are easily modified and the policy function remains the same. (This latter feature does not hold in general, however.)

Once again our analysis can be extended in many directions. For an example of a stochastic equilibrium with recursive preferences that are not additively separable, see Epstein and Zin (1989).

\subsection{Differentiability and Regularity}

The analysis of the previous two sections allows us to easily extend the regularity analysis for static exchange economies to dynamic production economies. In economies where the value 
function $V\left(k_{1}, w, y, \alpha\right)$ is continuously twice differentiable $\left(C^{2}\right)$, the transfer function $t$ is continuously differentiable $\left(\mathrm{C}^{1}\right)$. In this case the analysis is very similar to that of static exchange economies: An equilibrium is a vector of welfare weights $\hat{\alpha}$ for which $t(\hat{\alpha})=0$, a system of $m$ equations in $m$ unknowns. Since $t$ is homogeneous of degree one, we can eliminate one unknown with a normalization. Since $\sum_{t=1}^{m} t_{i}(\alpha) \equiv 0$, we can eliminate one equation. A regular economy is one for which the Jacobian matrix of the transfer function $\operatorname{Dt}(\hat{\alpha})$ with the first row and column deleted, an $(m-1) \times(m-1)$ matrix, is nonsingular at every equilibrium. As with static exchange economy, a regular dynamic economy with production has a finite number of equilibria that vary continuously with the parameters of the economy. Kehoe, Levine, and Romer (1990) have extended Debreu's (1970) analysis to show that almost all economies with $C^{2}$ value functions are regular.

The problem has been to develop conditions that ensure that the value function is $C^{2}$. As we have mentioned, Benveniste and Scheinkman (1979) have shown that $V$ is $C^{1}$ under fairly general conditions on $u_{i}$ and $h$. Araujo and Scheinkman (1977) show that under suitable conditions on $u_{i}$ and $h$ that a $C^{2}$ value function follows from a global turnpike property. In other words, if all socially optimal paths converge to a unique steady state (which may depend on the welfare weights $\alpha$ ), then $\mathrm{V}$ is $\mathrm{C}^{2}$ and equilibria are generically determinate. Recently, Santos $(1989 \mathrm{a}, 1989 \mathrm{~b})$ has shown that $\mathrm{V}$ is $\mathrm{C}^{2}$ under far more general conditions. The most restrictive of these conditions serves only to ensure that $k_{t}$ remain uniformly separated from the boundary of $R_{+}^{n_{k}}$. (Montrucchio 1987, Boldrin and Montrucchio 1988, and Araujo 1989 present earlier partial results.) A different approach to proving that value functions are $C^{2}$ in stochastic economies has been pursued by Blume, Easley, and O'Hara (1982). 


\subsection{Computational Methods}

The ease of computing equilibria depends on how easy it is to solve dynamic programming problems. In this section we discuss two popular procedures, value function iteration and linear-quadratic approximation. These and a variety of other methods, together with applications to an example similar to Example 5.3, are discussed in a collection of papers edited by Taylor and Uhlig (1990).

Consider a dynamic programming problem expressed in terms of the functional equation

$$
\mathrm{V}\left(\mathrm{k}_{\mathrm{t}}, \mathrm{b}\right)=\max \left[\mathrm{v}\left(\mathrm{k}_{\mathrm{t}}, \mathrm{k}_{\mathrm{t}+1}, \mathrm{~b}\right)+\gamma \mathrm{V}\left(\mathrm{k}_{\mathrm{t}+1}, \mathrm{~b}\right)\right]
$$

subject to

$$
k_{t+1} \geq 0 \text {. }
$$

Here $b$ is a vector of parameters that includes the variables $(w, y, \alpha)$ discussed previously. The standard approach to proving the existence of a solution $V$ to this functional equation relies on the contraction mapping theorem.

Contraction Mapping Theorem. Let $S$ be a complete metric space with metric $d: S \rightarrow R_{+}$. Suppose that $\mathrm{T}: \mathrm{S} \rightarrow \mathrm{S}$ is a contraction with modulus $\beta$; that is, suppose that, for some $0<\beta<1$, $\mathrm{d}(\mathrm{Tx}, \mathrm{Ty}) \leq \beta \mathrm{d}(\mathrm{x}, \mathrm{y})$ for all $\mathrm{x}, \mathrm{y} \in \mathrm{S}$. Then there is a unique fixed point of $\mathrm{T}$ in $\mathrm{S}, \mathrm{x}=\mathrm{T} \&$.

The proof of this theorem suggests an algorithm for computing a fixed point: Start with $\mathrm{x}^{0} \in S$ and let $\mathrm{x}^{\mathrm{n}+1}=T \mathrm{x}^{\mathrm{n}}$. Since

$$
\mathrm{d}\left(\mathrm{x}^{\mathrm{n}+1}, \mathrm{x}^{\mathrm{n}+2}\right)=\mathrm{d}\left(\mathrm{Tx}^{\mathrm{n}}, \mathrm{Tx^{ \textrm {n } }}{ }^{\mathrm{1}}\right) \leq \beta \mathrm{d}\left(\mathrm{x}^{\mathrm{n}}, \mathrm{x}^{\mathrm{n}+1}\right)
$$

the sequence $x^{0}, x^{1}, \ldots$ is a Cauchy sequence and, since $S$ is complete, converges to a limit $x \in S$ that satisfies $d(\hat{x}, T \hat{x})=0$, or $\hat{x}=T \hat{x}$. To see that this fixed point is unique, suppose to the contrary, that there exists another fixed point $\hat{x}=\mathrm{T} \hat{\mathrm{R}}$. Then

$$
\mathrm{d}(\mathrm{TX}, \mathrm{T} \overline{\mathrm{x}})=\mathrm{d}(\mathrm{X}, \overline{\mathrm{x}})>\beta \mathrm{d}(\mathrm{X}, \overline{\mathrm{x}}),
$$


which contradicts T being a contraction. (See, for example, Luenberger 1969, Chapter 2, or Stokey, Lucas, and Prescott 1989, Chapter 3, for details.)

To apply this theorem to our dynamic programming problem, we need to give the space of functions $V(;, b)$ a topological structure. Let $S$ be the set of continuous bounded functions that map $\mathrm{R}_{+}^{\mathbf{n}_{\mathbf{k}}}$ into $\mathrm{R}$ with

$$
d\left(V^{1}, V^{2}\right)=\sup _{k}\left|V^{1}(k, b)-V^{2}(k, b)\right|
$$

$S$ is a complete metric space. There is a technical problem in that the return function $v$, and therefore the value function $V$, may not be bounded. We can get around this problem either by bounding $\mathrm{v}$ in a way that does not affect solutions or by using special properties of the mapping $\mathrm{T}$ (see, for example, Bertsekas 1976, Chapter 6, or Stokey, Lucas, and Prescott 1989, Chapter 4). In either case, the generalization is straightforward but technically tedious, and we shall ignore it.

Proposition 5.1 (Blackwell 1965). There exists a unique $V \in S$ that satisfies the functional equation of the dynamic programming problem.

Proof. Consider the mapping $\mathrm{T}: \mathrm{S} \rightarrow \mathrm{S}$ defined by

$$
(T V)\left(k_{t}, b\right)=\max \left[v\left(k_{t}, k_{t+1}, b\right)+\gamma V\left(k_{t+1}, b\right)\right]
$$

subject to

$$
k_{t+1} \geq 0
$$

If $\mathrm{v}$ and $\mathrm{V}$ are bounded and continuous, then so is TV. It therefore suffices to demonstrate that $\mathrm{T}$ is a contraction:

$$
\begin{aligned}
d\left(T V^{1}, T V^{2}\right) & =\sup _{k_{t}}\left|\max _{k_{t+1}}\left[v\left(k_{t}, k_{t+1}, b\right)+\gamma V^{1}\left(k_{t+1}, b\right)\right]-\max _{k_{t+1}}\left[v\left(k_{t}, k_{t+1}, b\right)+\gamma V^{2}\left(k_{t+1}, b\right)\right]\right| \\
& \leq \sup _{k_{t+1}}\left|\gamma V^{1}\left(k_{t+1}, b\right)-\gamma V^{2}\left(k_{t+1}, b\right)\right| .
\end{aligned}
$$


This last expression is just $\gamma d\left(V^{1}, V^{2}\right)$, which means that $T$ is a contraction with modulus $\gamma$.

Remark. It is easy to prove that $V$ satisfies such properties as being monotonically increasing and concave in $\mathrm{k}_{\mathrm{t}}$. Using the properties of $\mathrm{v}$, we can argue that, if $\mathrm{V}$ is monotonically increasing and concave, then so is TV; that is, $\mathrm{T}$ maps the subset of $\mathrm{S}$ whose elements are monotonically increasing and concave into itself. Since this set is itself a complete metric space, the fixed point $\hat{V}=T \hat{V}$ lies in it.

To implement this approach on the computer, we need to discretize the space of capital stocks. In particular, let $K=\left\{\mathrm{k}_{1}, \mathrm{k}_{2}, \ldots, \mathrm{k}_{\ell}\right\}, \mathrm{k}_{\mathrm{j}} \in \mathrm{R}_{+}^{\mathrm{n}_{\mathrm{k}}}$, be a finite, but large, set of capital stocks. The method of successive approximation treats $\mathrm{V}$ as an $\ell$ dimensional vector (see Bertsekas 1976 , Chapter 5). Starting with a simple guess for $V^{0}$, for example, $V^{0}\left(k_{j}, b\right)=0, j=1, \ldots, \ell$, we compute $\mathrm{V}^{\mathrm{n}+1}\left(\mathrm{k}_{\mathrm{j}}, \mathrm{b}\right)$ by solving

$$
\max v\left(k_{j}, k, b\right)+\gamma v^{n}(k, b)
$$

subject to

$$
\mathbf{k} \in \mathbf{K}
$$

The same argument as the proof of Proposition 5.1 establishes that this procedure is a contraction mapping. Furthermore, if the grid $\mathrm{K}$ is fine enough, then its fixed point is a good approximation to the true value function $V$. Variants on this method do not restrict $k$ to the finite set $K$ in the above maximization but interpolate or use polynomial approximations (see, for example, Tauchen 1990).

Unfortunately, although the method of successive approximation is guaranteed to converge, convergence can be very slow. An algorithm that greatly speeds convergence involves applying Newton's method to the equation

$$
\mathrm{V}=\mathrm{T}(\mathrm{V})
$$


where we think of $T$ as a mapping $R^{\ell}$ into $R^{\ell}$. This method is known as the policy iteration algorithm. (See Bertsekas 1976, Chapter 6; Rust 1987 and Christiano 1990 discuss variations and present economic applications.) The updating rule is

$$
\mathrm{V}^{\mathrm{n}+1}=\mathrm{V}^{\mathrm{n}}-\left[\mathrm{I}-\mathrm{DT}\left(\mathrm{V}^{\mathrm{n}}\right)\right]^{-1}\left[\mathrm{~V}^{\mathrm{n}}-\mathrm{T}\left(\mathrm{V}^{\mathrm{n}}\right)\right] .
$$

Although the $\ell \times \ell$ matrix $\left[I-D T\left(V^{n}\right)\right]$ is very large if the grid $\mathrm{K}$ is very fine, it typically contains a large number of zeros.

To apply these sorts of methods to stochastic problems, we must discretize the distribution of events $\eta$ if it is not already discrete. In the case of the method of successive approximation, for example, we compute $\mathrm{V}^{\mathrm{n}+1}\left(\mathrm{k}_{\mathrm{j}}, \eta_{\mathrm{j}}, \mathrm{b}\right)$ by solving

$$
\max v\left(k_{i}, k, \eta_{j}, b\right)+\gamma \sum_{\eta} \pi(\eta) \mathrm{V}^{\mathfrak{a}}(k, \eta, b)
$$

where $\mathrm{k}$ is restricted to the grid $\mathrm{K}$ and $\eta$ ranges over the discrete distribution of $\eta$ in the summation.

Example 5.4 (5.2 Revisited). Consider the functional equation

$$
\mathrm{V}\left(\mathrm{k}_{\mathrm{t}}, \mathrm{b}\right)=\max \left[\log \left(\mathrm{bk} \mathrm{k}_{\mathrm{t}}^{\theta}-\mathrm{k}_{\mathrm{t}+1}\right)+\gamma \mathrm{V}\left(\mathrm{k}_{\mathrm{t}+1}, \mathrm{~b}\right)\right]
$$

subject to

$$
\mathbf{k}_{\mathrm{t}+1} \geq 0
$$

Because of the special form of this problem we can solve for $V\left(k_{t}, b\right)$ analytically using the method of successive approximation. Let $V^{0}\left(k_{t}, b\right)=0$ for all $k_{t}, b$. Maximizing $\log \left(b k_{t}^{\theta}-k_{t+1}\right)$ with respect to $k_{t+1}$, we obtain the policy function

$$
\mathrm{g}^{1}\left(\mathrm{k}_{\mathrm{t}}, \mathrm{b}\right)=0
$$

and the value function

$$
v^{1}\left(k_{t}, b\right)=\log b+\theta \log k_{t} .
$$


Now maximizing $\left[\log \left(b k_{t}^{\theta}-k_{t+1}\right)+\gamma V^{1}\left(k_{t+1}, b\right)\right]$ we respect to $k_{t+1}$, we obtain the policy function

$$
\mathbf{g}^{2}\left(\mathrm{k}_{\mathrm{t}}, \mathrm{b}\right)=[\gamma \theta \mathrm{b} /(1+\gamma \theta)] \mathrm{k}_{\mathrm{t}}^{\theta}
$$

and the value function

$$
\mathrm{V}^{2}\left(\mathrm{k}_{\mathrm{t}}, \mathrm{b}\right)=\log \frac{\mathrm{b}}{1+\gamma^{\theta}}+\gamma \log \mathrm{b}+\gamma \theta \log \frac{\gamma \theta \mathrm{b}}{1+\gamma \theta}+\theta(1+\gamma \theta) \log \mathrm{k}_{\mathrm{t}^{+}}
$$

In general, at iteration $\mathrm{n}$ we obtain the policy function

$$
\mathbf{g}^{\mathbf{n}}\left(k_{t}, b\right)=\left[\left(1-(\gamma \theta)^{\mathbf{n}-1}\right) /\left(1-(\gamma \theta)^{\mathbf{n}}\right)\right] \gamma \theta b k_{t}^{\theta}
$$

and the value function

$$
\begin{aligned}
\mathrm{V}^{\mathrm{n}}\left(\mathrm{k}_{\mathbf{t}}, \mathrm{b}\right)= & \sum_{\mathbf{i}=1}^{\mathrm{n}} \gamma^{\mathrm{i}-1} \log \left[\mathrm{b}(1-\gamma \theta) /\left(1-(\gamma \theta)^{\mathrm{n}-\mathrm{i}+1}\right)\right] \\
& +\gamma \theta \sum_{i=1}^{\mathrm{n}-1} \gamma^{i-1}\left[\left(1-(\gamma \theta)^{\mathrm{n}-\mathbf{i}}\right) /(1-\gamma \theta)\right] \log \left[\gamma \theta b\left(1-(\gamma \theta)^{\mathrm{n}-\mathrm{i}}\right) /\left(1-(\gamma \theta)^{\mathbf{n}-\mathbf{i}+1}\right)\right] \\
& +\theta\left[\left(1-(\gamma \theta)^{\mathrm{n}}\right) /(1-\gamma \theta)\right] \log \mathrm{k}_{\mathbf{t}} .
\end{aligned}
$$

In the limit these converge to

$$
\begin{aligned}
& \mathrm{g}\left(\mathrm{k}_{\mathrm{t}}, \mathrm{b}\right)=\gamma \theta \mathrm{b} \mathrm{k}_{\mathrm{t}}^{\theta} \\
& \mathrm{V}\left(\mathrm{k}_{\mathrm{t}}, \mathrm{b}\right)=\frac{1}{1-\gamma}\left[\log \mathrm{b}(1-\gamma \theta)+\frac{\gamma \theta}{1-\gamma^{\theta}} \log \gamma^{\theta b}\right]+\frac{\theta}{1-\gamma^{\theta}} \log \mathrm{k}_{\mathrm{t}^{*}}
\end{aligned}
$$

Writing

$$
\begin{aligned}
& \mathbf{g}^{\mathrm{n}}\left(\mathrm{k}_{\mathrm{t}}, \mathrm{b}\right)=\mathrm{a}_{0}^{\mathrm{n}_{\mathrm{t}}^{\theta}} \mathrm{k}_{t} \\
& \mathrm{~V}^{\mathrm{n}}\left(\mathrm{k}_{\mathrm{t}}, \mathrm{b}\right)=\mathrm{a}_{1}^{\mathrm{n}}+\mathrm{a}_{2}^{\mathrm{n}} \log \mathrm{k}_{\mathrm{t}},
\end{aligned}
$$

we compute successive approximations for the problem with $\gamma=0.95, \theta=0.3$, and $\mathrm{b}=2$ : 


\begin{tabular}{|llrl|}
\hline $\mathrm{n}$ & \multicolumn{1}{c}{$\mathrm{a}_{0}^{\mathrm{n}}$} & \multicolumn{1}{c|}{$\mathrm{a}_{1}^{\mathrm{n}}$} & \multicolumn{1}{c|}{$\mathrm{a}_{2}^{\mathrm{n}}$} \\
\hline 1 & 0 & 0.693147 & 0.3 \\
2 & 0.443580 & 0.869208 & 0.3855 \\
5 & 0.567306 & 1.150158 & 0.418791 \\
10 & 0.569995 & 1.719038 & 0.419579 \\
$\infty$ & 0.57 & 2.672261 & 0.419580 \\
\hline
\end{tabular}

Remark. Notice that the successive approximations for $\mathrm{V}$ converge monotonically. It is a general property of this algorithm that, if $V^{1}(k, b)>V^{0}(k, b)$ for all $k$, then $V^{n+1}(k, b)>V^{n}(k, b)$ for all k. This property, which is the result of $v\left(k_{t}, k_{t+1}, b\right)$ being increasing in $k_{t}$, is frequently useful for proving convergence when $\mathrm{v}$ is unbounded and for developing more efficient algorithms (see, for example, Bertsekas 1976, Chapter 6).

Value function iteration methods can be very costly in terms of computer time when the number of state variables, $\mathrm{n}_{\mathrm{k}}$, is more than two or three or when there is uncertainty. Another solution method that is popular in applications is to solve the linear-quadratic approximation to the original problem. (See, for example, Bertsekas 1976, Chapter 4; Kydland and Prescott 1980, 1982; and Sargent 1987.) The idea is to approximate the return function $v\left(k_{t}, k_{t+1}, b\right)$ by a quadratic function or, equivalently, to linearize the Euler equations. The approximate model can then be solved exactly.

We start by computing a steady state, a capital stock $\bar{k} \in \mathbf{R}_{+}^{\mathbf{n}_{\mathbf{k}}}$ that satisfies the Euler equation

$$
\mathrm{D}_{2} \mathrm{v}(\overline{\mathrm{k}}, \overline{\mathrm{k}}, \mathrm{b})+\gamma \mathrm{D}_{1} \mathrm{v}(\overline{\mathrm{k}}, \overline{\mathrm{k}}, \mathrm{b})=0 .
$$

The problem of computing a steady state can be easily formulated as a fixed point problem. Let $\mathrm{S}=$ $\left\{k \in \mathrm{R}_{+}^{\mathbf{n}_{\mathbf{k}}} \mid\|\mathrm{k}\| \leq\left\|\mathrm{k}^{\mathrm{max}}\right\|\right\}$ and let $\kappa(k)$ be the vector $\kappa \in \mathrm{R}^{\mathbf{n}_{\mathbf{k}}}$ that solves 


$$
\max v(\mathbf{k}, \kappa, \mathrm{b})+\gamma v(\kappa, \mathrm{k}, \mathrm{b})
$$

subject to

$$
\begin{aligned}
& \|\kappa\| \leq\left\|\mathrm{k}^{\max }\right\| \\
& \kappa \geq 0 .
\end{aligned}
$$

Notice that $k(\mathrm{k})$ is continuous and maps $S$ into $S$ and that $S$ is nonempty, compact, and convex. Consequently, $\kappa$ has a fixed point $\overline{\mathrm{k}}=\kappa(\overline{\mathrm{k}})$ that can be computed using the methods of Section 2 . Unfortunately, although the dynamic programming problem itself always has a unique solution because of the concavity of $v$, there may be multiple steady states (see Burmeister 1980 , Chapter 4 , for a discussion).

We approximate $v\left(k_{t}, k_{t+1}, b\right)$ by the second-order Taylor series

$$
\begin{aligned}
\tilde{v}\left(x_{t}, x_{t+1}\right)=v & +D_{1} v x_{t}+D_{2} v x_{t+1} \\
& +(1 / 2)\left(x_{t}^{\prime} D_{11} v x_{t}+x_{t+1}^{\prime} D_{22} v x_{t+1}+x_{t}^{\prime} D_{12} v x_{t+1}+x_{t+1}^{\prime} D_{21} v x_{t}\right)
\end{aligned}
$$

Here, for example, $D_{1} v$ is the $1 \times n_{k}$ gradient vector of $v$ with respect to its first vector of arguments evaluated at $(\overline{\mathrm{k}}, \overline{\mathrm{k}}, \mathrm{b})$ and $D_{11} v$ is the $n_{k} \times n_{k}$ matrix of second derivatives; also $x_{t}=k_{t}-\bar{k}$. There are two approaches to solving the dynamic programming problem with $v$ replaced by its quadratic approximation $\vec{v}$. The first is like the method in Example 5.2: we guess the functional form of $\tilde{V}\left(x_{t}\right)$, in this case a quadratic,

$$
\tilde{V}\left(x_{t}\right)=a_{1}+a_{2}^{\prime} x_{t}+(1 / 2) x_{t}^{\prime} A_{3} x_{t}
$$

where $a_{1}$ is $1 \times 1, a_{2}$ is $n_{k} \times 1$, and $A_{3}$ is $n_{k} \times n_{k}$, and then solve for $a_{1}, a_{2}$, and $A_{3}$. The second method is to directly attack the linear Euler equations

$$
D_{21} v x_{t-1}+\left(\gamma D_{11} v+D_{22} v\right) x_{t}+\gamma D_{12} v x_{t+1}=0
$$

If we follow the first method, we solve

$$
\max \tilde{v}\left(x_{t}, x_{t+1}\right)+\gamma\left(a_{1}+a_{2}^{\prime} x_{t}+(1 / 2) x_{t}^{\prime} A_{3} x_{t}\right)
$$

to find 


$$
x_{t+1}=-\left(D_{22} v+\gamma A_{3}\right)^{-1}\left(D_{21} v x_{t}+\gamma a_{2}+D_{2} v^{\prime}\right)
$$

Since we know that $x_{t}=0$ implies $x_{t+1}=0$ because $\vec{k}$ is a steady state, we can easily solve

$$
\begin{aligned}
& a_{2}=-\gamma^{-1} D_{2} v^{\prime}=D_{1} v^{\prime} \\
& a_{1}=v /(1-\gamma) .
\end{aligned}
$$

Plugging $x_{t+1}=-\left(D_{22} v+\gamma A_{3}\right)^{-1} D_{21} v x_{t}$ into the functional equation that defines $\tilde{v}$, we obtain the matrix equation

$$
A_{3}=D_{11} v-D_{12} v\left(D_{22} v+\gamma A_{3}\right)^{-1} D_{21} v
$$

often called the Riccati equation. One approach to solving this is to guess $A_{3}^{0}$ and iterate

$$
A_{3}^{n}=D_{11} v-D_{12} v\left(D_{22} v+\gamma A_{3}^{n-1}\right)^{-1} D_{21} v .
$$

Bertsekas (1976, Chapter 3) presents conditions under which this algorithm converges.

Alternatively, we could turn the linear Euler equations into the system of $2 n_{\mathrm{k}}$ first order difference equations

$$
\left[\begin{array}{c}
x_{t} \\
x_{t+1}
\end{array}\right]=\left[\begin{array}{cc}
0 & I \\
-\gamma^{-1} D_{12} v^{-1} D_{21} v & -D_{12} v^{-1}\left(D_{11}+\gamma^{-1} D_{22} v\right)
\end{array}\right]\left[\begin{array}{c}
x_{t-1} \\
x_{t}
\end{array}\right] .
$$

Denote the $2 n_{k} \times 2 n_{k}$ on the right side of the equation as $G$. As long as the eigenvalues of $G$ are distinct, we can write $G=X \Lambda X^{-1}$, where $\Lambda$ is the $2 n_{k} \times 2 n_{k}$ diagonal matrix of eigenvalues of $G$ and $X$ is the $2 n_{k} \times 2 n_{k}$ matrix whose columns are the associated eigenvectors. The theory for systems with repeated eigenvalues is similar but more tedious; we ignore this possibility, however, because it is easy to show that having distinct eigenvalues holds for almost all $v$ (see, for example, Hirsch and Smale 1974, Chapter 7). Writing 


$$
\left[\begin{array}{c}
x_{t} \\
x_{t+1}
\end{array}\right]=G^{t-1}\left[\begin{array}{l}
x_{1} \\
x_{2}
\end{array}\right]=X^{t-1} X^{-1}\left[\begin{array}{l}
x_{1} \\
x_{2}
\end{array}\right] \text {, }
$$

we see that $x_{t}$ converges to zero if and only if the $2 n_{k} \times 1$ vector

$$
c=x^{-1}\left[\begin{array}{l}
x_{1} \\
x_{2}
\end{array}\right]
$$

has nonzero elements only where the corresponding eigenvalues $\lambda_{i}$ has modulus less than one. This means that the vector $\left(x_{1}, x_{2}\right)$ must lie in the subspace of $R^{n_{k}}$, called the stable subspace of $G$, spanned by the (possibly) complex eigenvectors of $G$ associated with eigenvalues less than one in modulus.

An easy way to see this is to rewrite the above relationship as

$$
\left[\begin{array}{c}
x_{t} \\
x_{t+1}
\end{array}\right]=\sum_{i=1}^{2 n_{k}} c_{i} \lambda_{i}^{t-1}\left[\begin{array}{c}
z_{i} \\
\lambda_{i} z_{i}
\end{array}\right]
$$

where $\left(z_{i}, \lambda_{i} z_{i}\right) \in C^{2 n}$ is the eigenvector associated with $\lambda_{i}$ and also write

$$
\left[\begin{array}{l}
x_{1} \\
x_{2}
\end{array}\right]=\sum_{i=1}^{2 n_{i}} c_{i}\left[\begin{array}{c}
z_{i} \\
\lambda_{i} z_{i}
\end{array}\right]
$$

For $x_{t}$ to converge to zero, the vector $c$ must be such that $\left(x_{1}, x_{2}\right)$ is a linear combination of eigenvectors associated with stable eigenvalues. If $x_{t}$ converges to zero, then $k_{t}=x_{t}+\vec{k}$ satisfies the transversality conditions

$$
\lim _{t \rightarrow \infty} \gamma^{t-1} D_{1} v(\bar{k}, \bar{k}, b) k_{t}=0
$$

A case of particular interest is when exactly half, $n_{k}$, of the eigenvalues of $G$ are stable and the other half are unstable. This case is often called saddlepoint splitting of the eigenvalues. If this condition holds, we can uniquely solve 


$$
\mathrm{x}_{1}=\sum_{\mathrm{i}=1}^{2 \mathrm{n}_{\mathrm{k}}} \mathrm{c}_{\mathrm{i}} \mathrm{z}_{\mathrm{i}}
$$

for the $n_{k}$ constants $c_{i}$ associated with the stable eigenvectors and use the result to uniquely determine $x_{2}$. There would be a continuum of solutions if there were more than $n_{k}$ stable eigenvalues. If, however, there were fewer than $n_{k}$ stable eigenvalues, then there would be, in general, no solution at all that converges to the steady state.

Since $v$ and its quadratic approximation $\tilde{\mathrm{v}}$ are concave, we would not expect there to be multiple solutions to the dynamic programming problem. (Remember, however, that there may be multiple equilibria corresponding to different welfare weights $\alpha$.) To see that there cannot be more than $n_{k}$ stable eigenvalues of $G$, we can write the characteristic equation as

$$
\operatorname{det}\left[D_{21} v+\lambda\left(\gamma D_{11} v+D_{22} v\right)+\lambda^{2} \gamma D_{12} v\right]=0 .
$$

Suppose that there is a vector $\mathrm{x} \in \mathrm{R}^{\mathbf{n}_{\mathbf{k}}}$ such that

$$
\left[D_{21} v+\lambda\left(\gamma D_{11} v+D_{22} v\right)+\lambda^{2} \gamma D_{12} v\right] x=0
$$

Then, since $D_{11} v$ and $D_{22} v$ are symmetric and $D_{21} v=D_{12} v^{\prime}$,

$$
\mathrm{x}^{\prime}\left[\mathrm{D}_{21} \mathrm{v}+\mu\left(\gamma \mathrm{D}_{11} \mathrm{v}+\mathrm{D}_{22} \mathrm{v}\right)+\mu \gamma \mathrm{D}_{12} \mathrm{v}\right]=0
$$

where $\mu=(\gamma \lambda)^{-1}$. Consequently, if $\lambda$ is an eigenvalue of $G$, so is $(\gamma \lambda)^{-1}$. This implies that $n_{k}$ eigenvalues are smaller than $\gamma^{-1 / 2}>1$ in modulus and $n_{k}$ eigenvalues are larger.

If $\gamma$ is close to one, then we would expect the eigenvalues of $G$ to exhibit saddlepoint splitting. Indeed, there are turnpike theorems that guarantee convergence to a steady state in this case (see McKenzie 1987 for a survey of such results). If $\gamma$ is not close to one, there may be less than $n_{k}$ stable eigenvalues, and the solution may converge to a cycle or even exhibit chaotic dynamics (see, for example, Ryder and Heal 1973, Benhabib and Nishumura 1979, and Boldrin and Montrucchio 1986). Indeed, Boldrin and Montrucchio (1986) prove that, if $K$ is a compact subset 
of $\mathrm{R}^{\mathrm{n}_{\mathrm{k}}}$ and $\mathrm{g}: \mathrm{K} \rightarrow \mathrm{K}$ is twice continuously differentiable, then there exists a return function $\mathrm{v}$ and a discount factor $\gamma$ such that $\mathrm{g}$ is the optimal policy function. Since the dynamics of the solution path are governed solely by the policy function, this says that equilibrium dynamics are arbitrary. The construction used in the proof of this theorem, not surprisingly, relies on small discount factors.

Example 5.5 (5.2 Revisited). The return function $v\left(k_{t}, k_{t+1}, b\right)=\log \left(b k_{t}^{\theta}-k_{t+1}\right)$ can be linearized around the steady state $\overline{\mathrm{k}}=(\gamma \theta \mathrm{b})^{1 /(1-\theta)}$ as

$$
\begin{aligned}
\tilde{\mathrm{v}}\left(\mathrm{x}_{\mathrm{t}}, \mathrm{x}_{\mathrm{t}+1}\right)=\log \overline{\mathrm{c}} & +(\gamma \overline{\mathrm{c}})^{-1} \mathrm{x}_{\mathrm{t}}-\overline{\mathrm{c}}^{-1} \mathrm{x}_{\mathrm{t}+1} \\
& -(1 / 2)\left[\left((1-\theta)(\gamma \overline{\mathrm{c}} \overline{\mathrm{k}})^{-1}+(\gamma \overline{\mathrm{c}})^{-2}\right) \mathrm{x}_{\mathrm{t}}^{2}+\overline{\mathrm{c}}^{-2} \mathrm{x}_{\mathrm{t}+1}^{2}-2 \gamma^{-1} \overline{\mathrm{c}}^{-2} \mathrm{x}_{\mathrm{t}} \mathrm{x}_{\mathrm{t}+1}\right]
\end{aligned}
$$

Here $\bar{c}=b \bar{k}^{\theta}-\bar{k}$. The value function $\tilde{v}\left(k_{t}\right)=a_{1}+a_{2} x_{t}+(1 / 2) a_{3} x_{t}^{2}$ is such that $a_{1}=$ $(\log \bar{c}) /(1-\theta), a_{2}=(\gamma \bar{c})^{-1}$, and $a_{3}$ can be solved for using the Riccati equation

$$
\mathrm{a}_{3}=-\left((1-\theta)(\gamma \overline{\mathrm{c}} \overline{\mathrm{k}})^{-1}+(\gamma \overline{\mathrm{c}})^{-2}\right)-\gamma^{-2} \overline{\mathrm{c}}^{-4}\left(\gamma \mathrm{a}_{3}-\overline{\mathrm{c}}^{-2}\right)^{-1} \text {. }
$$

There are two solutions to this equation, $a_{3}=-(1-\gamma \theta) \gamma^{-2} \theta^{-1} \bar{c}^{-2}$ and $a_{3}=(1-\theta) \gamma^{-1} \bar{c}^{-2}$, the first of which is the nonpositive number (in general, negative semidefinite matrix) that we need for $\tilde{\mathrm{V}}$ to be concave. The policy function is $\tilde{\mathrm{g}}\left(\mathrm{x}_{\mathrm{t}}\right)=\theta \mathrm{x}_{\mathrm{t}}$.

An alternative for finding this policy function is to analyze the Euler equation

$$
\overline{\mathrm{c}}^{-2} \mathrm{x}_{\mathrm{t}+1}-\left((1-\theta)(\overline{\mathrm{c}} \overline{\mathrm{k}})^{-1}+\gamma^{-1} \overline{\mathrm{c}}^{-2}+\overline{\mathrm{c}}^{-2}\right) \mathrm{x}_{\mathrm{t}}+\gamma^{-1} \overline{\mathrm{c}}^{-2} \mathrm{x}_{\mathrm{t}-1}=0
$$

The corresponding characteristic equation has two roots, $\lambda=\theta$ and $\lambda=(\gamma \theta)^{-1}$, only the first of which satisfies $|\lambda|<1$.

To see the relative accuracy of the linear-quadratic approximation, we again consider the model where $\gamma=0.95, \theta=0.3$, and $b=2$ and solve for the equilibrium path for $\mathrm{k}_{\mathrm{t}}$ starting at $\mathrm{k}_{1}=0.1$ using both the linear-quadratic policy function, $\overrightarrow{\mathrm{k}}+\theta\left(\mathrm{k}_{\mathrm{t}}-\overline{\mathrm{k}}\right)$, and the true policy function, $\gamma \theta \mathrm{bk} \mathrm{k}_{\mathrm{t}}^{\theta}$ 


\begin{tabular}{||lll|}
\hline & Exact & Linear-Quadratic \\
\hline 1 & 0.1 & 0.1 \\
2 & 0.285677 & 0.343580 \\
3 & 0.391415 & 0.416654 \\
4 & 0.430196 & 0.438576 \\
5 & 0.442563 & 0.445152 \\
10 & 0.447958 & 0.447964 \\
$\infty$ & 0.447971 & 0.447971 \\
\hline
\end{tabular}

Remark. This example has the property that the $\tilde{\mathrm{V}}$ is the second-order Taylor series approximation to $\mathrm{V}$ and $\mathrm{g}$ is the first-order approximation to $\mathrm{g}$; this is not a general feature of this method. An alternative approach is to use the linear-quadratic approximation where $x_{t}=\left(\log k_{t}-\log \bar{k}\right)$. For our specific example, such an approximation is exact as can be easily verified.

Another promising approach to approximating solutions to dynamic programming problems relies on restricting the policy functions that solve the Euler equations to a finite dimensional function space. The linear-quadratic approximation restricts the policy function to being linear. Other possibilities include polynomials of a fixed finite order or various finite sequences of polynomial and trigonometric functions. For an exposition and economic applications of these methods, known as minimum weighted residual methods, see Judd (1991). A closely related method, which parameterizes the expectations of next period's value function, rather than the policy function, using a finite dimensional approximation to the function space, has been proposed by den Haan and Marcet (1990).

\section{Overlapping Generations Economies}

Even though they have an infinite number of goods, the economies in the previous section are relatively easy to study because the two welfare theorems allow us to characterize their equilibria 
as solutions to welfare maximization problems. Many dynamic economies have equilibria that do not solve such maximization problems. An important example is the overlapping generations model originally studied by Samuelson (1958). Frequently, to approximate equilibria of such an economy on the computer, we must truncate it so that it has a finite number of goods. Studying the properties of economies with infinite numbers of goods, however, provides insights into the properties of those with large, but finite, numbers of goods.

\subsection{Existence of Equilibrium}

To keep the discussion simple we focus on exchange economies. Overlapping generations economies with production can be analyzed using a similar approach (see, for example, Burke 1986 and Muller and Woodford 1988). Consider a stationary economy with $\mathrm{n}$ goods in each period and $\mathrm{m}$ consumers who live for two periods in the generation born in period $t, t=1,2, \ldots$ In addition there are $m_{0}$ consumers, generation 0 , who live only in the first period. Balasko, Cass, and Shell (1980) present a simple procedure for converting a model in which consumers live for $k$ periods into one in which they live for two: Redefine generations $-k+2,-k+1, \ldots, 0$ to be generation 0 , generations $1,2, \ldots, k-1$ to be generation 1 , and so on. Similarly, redefine periods. Notice that the number of goods in each period and the number of consumers in each generation increase by a factor of $k-1$. The important feature of the procedure is that each redefined generation lives for two redefined periods.

Each consumer in generation thas a utility function $\mathrm{u}_{\mathrm{i}}: \mathrm{R}_{+}^{2 \mathrm{n}} \rightarrow \mathrm{R}$ that is strictly concave and monotonically increasing and an endowment $\left(w_{1}^{i}, w_{2}^{i}\right) \in R_{++}^{2 n}$. Faced with prices $p_{t}, p_{t+1}$, the consumer solves

$$
\max u_{i}\left(x_{t}, x_{t+1}\right)
$$

stubject to 


$$
\begin{aligned}
& p_{t}^{\prime} x_{t}+p_{t+1}^{\prime} x_{t+1} \leq p_{t}^{\prime} w_{1}^{i}+p_{t+1}^{\prime} w_{2}^{i} \\
& x_{t}, x_{t+1} \geq 0 .
\end{aligned}
$$

Each consumer in generation 0 solves the problem

$$
\max u_{\mathrm{i} 0}\left(\mathrm{x}_{1}\right)
$$

subject to

$$
\begin{aligned}
& \mathrm{p}_{1}^{\prime x_{1}} \leq \mathrm{p}_{1}^{\prime w_{2}^{i 0}}+\mathrm{M}_{\mathrm{i}} \\
& \mathrm{x}_{1} \geq 0 .
\end{aligned}
$$

Here $\mathrm{M}_{\mathrm{i}}$ is the consumer's endowment of fiat money, which can be positive, negative, or zero.

We define the aggregate excess demand functions

$$
\begin{aligned}
& z_{0}\left(p_{1}\right)=\sum_{i=1}^{m_{0}}\left(x_{1}^{i 0}\left(p_{1}\right)-w_{2}^{i 0}\right) \\
& y\left(p_{t}, p_{t+1}\right)=\sum_{i=1}^{m}\left(x_{t}^{i t}\left(p_{t}, p_{t+1}\right)-w_{1}^{i}\right) \\
& z\left(p_{t}, p_{t+1}\right)=\sum_{i=1}^{m}\left(x_{t+1}^{i t}\left(p_{t}, p_{t+1}\right)-w_{2}^{i}\right) .
\end{aligned}
$$

These functions are continuous at least for strictly positive prices; they are bounded below by $-\Sigma_{i=1}^{m_{0}} w_{2}^{i 0},-\Sigma_{i=1}^{m} w_{1}^{i}$, and $-\sum_{i=1}^{m} w_{2}^{i}$, respectively; $y$ and $z$ are homogeneous of degree zero while $z_{0}$ is homogeneous of degree zero if and only if $M_{i}=0, i=1, \ldots, m_{0}$; and $z_{0}, y$, and $z$ obey the following versions of Walras's law:

$$
\begin{aligned}
& p_{1}^{\prime} z_{0}\left(p_{1}\right)=\sum_{i=1}^{m_{0}} M_{i} \\
& p_{t}^{\prime} y\left(p_{t}, p_{t+1}\right)+p_{t+1}^{\prime} x\left(p_{t}, p_{t+1}\right) \equiv 0
\end{aligned}
$$


An equilibrium of this economy is a price sequence $\hat{p}_{1}, \hat{f}_{2}, \ldots$ such that

$$
\begin{aligned}
& z_{0}\left(\hat{p}_{1}\right)+y\left(\hat{p}_{1}, \hat{p}_{2}\right) \leq 0 \\
& z\left(\hat{p}_{t-1}, \hat{p}_{t}\right)+y\left(\hat{p}_{t}, \hat{P}_{t-1}\right) \leq 0, \quad t=2,3, \ldots
\end{aligned}
$$

and that there is equality whenever the price $p_{i t}$ is strictly positive.

Consider a truncated version of this economy, an economy that ends in period $\mathrm{T}$. There is a terminal young generation with excess demand function $y_{T}\left(p_{T}\right)$ that satisfies

$$
\mathrm{p}_{\mathrm{T}}^{\prime} \mathrm{y}_{\mathrm{T}}\left(\mathrm{p}_{\mathrm{T}}\right) \equiv-\sum_{\mathrm{i}=1}^{\mathrm{m}_{0}} \mathrm{M}_{\mathrm{i}}
$$

There are many ways of constructing such a function. One is to set

$$
\mathrm{y}_{\mathrm{T}}\left(\mathrm{p}_{\mathrm{T}}\right)=-\left[\sum_{\mathrm{i}=1}^{\mathrm{m}_{0}} \mathrm{M}_{\mathrm{i}} /\left(\mathrm{e}^{\prime} \mathrm{p}_{\mathrm{T}}\right)\right] \mathrm{e} .
$$

This is now a finite economy with transfer payments.

To prove the existence of equilibria we consider a sequence of truncated economies where the truncation date $\mathrm{T}$ tends to infinity. To keep our discussion simple we focus on economies without fiat money. In the simple economy that we study here, this is often the only case in which we can prove existence (see, for example, Balasko, Cass, and Shell 1980). There are, however, modified versions of this economy in which the only equilibria that exist involve nonzero quantities of fiat money (see Wilson 1981 and Burke 1988).

In a truncated economy without fiat money existence of equilibrium follows directly from the arguments in Section 2.1. To ensure that the equilibrium does not involve prices being all equal to zero in any period, we employ two additional assumptions: first, there exists $\alpha>0$ and $\mathrm{B}>$ $\max \left[\sum_{i=1}^{m_{0}} w_{0}^{i}+\sum_{i=1}^{m} w_{1}^{i}, \sum_{i=1}^{m}\left(w_{1}^{i}+w_{2}^{i}\right)\right]$ such that $e^{\prime} p_{t+1} / e^{\prime} p_{t}<\alpha$ implies $\left\|z\left(p_{t}, p_{t+1}\right)\right\|>B ;$ 
second, there exists $\bar{\alpha}>\underline{\alpha}$ such that $\mathrm{e}^{\prime} \mathrm{p}_{\mathrm{t}+1} / \mathrm{e}^{\prime} \mathrm{p}_{\mathrm{t}}>\bar{\alpha}$ implies $\left\|\mathrm{y}\left(\mathrm{p}_{\mathrm{t}}, \mathrm{p}_{\mathrm{t}+1}\right)\right\|>\mathrm{B}$. See Balasko, Cass, and Shell (1980) for conditions on preferences and endowments that imply these conditions.

Proposition 6.1 (Balasko, Cass, and Shell 1980 and Wilson 1981). There exists an equilibrium $\hat{p}_{1}$, $\hat{p}_{2}, \ldots$ for the overlapping generations model without fiat money.

Proof. Consider the economy truncated at period T. Proposition 2.1 and Brouwer's fixed point theorem imply that it has an equilibrium $\left(\hat{\rho}_{1}, \ldots, \hat{\mathrm{P}}_{\mathrm{T}}\right)$. Consider now the sequence of prices $\mathrm{p}_{\mathrm{t}}^{\mathrm{T}}=$ $\hat{\mathrm{p}}_{\mathrm{t}} /\left(\mathrm{e}^{\prime} \hat{\mathrm{p}}_{1}\right), \mathrm{t}=1, \ldots, \mathrm{T}, \mathrm{p}_{\mathrm{t}}^{\mathrm{T}}=\mathrm{e}, \mathrm{t}=\mathrm{T}+1, \mathrm{~T}+2, \ldots$ Our assumptions on $\mathrm{y}$ and $\mathrm{z}$ imply that $\mathrm{p}_{\mathrm{t}}^{\mathrm{T}}, \mathrm{t}=1,2, \ldots$, is an element of the nonempty, compact, and convex set

$$
S_{t}=\left\{p \in R^{n} \mid p \geq 0, \alpha^{t-1} \leq e^{\prime} p \leq \bar{\alpha}^{t-1}\right\}
$$

The product $\mathrm{II}_{\mathrm{t}=1}^{\infty} S_{\mathrm{t}}$ is compact in the product topology. Consequently, the sequence (of sequences) $\mathrm{p}_{1}^{1}, \mathrm{p}_{2}^{1}, \ldots, \mathrm{p}_{1}^{2}, \mathrm{p}_{2}^{2}, \ldots$ has a convergent subsequence. Let $\overline{\mathrm{p}}_{1}, \overline{\mathrm{p}}_{2}, \ldots$ be the limit of this subsequence. Notice that, since the functions $\mathrm{z}_{0}, \mathrm{y}$, and $\mathrm{z}$ involve only a finite number of variables, the functions $z_{0}+y$ and $z+y, t=2,3, \ldots$, are continuous in the product topology. Consequently, since each sequence $p_{1}^{T}, p_{2}^{T}, \ldots$ satisfies the equilibrium conditions in periods $t=1,2, \ldots$, $T-1$, the sequence $\bar{p}_{1}, \bar{p}_{2}, \ldots$ satisfies them in all periods.

Remark. The only role played by stationarity, the assumption that $y$ and $z$ do not change over time, is to provide the bounds $\alpha$ and $\vec{\alpha}$. Consequently, this result applies to nonstationary economies in which such bounds are assumed to hold. In particular, it applies to economies that are stationary after a given date. 


\subsection{Multiplicity of Equilibria}

Unfortunately, in contrast to economies with a finite number of infinitely lived consumers, there are robust examples of overlapping generations economies with an infinite number of equilibria. As we shall see, this has strong implications for computational methods for approximating equilibria, including methods that truncate the economy at a finite date. Our discussion of multiplicity follows Gale (1973) and Kehoe and Levine (1985, 1990b).

Ignoring the possibility of free goods, we can write the equilibrium conditions as

$$
\begin{aligned}
& z_{0}\left(p_{1}\right)+y\left(p_{1}, p_{2}\right)=0 \\
& z\left(p_{t-1}, p_{t}\right)+y\left(p_{t}, p_{t+1}\right)=0, \quad t=2,3, \ldots
\end{aligned}
$$

Once $\mathrm{p}_{1}$ and $\mathrm{p}_{2}$ are determined, the second condition acts as a nonlinear difference equation determining $p_{t+1}$ as a function of $\left(p_{t-1}, p_{t}\right)$. In this section we focus attention on the behavior of

equilibrium price paths near a steady state $p_{t}=\beta^{t-1} p$, where $p \in R_{+}^{n} \backslash\{0\}$ and $\beta \in R_{++}$, to the second condition,

$$
z\left(\beta^{t-2} p, \beta^{t-1} p\right)+y\left(\beta^{t-1} p, \beta^{t} p\right)=z(p, \beta p)+y(p, \beta p)=0 .
$$

We are interested in prices that converge to a steady state, $\left(p_{t}, p_{t+1}\right) /\left\|\left(p_{t}, p_{t+1}\right)\right\| \rightarrow(p, \beta p) /\|(p, \beta p)\|$ as $t \rightarrow \infty$.

Focusing on prices that start and remain in some neighborhood of a steady state has two advantages: First, the implicit function theorem says that, if $D_{2} y(p, \beta p)$ is nonsingular, a condition that Kehoe and Levine (1984) holds almost always, $\mathrm{p}_{t+1}$ can be calculated as a unique, continuously differentiable function of $\left(p_{t-1}, p_{t}\right)$ in some neighborhood of $(p, \beta p)$. There may be multiple solutions $\mathrm{p}_{\mathbf{t}+1}$ globally, however. Second, the local stable manifold theorem says that the qualitative behavior of the nonlinear dynamical system generated by the equilibrium conditions is almost always the same as that of its linear approximation (see Scheinkman 1976 and Irwin 1980, Chapter 6). Furthermore, 
using the same sort of redefinition of generations and periods that we use to transform an economy with consumers who live for many periods into one in which they live for two, we can formally transform an economy with a $\mathrm{k}$ cycle, $\left(\mathrm{p}^{1}, \mathrm{p}^{2}, \ldots, \mathrm{p}^{\mathrm{k}}\right)$, into an economy with $\mathrm{n} \times \mathrm{k}$ goods in each period in which this cycle is a steady state. What we are missing therefore is a study of chaotic equilibria that converge neither to steady states nor to cycles. See Benhabib and Day (1982) and Grandmont (1985) for examples of such equilibria.

There are two types of steady states, those in which there is fiat money and those in which there is not. Using Walras's law and the equilibrium conditions, we can show that

$$
\mathrm{p}_{1}^{\prime} \mathrm{z}_{0}\left(\mathrm{p}_{1}\right)=-\mathrm{p}_{1}^{\prime} \mathrm{y}\left(\mathrm{p}_{1}, \mathrm{p}_{2}\right)=\mathrm{p}_{2}^{\prime} \mathrm{z}\left(\mathrm{p}_{1}, \mathrm{p}_{2}\right)=\ldots=-\mathrm{p}_{\mathrm{t}}^{\prime} \mathrm{y}\left(\mathrm{p}_{\mathrm{t}}, \mathrm{p}_{\mathrm{t}+1}\right)
$$

Let $\mathrm{M}=-\mathrm{p}^{\prime} \mathrm{y}(\mathrm{p}, \beta \mathrm{p})$ Walras's law, $\mathrm{p}^{\prime} \mathrm{y}(\mathrm{p}, \beta \mathrm{p})+\beta \mathrm{p}^{\prime} \mathrm{z}(\mathrm{p}, \beta \mathrm{p})=0$, and the steady state condition imply that $(\beta-1) p^{\prime} y(p, \beta)=0$; that is, $\beta=1$ or $M=0$. Kehoe and Levine (1984) prove that these two possibilities almost never occur simultaneously. Steady states in which $\beta=1$ and $\mathrm{M} \neq 0$ are called monetary steady states; those in which $\beta \neq 0$ and $M=0$ are called real steady states. Proving the existence of, and computing, steady states of each type is similar to doing the same for static exchange economies. Monetary steady states are given by a price vector $p \in R_{+}^{\mathrm{n}} \backslash\{0\}$ that satisfies

$$
f(p)=z(p, p)+y(p, p)=0 .
$$

Here the function $f$ has all of the properties of the excess demand function of a static exchange economy. Real steady states are given by a pair $(p, \beta)$ that satisfies

$$
\begin{aligned}
& z(p, \beta p)+y(p, \beta p)=0 \\
& p^{\prime} y(p, \beta p)=0
\end{aligned}
$$

Using arguments similar to those in Section 2, Kehoe and Levine (1984) characterize the solution to this system of equations as the solution to a fixed point problem in $\mathrm{R}^{\mathrm{n}+1}$. As well as proving the 
existence of each type of steady state, they argue that there is almost always a finite number of each type, and they provide index theorems that imply uniqueness conditions for each type.

Let us now study the question of how many equilibria converge to the steady state $(p, \beta p)$ by linearizing the equilibrium conditions. Making use of the homogeneity of $y$ and $z$, which implies that their derivatives are homogeneous of degree minus one and that

$$
\mathrm{D}_{1} \mathrm{yp}+\beta \mathrm{D}_{2} \mathrm{yp}=\mathrm{D}_{1} \mathrm{zp}+\beta \mathrm{D}_{2} \mathrm{zp}=0
$$

we write the linearized system as

$$
\begin{aligned}
& \mathrm{D}_{2} \mathrm{yp}+\left(\mathrm{D}_{1} \mathrm{y}+\mathrm{Dz} \mathrm{z}_{0}\right) \mathrm{p}_{1}=\mathrm{Dz} \mathrm{z}_{0} \mathrm{p}-\mathrm{z}_{0}(\mathrm{p})-\mathrm{y}(\mathrm{p}, \beta \mathrm{p}) \\
& \mathrm{D}_{2} \mathrm{yp}_{\mathrm{t}+1}+\left(\mathrm{D}_{1} \mathrm{y}+\beta \mathrm{D}_{2} \mathrm{z}\right) \mathrm{p}_{\mathrm{t}}+\beta \mathrm{D}_{1} \mathrm{zp}_{\mathrm{t}-1}=0, \quad \mathrm{t}=2,3, \ldots
\end{aligned}
$$

Here $D_{2} y$ is, for example, the $n \times n$ matrix of partial derivatives of $y$ with respect to its second vector of arguments evaluated at $(p, \beta p)$.

We can write the equilibrium conditions in periods $t=2,3, \ldots$ as the first order system $\mathrm{q}_{\mathrm{t}}=\mathrm{Gq}_{\mathrm{t}-1}$ where $\mathrm{q}_{\mathrm{t}}=\left(\mathrm{p}_{\mathrm{t}}, \mathrm{p}_{\mathrm{t}+1}\right)$ and

$$
G=\left[\begin{array}{cc}
0 & I \\
-\beta D_{2} y^{-1} D_{1} z & -D_{2} y^{-1}\left(D_{1} y+\beta D_{2} z\right)
\end{array}\right]
$$

As in the linear-quadratic model of Section 5.5, the stability properties of this difference equation are governed by the eigenvalues of $\mathrm{G}$. The homogeneity of $\mathrm{y}$ and $\mathrm{z}$ imply that $\beta$ is an eigenvalue since

$$
\mathrm{G}\left[\begin{array}{c}
\mathrm{p} \\
\beta \mathrm{p}
\end{array}\right]=\left[\begin{array}{c}
\beta \mathrm{p} \\
\beta^{2} \mathrm{p}
\end{array}\right]
$$

Differentiating Walras's law and evaluating the result at $\left(\mathrm{p}_{\mathrm{t}}, \mathrm{p}_{\mathrm{t}+1}\right)=(\mathrm{p}, \beta \mathrm{p})$ implies that

$$
\begin{aligned}
& y^{\prime}+p^{\prime} D_{1} y+\beta p^{\prime} D_{1} z=0 \\
& z^{\prime}+p^{\prime} D_{2} y+\beta p^{\prime} D_{2} z=0
\end{aligned}
$$


Consequently, 1 is another eigenvalue since

$$
\left[\beta \mathrm{p}^{\prime} \mathrm{D}_{1} \mathrm{z}-\mathrm{p}^{\prime} \mathrm{D}_{2} \mathrm{y}\right] \mathrm{G}=\left[\beta \mathrm{p}^{\prime} \mathrm{D}_{1} \mathrm{z}-\mathrm{p}^{\prime} \mathrm{D}_{2} \mathrm{y}\right] .
$$

In the case where $\beta=1$, these are generally the same restriction and we have information only about one eigenvalue.

Again solutions to the difference equation $\mathrm{q}_{\mathrm{t}}=\mathrm{Gq}_{\mathrm{t}-1}$ take the form

$$
\left[\begin{array}{c}
p_{t} \\
p_{t+1}
\end{array}\right]=\sum_{i=1}^{2 n} c_{i} \lambda_{i}^{t-1}\left[\begin{array}{c}
z_{i} \\
\lambda_{i} z_{i}
\end{array}\right]
$$

where the (possibly) complex constants $\mathrm{c}_{\mathrm{i}}$ are determined by the initial conditions

$$
\left[\begin{array}{l}
\mathrm{p}_{1} \\
\mathrm{p}_{2}
\end{array}\right]=\sum_{\mathrm{i}=1}^{\mathrm{n} n} \mathrm{c}_{\mathrm{i}}\left[\begin{array}{c}
\mathrm{z}_{\mathrm{i}} \\
\lambda_{\mathrm{i}} \mathrm{z}_{\mathrm{i}}
\end{array}\right] .
$$

To ensure convergence to the steady state $(p, \beta p)$ we need to put positive weight on the eigenvector $(p, \beta p)$ and zero weights on eigenvectors $\left(z_{i}, \lambda_{i} z_{i}\right)$ for which the modulus $\left|\lambda_{i}\right|$ is greater than $\beta$.

The situation is in many ways similar to that in the linear-quadratic model of Section 5 . In place of the $n_{k}$ initial conditions $k_{1}$ of the $2 n_{k}$ conditions $\left(k_{1}, k_{2}\right)$ that we need to start up the difference equation, we have $\mathrm{n}$ restrictions

$$
\mathrm{D}_{2} \mathrm{yp}+\left(\mathrm{D}_{1} \mathrm{y}+\mathrm{Dz} \mathrm{z}_{0}\right) \mathrm{p}_{1}=\mathrm{Dz}_{0} \mathrm{p}-\mathrm{z}_{0}(\mathrm{p})-\mathrm{y}(\mathrm{p}, \beta \mathrm{p})
$$

on the $2 n$ required initial conditions $\left(p_{1}, p_{2}\right)$. Unlike the linear-quadratic model, however, there is no need for there to be fewer than $n$ stable eigenvalues of $G$.

By counting the number of eigenvalues of $\mathrm{G}$ less than $\beta$ in modulus, we can determine the dimension of the stable manifold, the sets of points $\left(\mathrm{p}_{1}, \mathrm{p}_{2}\right) \in \mathrm{R}^{2 \mathrm{n}}$ that serve as starting values for the implicit difference equation generated by the equilibrium conditions in periods $t=2,3, \ldots$, and converge to the steady state $(p, \beta \rho)$. If we then subtract the number of restrictions on $\left(\mathrm{p}_{1}, \mathrm{p}_{2}\right)$ implied by the equilibrium conditions in the first period, we can, in general, determine the dimension of the manifold of equilibria that converge to the steady state. We need to distinguish between two cases, 
economies without fiat money and economies with fiat money. The distinction is crucial because Walras's law and the equilibrium conditions in the first period imply that

$$
\left[\beta p^{\prime} D_{1} z-p^{\prime} D_{2} y\right]\left[\begin{array}{l}
p_{1} \\
p_{2}
\end{array}\right]=\sum_{i=1}^{m_{0}} M_{i}
$$

This, in turn, implies that the eigenvector associated with the eigenvalue $\lambda=1$ must receive zero weight $c_{i}$ in the initial conditions if $\sum_{i=1}^{m_{0}} M_{i}=0$ and must receive nonzero weight if $\sum_{i=1}^{m_{0}} M_{i} \not \neq 0$. (See Kehoe and Levine $1985,1990 \mathrm{~b}$ for details.) In the case where there is fiat money, the dimension of the set of equilibria is generically $n^{\$}+1-n$, where $n^{s}$ is the number of eigenvalues with $|\lambda|<\beta$; there is one eigenvalue $\lambda=\beta$, and $n$ is the number of restrictions implied by the initial conditions. This number can be as large as $n$. In the case where there is no fiat money, the dimension of the set of equilibria is generically $\bar{n}^{s}+1-n$, where $\bar{n}^{\mathbf{s}}$ is the number of eigenvalues with $|\lambda|<\beta$ excluding, if need be, the eigenvalue $\lambda=1$. This number can be as large as $n-1$ and is equal to zero when there is saddle point splitting, when half of the $2 n-2$ eigenvalues of $G$, not counting $\lambda=1$ or $\beta$, are less than $\beta$ in modulus and the other half are greater.

Another possibility, of course, is that there are too few stable eigenvalues. In this case, for almost all initial generations $\mathrm{z}_{0}$, there is no equilibrium that converges to the steady state. In this case we call the steady state unstable.

Example 6.1. Consider an overlapping generations in which generation $t, t=1,2, \ldots$, contains a single consumer who lives for three periods. There is one good in every period. Using the procedure described in Section 6.1, we could convert this to an economy with two consumers who live for two periods in each generation and two goods in each period. The consumer born in period $t$ has the utility function 


$$
u\left(x_{t}, x_{t+1}, x_{t+2}\right)=\sum_{j=1}^{3} a^{j-1}\left(x_{t+j-1}^{b}-1\right) / b
$$

where $\mathrm{a}>0$ and $\mathrm{b}<1$. Given an endowment stream $\left(\mathrm{w}_{1}, \mathrm{w}_{2}, \mathrm{w}_{3}\right)$, the consumer maximizes this utility function subject to the constraint

$$
\sum_{j=1}^{3} p_{t+j-1} x_{t+j-1} \leq \sum_{j=1}^{3} p_{t+j-1} w_{j}
$$

The consumer's excess demand functions are

$$
\mathbf{z}_{j}\left(\mathrm{p}_{\mathrm{t}}, \mathrm{p}_{\mathrm{t}+1}, \mathrm{p}_{\mathrm{t}+2}\right)=\frac{\gamma_{\mathrm{j}} \sum_{\mathrm{k}=1}^{3} \mathrm{p}_{\mathrm{t}+\mathrm{k}-1} \mathrm{w}_{\mathrm{k}}}{\mathrm{p}_{\mathrm{t}+\mathrm{j}-1}^{\eta} \sum_{\mathrm{k}=1}^{3} \gamma_{\mathrm{k}} \mathrm{p}_{\mathrm{t}+\mathrm{k}-1}^{\mathrm{I}-\eta}}-\mathrm{w}_{\mathrm{j}}, \quad \mathrm{j}=1,2,3 .
$$

Here $\eta=1 /(1-b)$ and $\gamma_{j}=a^{(j-1) \eta}, j=1,2,3$.

In addition to these consumers, there are two others, an old consumer who lives only in period 1 and a middle-aged consumer who lives in periods 1 and 2 . The old consumer, consumer -1 , derives utility only from consumption of the single good in the first period, so we need not specify a utility function. If he has $\mathrm{M}_{-1}$ units of flat money, which may be positive, negative, or zero, his excess demand function is

$$
z_{3}^{-1}\left(p_{1}\right)=M_{-1} / p_{1}
$$

The middle-aged consumer, consumer 0 has the utility function

$$
u_{0}\left(x_{2}, x_{3}\right)=\sum_{j=1}^{2} a^{j}\left(x_{j+1}^{b}-1\right) / b,
$$

an endowment stream of goods, $\left(w_{2}^{0}, w_{3}^{0}\right)$, and an endowment of fiat money, $M_{0}$. His excess demand functions are 


$$
z_{j}^{0}\left(p_{1}, p_{2}\right)=\frac{\gamma_{j}\left(\sum_{k=1}^{2} p_{k} w_{k+1}^{0}+M_{0}\right)}{p_{j-1}^{\eta} \sum_{k=1}^{2} \gamma_{k+1} p_{k}^{1-\eta}}-w_{j}^{0}, \quad j=2,3 .
$$

The equilibrium conditions for this economy are

$$
\begin{aligned}
& \mathrm{z}_{3}^{-1}\left(\mathrm{p}_{1}\right)+\mathrm{z}_{2}^{0}\left(\mathrm{p}_{1}, \mathrm{p}_{2}\right)+\mathrm{z}_{1}\left(\mathrm{p}_{1}, \mathrm{p}_{2}, \mathrm{p}_{3}\right)=0 \\
& \mathrm{z}_{3}^{0}\left(\mathrm{p}_{1}, \mathrm{p}_{2}\right)+\mathrm{z}_{2}\left(\mathrm{p}_{1}, \mathrm{p}_{2}, \mathrm{p}_{3}\right)+\mathrm{z}_{1}\left(\mathrm{p}_{2}, \mathrm{P}_{3}, \mathrm{p}_{4}\right)=0 \\
& \mathrm{z}_{3}\left(\mathrm{p}_{\mathrm{t}-2}, \mathrm{P}_{\mathrm{t}-1}, \mathrm{p}_{\mathrm{t}}\right)+\mathrm{z}_{2}\left(\mathrm{P}_{\mathrm{t}-1}, \mathrm{p}_{\mathrm{t}}, \mathrm{p}_{\mathrm{t}+1}\right)+\mathrm{z}_{1}\left(\mathrm{p}_{\mathrm{t}}, \mathrm{p}_{\mathrm{t}+1}, \mathrm{p}_{\mathrm{t}+2}\right)=0, \quad \mathrm{t}=3,4
\end{aligned}
$$

The equilibrium condition for period $t, t=3,4, \ldots$, can be linearized at a steady state $\left(1, \beta, \beta^{2}\right)$ as

$$
\begin{aligned}
\beta^{2} \mathrm{D}_{1} \mathrm{z}_{3} \mathrm{p}_{\mathrm{t}-2} & +\left(\beta^{2} \mathrm{D}_{2} \mathrm{z}_{3}+\beta \mathrm{D}_{1} \mathrm{z}_{2}\right) \mathrm{p}_{\mathrm{t}-1}+\left(\beta^{2} \mathrm{D}_{3} \mathrm{z}_{3}+\beta \mathrm{D}_{2} \mathrm{z}_{2}+\mathrm{D}_{1} \mathrm{z}_{1}\right) \mathrm{p}_{\mathrm{t}} \\
& +\left(\beta \mathrm{D}_{3} \mathrm{z}_{2}+\mathrm{D}_{2} \mathrm{z}_{1}\right) \mathrm{p}_{\mathrm{t}+1}+\mathrm{D}_{3} \mathrm{z}_{1} \mathrm{p}_{\mathrm{t}+2}=0
\end{aligned}
$$

Here all derivatives are evaluated at the $\left(1, \beta, \beta^{2}\right)$. Corresponding to the condition that $D_{2} y$ is nonsingular in the general two-period-lived case is the condition that $\mathrm{D}_{3} \mathrm{z}_{1}$ is nonzero. Corresponding to the eigenvalues of $\mathrm{G}$ are the roots of the fourth order polynomial

$$
\begin{aligned}
\beta^{2} \mathrm{D}_{1} \mathrm{z}_{3} & +\left(\beta^{2} \mathrm{D}_{2} \mathrm{z}_{3}+\beta \mathrm{D}_{1} \mathrm{z}_{2}\right) \lambda+\left(\beta^{2} \mathrm{D}_{3} \mathrm{z}_{3}+\beta \mathrm{D}_{2} \mathrm{z}_{2}+\mathrm{D}_{1} \mathrm{z}_{1}\right) \lambda^{2} \\
& +\left(\beta \mathrm{D}_{3} \mathrm{z}_{2}+\mathrm{D}_{2} \mathrm{z}_{1}\right) \lambda^{3}+\mathrm{D}_{3} \mathrm{z}_{1} \lambda^{4}=0 .
\end{aligned}
$$

The consumer has $\mathrm{a}=1 / 2, \mathrm{~b}=-4$, and $\left(\mathrm{w}_{1}, \mathrm{w}_{2}, \mathrm{w}_{3}\right)=(5,20,1)$. To calculate the roots of the fourth order polynomial, which determine the determinacy properties of equilibria near a steady state, we start by evaluating the derivatives of excess demand at $\left(\mathrm{p}_{t}, \mathrm{p}_{\mathrm{t}+1}, \mathrm{p}_{\mathrm{t}+2}\right)=\left(1, \beta, \beta^{2}\right)$. At $\beta=1$, for example, these derivatives are

$$
\left[\begin{array}{lll}
\mathrm{D}_{1} \mathrm{z}_{1} & \mathrm{D}_{2} \mathrm{z}_{1} & \mathrm{D}_{3} \mathrm{z}_{1} \\
\mathrm{D}_{1} \mathrm{z}_{2} & \mathrm{D}_{2} \mathrm{z}_{2} & \mathrm{D}_{3} \mathrm{z}_{2} \\
\mathrm{D}_{1} \mathrm{z}_{3} & \mathrm{D}_{2} \mathrm{z}_{3} & \mathrm{D}_{3} \mathrm{z}_{3}
\end{array}\right]=\left[\begin{array}{lll}
-3.0869 & 4.9881 & -1.9013 \\
-0.9650 & 2.6201 & -1.6551 \\
-0.8401 & 3.7803 & -2.9402
\end{array}\right] \text {. }
$$


(Notice that, since this matrix has some negative off-diagonal elements, $\left(z_{1}, z_{2}, z_{3}\right)$ violates gross substitutability.) The polynomial that we are interested in is

$$
-0.8401+2.8153 \lambda-3.4070 \lambda^{2}+3.3330 \lambda^{3}-1.9013 \lambda^{4}=0 .
$$

The four roots are $\lambda=1,0.4860,0.1335+0.9441 \mathrm{i}$, and $0.1335-0.9441 \mathrm{i}$ as can easily be verified. This example has four steady states. The steady states and the corresponding roots are listed below:

\begin{tabular}{|lllll|}
\hline$\beta$ & & \multicolumn{3}{c|}{ Other Roots } \\
\hline 0.0976 & 1 & -0.0102 & & 0.3845 \\
0.4286 & 1 & & $0.1275 \pm 0.3337 \mathrm{i}$ & \\
1 & 0.4861 & & $0.1335 \pm 0.9441 \mathrm{i}$ & \\
903.6581 & 1 & -1196.3574 & & 2.2889 \\
\hline
\end{tabular}

At the steady state $\beta=0.4286$ the modulus of the pair of complex conjugates is 0.3572 ; at $\beta=1$ it is 0.9535 .

To generate examples with multiplicity of equilibria, we can choose the initial two consumers, generations -1 and 0 , so that $\left(1, \beta, \beta^{2}, \beta^{3}\right)$ satisfies the equilibrium conditions in the first two periods. When $\beta=0.4286$, we can generate an example without fiat money that has a one dimensional manifold of equilibria since $\bar{n}^{s}=2$ and $\bar{n}^{s}+1-n=1$. (To do so we set $\left(w_{2}^{0}, w_{3}^{0}\right)=$ $(12.0650,1)$.) When $\beta=1$, we can generate an example with fiat money that has a two dimensional manifold of equilibria since $n^{8}=3$ and $n^{s}+1-n=2$. (See Kehoe and Levine $1990 \mathrm{~b}$ for details.)

A similar analysis of the possibility of indeterminacy in economies with production and a mixture of overlapping generations and infinitely lived consumers is presented by Muller and 
Woodford (1988). Similar analyses for nonstationary economies, economies in which the functions $y_{t}$ and $z_{t}$ vary over time, have been presented by Geanakoplos and Brown (1985) and Santos and Bona (1989).

\subsection{Computational Methods}

There are two alternatives for approximating an equilibrium of an infinite horizon overlapping generations model: The first is to linearize the equilibrium conditions around a steady state (or cycle) and then solve the linearized model (see Laitner 1984, 1990 and Kehoe and Levine $1985,1990 \mathrm{~b})$. The second is to truncate the model and to compute an equilibrium of the truncated model. The crucial question is, What terminal conditions should we impose? A popular choice is to impose the condition that the equilibrium converges to a given steady state (see, for example, Auerbach and Kotlikoff 1987). Care must be taken in this case to make sure that the truncated economy as a whole satisfies Walras's law.

The two methods proposed above are probably best viewed as complements rather than substitutes. Indeed, before computing a truncated equilibrium using as terminal conditions the requirement that the equilibrium converge to a given steady state, we should linearize the model around that steady state. This would allow us to check for indeterminacy, where a continuum of equilibria converge to the steady state, or instability, where no equilibrium converges to it. We would not want to impose the condition of convergence to an unstable steady state in a truncated model. Indeterminacy is more problematical, but we would certainly want to be aware of it since it indicates that the equilibrium that is computed is acutely sensitive to the truncation date $T$ and the exact form of the terminal conditions. Figure 6.1 depicts three different equilibria of the economy in Example 6.1, each of which converges to the steady state $\beta=0.4286$. Notice how small 


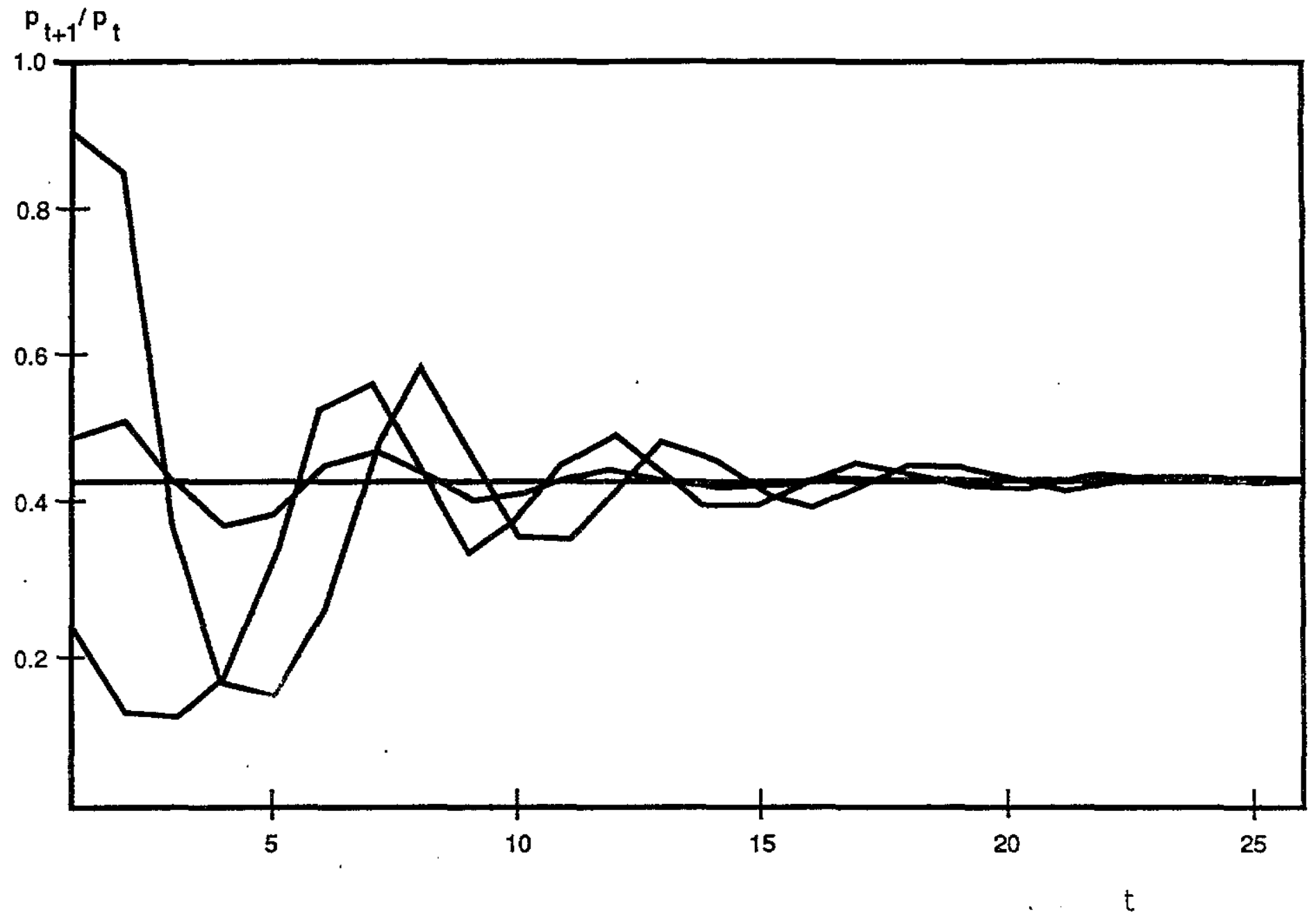

FIGURE 6.1 
differences in the equilibrium paths at $\mathrm{T}=20$ correspond to large differences earlier in the path. (See Kehoe and Levine $1990 \mathrm{~b}$ for a discussion.)

To compute an equilibrium of a truncated model, we could use the methods developed for static economies. The truncated model may be very large, however, and it is often more convenient to view the problem of computing an equilibrium as a two-point boundary-value problem. Auerbach and Kotlikoff (1987) and Lipton, Poterba, Sachs, and Summers (1982) have proposed alternative methods for solving this sort of problem. The equilibrium condition in period $t, t=2,3, \ldots$, $\mathrm{T}-1$, implicitly defines a system of $\mathrm{n}$ second order nonlinear difference equations. The equilibrium conditions in period 1 implicitly provide $n$ of the $2 n$ necessary boundary conditions. The equilibrium conditions in period $\mathrm{T}$ implicitly provide the other $\mathrm{n}$. Auerbach and Kotlikoff (1987) solve this problem using a nonlinear Gauss-Seidel method. They start by guessing a solution, the steady state for instance. They then solve the model going forward using this guess as expectations for future variables. After they are done, they use the calculated solution as a new guess and repeat the process. They stop when, and if, the calculated solution agrees with the previous guess.

We write the equilibrium conditions in period $t$ as

$$
f\left(p_{t-1}, p_{t}, p_{t+1}\right)=z\left(p_{t-1}, p_{t}\right)+y\left(p_{t}, p_{t+1}\right)=0
$$

Suppose that we are interested in equilibria that converge to the steady state $(p, \beta p)$. We perform a change of variables, deflating prices in each period by the factor $\beta$, so that $\tilde{p}_{t}=\beta^{-t_{1}}$ and

$$
\tilde{\mathrm{f}}\left(\tilde{\mathrm{p}}_{\mathrm{t}-1}, \tilde{\mathrm{p}}_{\mathrm{t}}, \tilde{\mathrm{p}}_{\mathrm{t}+1}\right)=\mathrm{f}\left(\tilde{\mathrm{p}}_{\mathrm{t}-1}, \beta \tilde{\mathrm{p}}_{\mathrm{t}}, \beta^{2} \tilde{\mathrm{p}}_{\mathrm{t}+1}\right)=\mathrm{f}\left(\mathrm{p}_{\mathrm{t}-1}, \mathrm{p}_{\mathrm{t}}, \mathrm{p}_{\mathrm{t}+1}\right)
$$

We henceforth write $p_{t}$ for $\tilde{p}_{t}$ and $f$ for $\tilde{\mathrm{f}}$.

Given the guess $p_{1}^{k}, \ldots, p_{T}^{k}$, the nonlinear Gauss-Seidel method sets $p_{t}^{k+1}=g_{t}\left(p^{k}\right)$ in the system

$$
\mathrm{f}_{1}\left(\mathrm{~g}_{1}\left(\mathrm{p}^{\mathrm{k}}\right), \mathrm{p}_{2}^{\mathrm{k}}\right)=0
$$




$$
\begin{aligned}
& \mathrm{f}\left(\mathrm{g}_{\mathrm{t}-1}\left(\mathrm{p}^{\mathrm{k}}\right), \mathrm{g}_{\mathrm{t}}\left(\mathrm{p}^{\mathrm{k}}\right), \mathrm{p}_{\mathrm{t}+1}^{\mathrm{k}}\right)=0, \mathrm{t}=2, \ldots, \mathrm{T}-1 \\
& \mathrm{f}_{\mathrm{T}}\left(\mathrm{g}_{\mathrm{T}-1}\left(\mathrm{p}^{\mathrm{k}}\right), \mathrm{g}_{\mathrm{t}}\left(\mathrm{p}^{\mathrm{k}}\right)\right)=0 .
\end{aligned}
$$

Near the equilibrium $\hat{\mathrm{p}}_{1}, \hat{\mathrm{p}}_{2}, \ldots, \hat{\mathrm{p}}_{\mathrm{T}}$, this algorithm converges to the linear Gauss-Seidel method for solving

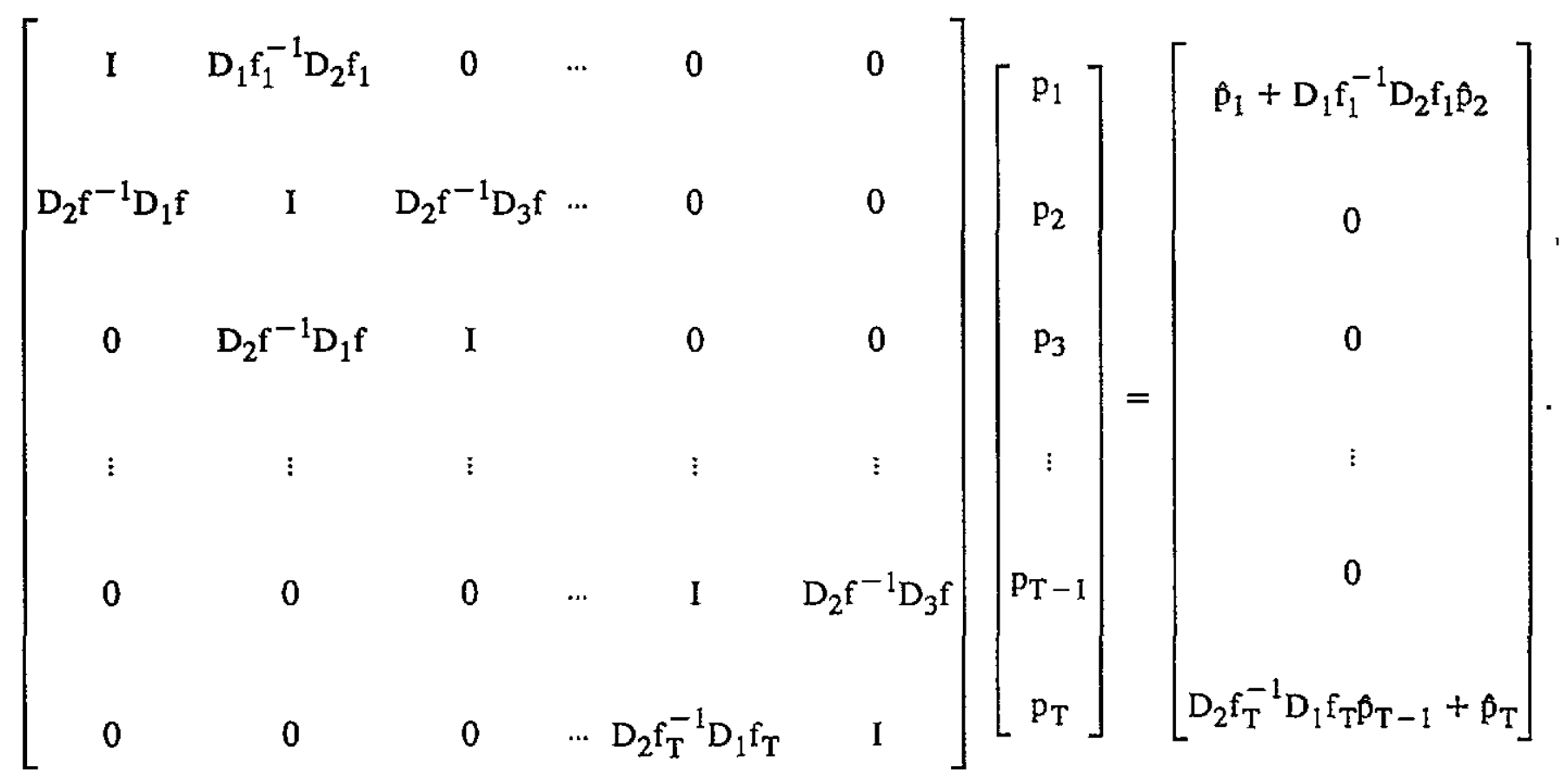

(Depending on whether there is no fiat money, we may need to normalize $\hat{\rho}_{11}=0$ and delete one equation in the first period.) In this system of equations the derivatives are evaluated at $\hat{p}_{1}, \hat{p}_{2}, \ldots$, $\mathrm{\rho}_{\mathrm{T}}$ rather than at the steady state $\mathrm{p}$. Notice, however, that, if the equilibrium of the infinite horizon model converges to $\mathrm{p}$, then as we move down the matrix the derivatives approach those at the steady state.

The Gauss-Seidel method splits the coefficient matrix of this system, which we write as

$$
\mathrm{Ap}=\mathrm{c},
$$

into a strictly upper-triangular component $\mathrm{U}$, a strictly lower-triangular component $\mathrm{L}$, and a diagonal component $\mathrm{D}$. We then solve 


$$
(\mathrm{D}+\mathrm{L}) \mathrm{p}^{\mathrm{k}+1}=-\mathrm{Up}^{\mathrm{k}}+\mathrm{c}
$$

recursively. This method converges if and only if the eigenvalues of $-(D+L)^{-1} U$ are less than one in modulus. Young (1971) provides conditions for convergence. It can be shown, for example, that, if $f$ exhibits gross substitutability and there is no fiat money, the method converges. (Compare with Kehoe, Levine, Mas-ColelI, and Woodford 1991.)

The speed of convergence is $-\log |\lambda|$ where $\lambda$ is the eigenvalue of $-(D+L)^{-1} U$ with the largest modulus. If the infinite horizon model exhibits indeterminacy, then the matrix $A$ is almost singular and $-\log |\lambda|$ is close to zero. To see this, notice that there would then be two vectors $\mathrm{p}^{1} \neq \mathrm{p}^{2}$ such that, for $\mathrm{T}$ large,

$$
\begin{aligned}
& A p^{1}=A p^{2} \approx c \\
& A\left(p^{1}-p^{2}\right) \approx 0 .
\end{aligned}
$$

A slight perturbation in A would then make it singular. Standard results in linear algebra then imply that $A$ has an eigenvalue very close to zero and, therefore, that $-(D+L)^{-1} U$ has an eigenvalue very close to to one. Similarly, instability in the infinite horizon model, where no solution converges to the steady state, also corresponds to A being almost singular. This is because, while indeterminacy corresponds to the infinite version of A not being one-to-one, instability corresponds to it not being onto (see Kehoe, Levine, Mas-Colell, and Zame 1989).

This means that the Gauss-Seidel method does not work if there is indeterminacy or instability: One possibility is that convergence may be impossibly slow. If there is indeterminacy, another possibility is that the algorithm may stop at a point far from the true equilibrium of the truncated model but close to an equilibrium of the infinite horizon model. If there is instability, in contrast, the algorithm may stop at a point far from either. In either case, a crucial factor determining the results is the convergence criterion, the degree to which the equilibrium conditions must be satisfied for the algorithm to stop. (See Kehoe and Levine 1990a.) 
Our analysis suggests directions for further research: One way to speed up the Auerbach-Kotlikoff algorithm would be to linearize the equilibrium conditions around a guess and then solve, not using Gauss-Seidel, but a successive overrelaxation method,

$$
(\mathrm{D}+\alpha \mathrm{L}) \mathrm{p}^{\mathrm{k}+1}=((1-\alpha)-\alpha \mathrm{U}) \mathrm{p}^{\mathrm{k}}+\alpha \mathrm{c},
$$

for a good choice of $\alpha$ (see Young 1971). The system can then be linearized around the new guess and the procedure repeated. This method is just Newton's method with a fast way of inverting the sparse matrix A. Unfortunately, of course, it does not work well when A is almost singular or when $-(\mathrm{D}+\mathrm{L})^{-1} \mathrm{U}$ has an eigenvalue greater than one in modulus.

An alternative method for solving this sort of equilibrium problem has been suggested by Lipton, Poterba, Sachs, and Summers (1982). This is a method widely used by engineers and physical scientists called multiple shooting (see, for example, Keller 1968). They start by guessing the initial values for prices and solving forward for the resulting price path up to $T$ :

$$
\begin{aligned}
& f_{1}\left(p_{1}, p_{2}\left(p_{1}\right)\right)=0 \\
& f\left(p_{t-1}\left(p_{1}\right), p_{t}\left(p_{1}\right), p_{t+1}\left(p_{1}\right)\right)=0, t=2, \ldots, T .
\end{aligned}
$$

They then apply Newton's method to solve $\mathrm{p}_{\mathrm{T}}\left(\mathrm{p}_{1}\right)-\left\|\mathrm{p}_{\mathrm{T}}\left(\mathrm{p}_{1}\right)\right\| \beta \mathrm{p}=0$, where $(\mathrm{p}, \beta \mathrm{p})$ is the steady state. Unless the equilibria are completely indeterminate, however, most paths diverge very rapidly. Because of this the algorithm is very numerically unstable. They therefore propose dividing the time period into difference time segments, then solving

$$
\begin{aligned}
& \mathrm{p}_{\mathrm{T}_{1}}\left(\mathrm{p}_{1}\right)-\mathrm{p}_{\mathrm{T}_{1}}=0 \\
& \mathrm{p}_{\mathrm{T}_{2}}\left(\mathrm{p}_{\mathrm{T}_{1:}}\right)-\mathrm{p}_{\mathrm{T}_{2}}=0 \\
& \mathrm{p}_{\mathrm{T}_{\mathrm{m}}}\left(\mathrm{p}_{\mathrm{T}_{\mathrm{m}-1}}\right)-\left\|\mathrm{p}_{\mathrm{T}_{\mathrm{m}}}\left(\mathrm{p}_{\mathrm{T}_{\mathrm{m}-1}}\right)\right\| \beta \mathrm{p}=0
\end{aligned}
$$

for $\mathrm{p}_{1}, \mathrm{p}_{\mathrm{T}_{1}}, \ldots, \mathrm{p}_{\mathrm{T}_{\mathrm{m}-1}}$ where $\mathrm{m}$ is the number of time segments. 
Unfortunately, this method is not a good one for many economic problems for at least three related reasons: First, it is often difficult to solve $f\left(p_{t-1}, p_{t}, p_{t+1}\right)=0$ for $p_{t+1}$ as a function of $\left(p_{t-1}, p_{t}\right)$. There may be multiple solutions (this, however, is a problem with most methods). Furthermore, any small divergence from the true solution for $\left(p_{t-1}, p_{t}\right)$ can cause $p_{t+1}$ to become negative or not to exist. Second, dividing the time horizon into segments causes the number of variables in Newton's method to go up rapidly. In Auerbach and Kotlikoff's model the number of variables in what corresponds to $\mathrm{p}_{1}$ is 54 and $\mathrm{T}$ is 150 . Dividing into three time segments produces $3 \times 54$ variables, which is greater than the original number of variables in the system, 150 . Even dividing into three segments is probably inadequate, however, since, as Laitner (1990) reports, the linearization of Auerbach and Kotlikoff's model has unstable eigenvalues 1.6 times as large in modulus as the largest stable eigenvalue. This implies that almost all small errors in guessing $p_{1}$ have a nonzero complement that is blown up by a factor of $1.6^{50}$, which has order of magnitude $10^{10}$, in $\mathrm{p}_{\mathrm{T}_{1}}\left(\mathrm{p}_{1}\right)$. Third, and most importantly, shooting methods work well on ordinary two-point boundary-value problems, not saddle-point problems. In fact, it is exactly this sort of problem for which Press, Flannery, Teukolsky, and Vetterling (1986, pp. 580-81) advise that shooting methods should not be used.

Kehoe and Levine (1990a) suggest several methods for computing approximate equilibria that combine the advantages of solving the linearized model with those of solving the truncated model. The simplest is to replace the terminal condition of convergence to a steady state by date $\mathrm{T}$ with the condition of convergence to the stable subspace of the linearized equilibrium conditions. When the equilibrium path is close to the steady state, this subspace is a very good approximation to the stable manifold of the nonlinear system defined by the original equilibrium conditions.

All of the methods proposed in this section are applicable to nonstationary economies that are stationary after some date. Extensions to economies with uncertainty seem much more difficult, 
however; see Duffie, Geanakoplos, Mas-Colell, and McLennan (1988) for a discussion of some of the problems involved in even proving the existence of equilibria with the sort of stationarity properties that make them tractable.

\section{Economies with Taxes and Externalities}

In applications many economies fail to satisfy the conditions of the two welfare theorems because of such features as distortionary taxes, externalities, rationing, and institutionally fixed prices.

\subsection{Static Economies with Taxes}

Large scale general equilibrium models with taxes and government spending are frequently used in policy analysis; see Shoven and Whalley (1984) for a survey. An early approach to proving the existence of, and computing, equilibrium of such models is given by Shoven and Whalley (1973). Here, as do Todd (1979) and Kehoe (1985a), we follow an approach similar to that in Sections 2.1 and 4.1 for proving the existence of equilibrium in static economies without taxes.

Consider an economy similar to that in Section 4 . Consumer $i$ now faces the budget constraint $\sum_{\mathrm{j}=1}^{\mathrm{n}} \mathrm{p}_{\mathrm{j}}\left(1+\tau_{\mathrm{j}}\right) \mathrm{x}_{\mathrm{j}} \leq \mathrm{p}^{\prime} \mathrm{w}^{\mathrm{i}}+\theta_{\mathrm{i}} \mathrm{r}$ where $\tau_{\mathrm{j}}$ is the ad valorem tax on good $\mathrm{j}$ and $\theta_{\mathrm{i}}, \theta_{\mathrm{i}} \geq 0$, $\sum_{\mathrm{i}=1}^{\mathrm{m}} \theta_{\mathrm{i}}=1$, is the share of government revenue $\mathrm{r}$ received by consumer $\mathrm{i}$. In many applications $\theta_{1}=1$ while $\theta_{2}=\ldots=\theta_{\mathrm{m}}=0$; that is, the first consumer is the government.

The responses of the consumers to a vector of prices and a level of government revenue can be aggregated into an aggregate excess demand function $f:\left(R_{+}^{n} \backslash\{0\}\right) \times R_{+} \rightarrow R^{n}$ and a tax function $\mathrm{t}:\left(\mathbf{R}_{+}^{\mathbf{n}} \backslash\{0\}\right) \times \mathbf{R}_{+} \rightarrow \mathrm{R}_{+}$,

$$
t(p, r)=\sum_{j=1}^{n} p_{j} \tau_{j} \sum_{i=1}^{m} x_{j}^{i}(p, r)
$$


Both $f$ and $t$ are continuous. $f$ is also homogeneous of degree zero, is bounded below by $-\sum_{i=1}^{m} w^{i}$, and satisfies $\left\|f\left(p, r^{l}\right)\right\| \rightarrow \infty$ as $r^{\ell} \rightarrow 1$ for any $p \in R_{+}^{n} \backslash\{0\}, \quad t$ is homogeneous of degree one. Together $f$ and $t$ satisfy a modified version of Walras's law, $p^{\prime} f(p, r)+t(p, r)=r$. Analogously with the model without taxes, we can specify the consumers either in terms of utility functions and endowment vectors or in terms of excess demand functions and tax functions.

To keep the presentation simple, let us specify the production technology using an $\mathrm{n} \times \mathrm{k}$ activity analysis matrix $\mathrm{A}$. We again assume that $\mathrm{A}$ includes free disposal activities and does not allow outputs with inputs. Production taxes are specified by an $\mathrm{n} \times \mathrm{k}$ matrix $\mathrm{A}^{*}$ that satisfies $\mathrm{A}^{*} \leq \mathrm{A}$. Here $\mathrm{a}_{\mathrm{ij}}^{*}=\mathrm{a}_{\mathrm{ij}}-\tau_{\mathrm{ij}}\left|\mathrm{a}_{\mathrm{ij}}\right|$ where $\tau_{\mathrm{ij}}$ is the ad valorem tax on the output or input of commodity $i$ in activity $j$. There are no taxes on disposal activities. The revenue from production taxes at prices $p \in R_{+}^{n} \backslash\{0\}$ and activity levels $y \in R_{+}^{k}$ is $p^{\prime}\left(A-A^{*}\right) y$.

An equilibrium of an economy $\left(f, t, A, A^{*}\right)$ is a price vector $\hat{p} \in R_{+}^{n}$, a revenue level $\mathbf{f} \in R_{+}$, and a vector of activity levels $\varphi \in R_{+}^{k}$ such that

- $\hat{p}^{\prime} A^{*} \leq 0$

- $f(\hat{p}, \hat{r})=A y$

- $\hat{\mathrm{f}}=\mathrm{t}(\hat{\mathrm{p}}, \hat{\mathrm{r}})+\hat{\mathrm{p}}^{\prime}\left(\mathrm{A}-\mathrm{A}^{*}\right) \mathrm{y}$

Walras's law implies that $\mathrm{p}^{\prime} \mathrm{A}^{*} \mathrm{y}=0$.

Once again we can use homogeneity to normalize prices to lie in the unit simplex $S$. To bound the set of potential levels of government revenue, we note that the assumption of no outputs without inputs implies that there exists some $\alpha>0$ such that $\|\mathrm{x}\|<\alpha$ for all $\mathrm{x}$ in the production possibility set $\left\{x \in R^{n} \mid x=A y \geq-\sum_{i=1}^{m} w^{i}, y \geq 0\right\}$. Our assumptions on $f$ imply that there exists $\beta>0$ such that $\|\mathrm{f}(\mathrm{p}, \mathrm{r})\| \geq \alpha$ for all $\mathrm{p} \in \mathrm{S}, \mathrm{r} \geq \beta$. Consequently, we can restrict our attention to the nonempty, compact, convex set $S \times[0, \beta]$ in searching for equilibria. To define $g$ : $S \times[0, \beta] \rightarrow S \times[0, \beta]$, we let $g(p, r)=\left(g_{1}(p, r), g_{2}(p, r)\right)$ be the vector $\left(g_{1}, g_{2}\right)$ that solves 


$$
\min (1 / 2)\left[\left(g_{1}-p-f(p, r)\right)^{\prime}\left(g_{1}-p-f(p, r)\right)+\left(g_{2}-t(p, r)\right)^{2}\right]
$$

subject to

$$
\begin{aligned}
& g_{1}^{\prime} A-\left(1+g_{2}-r\right) p^{\prime}\left(A-A^{*}\right) \leq 0 \\
& g_{1}^{\prime} e=1 \\
& 0 \leq g_{2} \leq \beta .
\end{aligned}
$$

Notice that the assumption of free disposal and the first constraint imply that $g_{1} \geq 0$.

Proposition 71 (Kehoe 1985a) $(\hat{p}, \hat{f}, \hat{y})$ is an equilibrium if and only if $(\hat{p}, \hat{\mathrm{f}})$ is a fixed point of $\mathrm{g}$, $(\hat{\rho}, \hat{\imath})=g(\hat{p}, \hat{\mathbf{l}})$.

Proof. The Kuhn-Tucker theorem implies that $\left(g_{1}(p, r), g_{2}(p, r)\right)=g(p, r)$ solves the minimization problem that defines it if and only if there exist Lagrange multipliers $y \in \mathrm{R}_{+}^{\mathrm{k}}, \lambda \in \mathrm{R}$, and $\mu$, $\nu \in \mathrm{R}_{+}$such that

$$
\begin{aligned}
& \mathrm{g}_{1}(\mathrm{p}, \mathrm{r})-\mathrm{p}-\mathrm{f}(\mathrm{p}, \mathrm{r})+\mathrm{Ay}+\lambda \epsilon=0 \\
& \mathrm{~g}_{2}(\mathrm{p}, \mathrm{r})-\mathrm{t}(\mathrm{p}, \mathrm{r})-\mathrm{p}^{\prime}\left(\mathrm{A}-\mathrm{A}^{*}\right) \mathrm{y}+\mu-\nu=0 \\
& {\left[\mathrm{~g}_{1}(\mathrm{p}, \mathrm{r})^{\prime} \mathrm{A}-\left(1+\mathrm{g}_{2}(\mathrm{p}, \mathrm{r})-\mathrm{r}\right) \mathrm{p}^{\prime}\left(\mathrm{A}-\mathrm{A}^{*}\right) \mathrm{y}=0\right.} \\
& \mu \mathrm{g}_{2}(\mathrm{p}, \mathrm{r})=0 \\
& \nu\left(\beta-\mathrm{g}_{2}(\mathrm{p}, \mathrm{r})\right)=0 .
\end{aligned}
$$

Suppose that $(\hat{\rho}, \hat{f})=g(\hat{p}, \hat{f})$ and, for the moment, that $\hat{\mu}=\hat{\nu}=0$. This implies that $\hat{p}^{\prime} A^{*} \leq 0$ and $\hat{t}=t(\hat{P}, \hat{P})+\hat{p}^{\prime}\left(A-A^{*}\right) \hat{y}$. To obtain the remaining equilibrium condition, we multiply the first condition above by $\hat{\mathrm{p}}^{\prime}$ to produce

$$
-\hat{p}^{\prime} f(\hat{p}, \hat{r})+\hat{p}^{\prime} A \hat{y}+\hat{\lambda}=0 .
$$

Walras's law now implies that $\hat{\lambda}=0$, and the first condition becomes $-f(\hat{p}, \hat{\mathrm{r}})+A \hat{y}=0$. That $\mathfrak{t}(\hat{p}, \hat{P}) \geq 0$ and $\hat{p}^{\prime}\left(A+A^{*}\right) \hat{y} \geq 0$ justifies us in ignoring the possibility that $\hat{\mu}>0$. Suppose, 
however, that $\hat{\nu}>0$ at a fixed point $(\hat{p}, \beta)$. Our above reasoning then implies that $-f(\hat{p}, \beta)+$ Ay $>0$, which would contradict our choice of $\beta$. Consequently, any fixed point is an equilibrium. To demonstrate the converse, that any equilibrium is a fixed point, we set $y=9$ and $\lambda=$ $\mu=\nu=0$ in the Kuhn-Tucker conditions.

Kehoe (1985a) develops regularity analysis and an index theorem for economies with taxes and government spending. The index of an equilibrium $(\hat{p}, \mathrm{f}, \mathrm{y})$ is

$$
\operatorname{sgn}\left[\operatorname{det}\left[\begin{array}{cc}
-D_{1} f(\hat{p}, \hat{r}) & B \\
-B^{* \prime} & 0
\end{array}\right]\right] \text {. }
$$

Here $B$ and $B^{*}$ are the matrices whose columns are the activities of $A$ and $A^{*}$, respectively, that are associated with strictly positive activity levels $\hat{\vartheta}_{j}$. A regular economy has this expression nonzero at all equilibria. Once again, $\Sigma$ index $(p, r)=+1$ where the sum is over all equilibria of a regular economy.

Unfortunately, not even the assumptions of a representative consumer or a nonsubstitution theorem technology and endowment pattern, each of which guarantees uniqueness of equilibrium in an economy without taxes, rules out multiplicity of equilibria. The following example illustrates this point. It is based on the analysis of Foster and Sonnenschein (1970); see also Hatta (1977) and Kehoe, Levine, and Romer (1989b).

Example 7.1. Consider an economy with two consumption goods and labor. There is a representative consumer with utility function

$$
u\left(x_{1}, x_{2}, x_{3}\right)=\left\{\begin{array}{cl}
-\left(3-x_{1}\right)^{2}\left(x_{2}+2\right)^{-1} & \text { if } x_{1} \leq 3 \\
x_{1}-3 & \text { if } x_{1} \geq 3
\end{array}\right.
$$


and endowment $\left(\mathrm{w}_{1}, \mathrm{w}_{2}, \mathrm{w}_{3}\right)=(0,0,2)$. There is an ad valorem tax $\tau_{1}=2$ on the consumption of the first good. All tax revenues are returned to the consumer in the form of a lump sum rebate, $r$. The excess demand functions are

$$
\begin{aligned}
& f_{1}(p, r)=\left\{\begin{array}{cl}
0 & \text { if } 9 p_{1}-4 p_{2}-2 p_{3}-r-r \geq 2 p_{3}+r \\
\left(-9 p_{1}+4 p_{2}+4 p_{3}+2 r\right) / 3 p_{1} & \text { if } 2 p_{3}+r \geq 9 p_{1}-4 p_{2}-2 p_{3}-r \geq 0 \\
\left(2 p_{3}+r\right) / 3 p_{1} & \text { if } 0 \geq 9 p_{1}-4 p_{2}-2 p_{3}-r
\end{array}\right. \\
& f_{2}(p, r)=\left\{\begin{array}{cl}
\left(2 p_{3}+r\right) / p_{2} & \text { if } 9 p_{1}-4 p_{2}-2 p_{3}-r \geq 2 p_{3}+r \\
\left(9 p_{1}-4 p_{2}-2 p_{3}-r\right) / p_{2} & \text { if } 2 p_{3}+r \geq 9 p_{1}-4 p_{2}-2 p_{3}-r \geq 0 \\
0 & \text { if } 0 \geq 9 p_{1}-4 p_{2}-2 p_{3}-r
\end{array}\right. \\
& f_{3}(p, r)=-2 .
\end{aligned}
$$

The tax function is $t(p, r)=2 p_{1} f_{1}(p, r)$.

The production technology is specified by the matrix

$$
A=A^{*}=\left[\begin{array}{rrrrr}
-1 & 0 & 0 & 2 & -1 \\
0 & -1 & 0 & -1 & 2 \\
0 & 0 & -1 & -1 & -1
\end{array}\right]
$$


There are three equilibria:

\begin{tabular}{||cccc||}
\hline & Equilibrium 1 & Equilibrium 2 & Equilibrium 3 \\
\hline$\hat{\mathrm{p}}_{1}$ & $1 / 3$ & $1 / 3$ & $1 / 3$ \\
$\mathrm{p}_{2}$ & $1 / 3$ & $1 / 3$ & $1 / 3$ \\
$\hat{\mathrm{P}}_{3}$ & $1 / 3$ & $1 / 3$ & $1 / 3$ \\
$\hat{\mathrm{f}}$ & $4 / 3$ & $2 / 3$ & 0 \\
$\hat{\mathrm{y}}_{4}$ & $4 / 3$ & 1 & $2 / 3$ \\
$\hat{\mathrm{y}}_{5}$ & $2 / 3$ & 1 & $4 / 3$ \\
$\mathrm{f}_{1}$ & 2 & 1 & 0 \\
$\mathrm{f}_{2}$ & 0 & 1 & 2 \\
$\hat{\mathrm{Q}}$ & $-1 / 2$ & $-4 / 3$ & $-9 / 4$ \\
\hline
\end{tabular}

Equilibria 1 and 3 have index +1 , and equilibrium 2 has index -1 .

\subsection{Dynamic Economies with Taxes and Externalities}

The existence of equilibrium in economies whose equilibria are not necessarily Pareto efficient can be established using sequences of truncations as in Section 6. Similar computational techniques can be used to approximate equilibria. Unfortunately, dynamic economies with taxes and externalities, like overlapping generations economies, can have robust continua of equilibria.

Example 7.2. Consider a dynamic economy with a representative consumer and a one-sector production technology. The consumer derives utility not only from consumption but also from investment. The consumer purchases the investment good, perhaps human capital in the form of education that is valued for its own sake, in one period and sells it to the representative firm in the next. He faces an ad valorem tax on purchases of the consumption good. All tax revenues are rebated in lump-sum form to the consumer. He chooses $\left(c_{1}, x_{1}\right),\left(c_{2}, x_{2}\right), \ldots$, to solve 


$$
\max \sum_{t=1}^{\infty} \gamma^{t-1} u\left(c_{t}, x_{t}\right)
$$

subject to

$$
\begin{aligned}
& \sum_{t=1}^{\infty}\left(p_{t}(1+\tau) c_{t}+p_{t} x_{t}\right) \leq \sum_{t=1}^{\infty}\left(y_{t}+r_{t} k_{t}\right) \\
& k_{1} \leq \bar{k}_{1} \\
& k_{t} \leq x_{t-1} \\
& c_{t}, \quad x_{t} \geq 0 .
\end{aligned}
$$

Here $x_{t}$ is the amount of the investment good purchased by the consumer in period $t, p_{t}$ is the price paid, $r_{t+1}$ is the price paid by the firm for the same good in period $t+1$, and $y_{t}$ is the consumer's labor income plus tax rebate

$$
y_{t}=\left(p_{t} f\left(k_{t}\right)-r_{t} k_{t}\right)+\tau p_{t} k_{t}
$$

The representative firm chooses $k_{1}, k_{2}$ to solve

$$
\max \sum_{t=1}^{\infty}\left(p_{t} f\left(k_{t}\right)-r_{t} k_{t}\right)
$$

In equilibrium, consumers maximize utility taking $p_{t}, y_{t}$, and $r_{t}$ as given, producers maximize profits taking $p_{t}$ and $r_{t}$ as given, $k_{t}=x_{t-1}$, and

$$
c_{t}+k_{t+1} \leq f\left(k_{t}\right), \quad t=1,2, \ldots
$$

The first-order conditions for the consumer's problem are

$$
\begin{aligned}
& \gamma^{t-1} u_{1}\left(c_{t}, k_{t+1}\right)-\lambda p_{t}(1+\tau)=0 \\
& \gamma^{t-1} u_{2}\left(c_{t}, k_{t+1}\right)-\lambda\left(p_{t}-r_{t+1}\right)=0, \quad t=1,2, \ldots
\end{aligned}
$$


The first-order condition for the firm's problem is

$$
\mathrm{p}_{\mathrm{t}} \mathrm{f}^{\prime}\left(\mathbf{k}_{\mathrm{t}}\right)-\mathrm{r}_{\mathrm{t}}=0, \quad \mathrm{t}=1,2, \ldots
$$

Combining these three conditions to eliminate $p_{t}$ and $r_{t}$, we obtain

$$
(1+\tau) u_{2}\left(c_{t}, k_{t+1}\right)-u_{1}\left(c_{t}, k_{t+1}\right)+\gamma u_{1}\left(c_{t+1}, k_{t+2}\right) f^{\prime}\left(k_{t+2}\right)=0, \quad t=1,2, \ldots
$$

Substituting in the feasibility condition

$$
c_{t}=f\left(k_{t}\right)-k_{t+1}
$$

yields a second-order difference equation in $k_{t}$.

This difference equation requires two initial conditions. The value of $k_{1}$ is given. To find how much freedom there is in choosing $k_{2}$, we linearize the difference equation around a stationary solution of $\hat{k}$. We obtain

$$
\alpha_{2}\left(k_{t+2}-\hat{k}\right)+\alpha\left(k_{t+1}-\hat{k}\right)+\alpha_{0}\left(k_{t}-\hat{k}\right)=0
$$

where

$$
\begin{aligned}
& \alpha_{0}=\left((1+\tau) \mathrm{u}_{21}-\mathrm{u}_{11}\right) \mathrm{f}^{\prime} \\
& \alpha_{1}=-(1+\tau) \mathrm{u}_{21}+(1+\tau) \mathrm{u}_{22}+\mathrm{u}_{11}-\mathrm{u}_{12}+\gamma \mathrm{u}_{11} \mathrm{f}^{2}+\gamma \mathrm{u}_{1} \mathrm{f}^{\prime \prime} \\
& \alpha_{2}=\gamma\left(\mathrm{u}_{12}-\mathrm{u}_{11}\right) \mathrm{f}^{\prime}
\end{aligned}
$$

Consider the example where $\gamma=3 / 4, \tau=2$,

$$
\begin{aligned}
& \mathrm{u}(\mathrm{c}, \mathrm{k})=8 \mathrm{c}+(157 / 48) \mathrm{k}-(1 / 2)\left(5 \mathrm{c}^{2}+4 \mathrm{ck}+\mathrm{k}^{2}\right) \\
& \mathrm{f}(\mathrm{k})=\left(55+10 \mathrm{k}-\mathrm{k}^{2}\right) / 32
\end{aligned}
$$

In this case $t=\hat{k}=1$ is a stationary solution where $u_{11}=-5, u_{12}=u_{21}=-2, u_{22}=-1$, $u_{1}=1, f^{\prime}=1 / 4$, and $f^{\prime \prime}=-1 / 16$. This implies that $\alpha_{0}=-1 / 4, \alpha_{1}=-9 / 32$, and $\alpha_{2}=9 / 16$. The two roots of the characteristic equation

$$
\alpha_{2} \lambda^{2}+\alpha_{1} \lambda+\alpha_{0}=0
$$


are $\lambda=0.2500 \pm 0.7120 \mathrm{i}$. Since both roots have moduli less than one, there is a continuum of equilibria for which $k_{t}$ converges to $\bar{k}=1$.

Example 7.3. Consider another dynamic economy with a representative consumer and a one-sector production technology. The consumer chooses $c_{1}, c_{2}, \ldots$ to solve

$$
\max \sum_{\mathfrak{t}=1}^{\infty} \gamma^{\mathfrak{t}-1} \mathrm{u}\left(\mathrm{c}_{\mathfrak{t}}\right)
$$

subject to

$$
\begin{aligned}
& \sum_{\mathfrak{t}=1}^{\infty} p_{\mathfrak{t}} c_{\mathfrak{t}} \leq \sum_{\mathfrak{t}=1}^{\infty} y_{t} \\
& c_{\mathfrak{t}} \geq 0 .
\end{aligned}
$$

Here $y_{t}$ is the total of labor and capital income in period $t$. There is a congestion externality in that output depends not only on the inputs of capital $k_{t}$ and the fixed amount of labor but on the average amount capital $\mathrm{K}_{\mathrm{t}}$. The representative firm chooses $\mathrm{k}_{1}, \mathrm{k}_{2}, \ldots$ to solve

$$
\max \sum_{t=1}^{\infty}\left(p_{t} f\left(k_{t}, K_{t}\right)-p_{t} k_{t+1}\right)-r_{1} k_{1}
$$

Here the firm cannot control the average $K_{t}$ although, in equilibrium $K_{t}=k_{t}$. The other equilibrium conditions are

$$
\begin{aligned}
& y_{t}=p_{t} f\left(k_{t}, K_{t}\right) \\
& c_{t}+k_{t+1} \leq f\left(k_{t}, K_{t}\right) \\
& k_{1} \leq \bar{k}_{1} .
\end{aligned}
$$

The first-order conditions for the consumer's problem

$$
\gamma^{t-1} u^{\prime}\left(c_{t}\right)-\lambda p_{t}=0
$$


can be combined with the first-order conditions for the firm's problem

$$
p_{t+1} f_{1}\left(k_{t+1}, K_{t+1}\right)-p_{t}=0
$$

to yield

$$
\gamma u^{\prime}\left(c_{t+1}\right) f_{1}\left(k_{t+1}, K_{t+1}\right)-u^{\prime}\left(c_{t}\right)=0 .
$$

Substituting in the equilibrium conditions, we obtain a second-order difference equation in $k_{t}$

$$
\gamma u^{\prime}\left(f\left(k_{t+1}, k_{t+1}\right)-k_{t+2}\right) f_{1}\left(k_{t+1}, k_{t+1}\right)-u^{\prime}\left(f\left(k_{t}, k_{t}\right)-k_{t+1}\right)=0 .
$$

Once again, we have only one initial condition $\mathrm{k}_{1}$. Linearizing around a stationary solution $\overline{\mathrm{k}}$, we obtain

where

$$
\alpha_{2}\left(k_{t+2}-\bar{k}\right)+\alpha_{1}\left(k_{t+1}-\bar{k}\right)+\alpha_{0}\left(k_{t}-\bar{k}\right)=0
$$

$$
\begin{aligned}
& \alpha_{0}=-u^{\prime \prime}\left(f_{1}+f_{2}\right) \\
& \alpha_{1}=\gamma u^{\prime \prime} f_{1}\left(f_{1}+f_{2}\right)+\gamma u^{\prime}\left(f_{11}+f_{12}\right)+u^{\prime \prime} \\
& \alpha_{2}=-\gamma u^{\prime \prime} f_{1} .
\end{aligned}
$$

In the case where $\bar{c}=\bar{k}=1, \gamma=3 / 4, u^{\prime}=2, u^{\prime \prime}=-1, f_{1}=4 / 3, f_{2}=-1, f_{11}=-1$, and $\mathrm{f}_{12}=2$, we can calculate $\alpha_{0}=-1 / 3, \alpha_{1}=1 / 6$, and $\alpha_{2}=1$. The two roots of the characteristic equation are $1 / 2,-2 / 3$. Since both roots have moduli less than one, there is a continuum of equilibria for which $k_{t}$ converges to $\bar{k}=1$.

As in Example 7.2, we can choose $\mathrm{u}$ and $\mathrm{f}$ as quadratic functions so that the linear approximation to the equilibrium conditions is exact.

Bemark. Similar examples of multiplicity of equilibria in economies with externalities have been constructed by Howitt and McAfee (1988), Spear (1988), and Kehoe, Levine, and Romer (1989a). 


\subsection{Characterizing Equilibria as Solutions to Optimization Problems}

As we have mentioned, the computational methods for overlapping generations economies can also be applied to economies with taxes and externalities. An alternative is to characterize equilibria as solutions to optimization problems where some of the parameters of the optimization problem are endogenously determined. This characterization is formally similar to the Negishi approach described in Section 3, where the welfare weights $\alpha$ are endogenously determined, although there is now no interpretation in terms of Pareto efficiency.

Example 7.4 (7.1 Revisited). Consider the "Pareto" problem

$$
\max u\left(x_{1}, x_{2}, x_{3}\right)-z x_{1}
$$

subject to

$$
\begin{aligned}
& x-w \leq A y \\
& x, \quad y \geq 0
\end{aligned}
$$

where the function $u$, the vector $w$, and the matrix $A$ are as in Example 7.1. Letting $p \in R_{+}^{3}$ be the Langrange multipliers, we can write the first order conditions as

$$
\begin{aligned}
& \mathrm{u}_{1}\left(\mathrm{x}_{1}, \mathrm{x}_{2}, \mathrm{x}_{3}\right)-\mathrm{x}-\mathrm{p}_{1} \leq 0,=0 \text { if } \mathrm{x}_{1}>0 \\
& \mathrm{u}_{2}\left(\mathrm{x}_{1}, \mathrm{x}_{2}, \mathrm{x}_{3}\right)-\mathrm{p}_{2} \leq 0,=0 \text { if } \mathrm{x}_{2}>0 \\
& \mathrm{p}^{\prime} \mathrm{A} \leq 0, \mathrm{p}^{\prime} \mathrm{Ay}=0 \\
& \text { Ay }-\mathrm{x}+\mathrm{w}=0 .
\end{aligned}
$$

These are the same as the equilibrium conditions of Example 7.1 in the case where $z=\tau_{1}$. Equilibria are, therefore, fixed points of $g(z)=\tau p_{1}(z)$.

For a fixed value of $z$, we can solve the maximization problem and find the solution $(x(z), y(z), p(z))$. For any value of $z$ there is a unique solution. 


\begin{tabular}{||cclc|}
\hline & $z \geq 15 / 16$ & $15 / 16 \geq z \geq 3 / 4$ & $3 / 4 \geq z$ \\
\hline$x_{1}$ & 0 & $4-(1-z)^{-1 / 2}$ & 2 \\
$x_{2}$ & 2 & $(1-z)^{-1 / 2}-2$ & 0 \\
$y_{4}$ & $2 / 3$ & $2-(1-z)^{-1 / 2} / 3$ & $4 / 3$ \\
$y_{5}$ & $4 / 3$ & $(1-z)^{-1 / 2} / 3$ & $2 / 3$ \\
$p_{1}$ & $9 / 16$ & $2-z-2(1-z)^{1 / 2}$ & $1-z$ \\
$p_{2}$ & $9 / 16$ & $2-z-2(1-z)^{1 / 2}$ & $1-z$ \\
$p_{3}$ & $9 / 16$ & $2-z-2(1-z)^{1 / 2}$ & $1-z$ \\
\hline
\end{tabular}

The function $g(z)=2 p_{1}(z)$ has three fixed points, $z^{1}=2 / 3, z^{2}=8 / 9$, and $z^{3}=9 / 8$, which correspond to the three equilibria of Example 7.1.

The equilibria of the dynamic economies in Examples 7.2 and 7.3 can also be characterized as solutions to optimization problems with endogenous parameters. In each case, however, there is an infinite number of endogenous parameters. In the case of the dynamic economy with externalities, for example, the "Pareto" problem is to choose $\left(c_{1}, k_{1}\right),\left(c_{2}, k_{2}\right), \ldots$ to solve

$$
\max \sum_{\mathbf{t}=1}^{\infty} \gamma^{\mathbf{t}-1} \mathbf{u}\left(c_{\mathfrak{t}}\right)
$$

subject to

$$
\begin{aligned}
& c_{t}+k_{t} \leq f\left(k_{t}, x_{t}\right) \\
& k_{1} \leq \bar{k}_{1} \\
& c_{t}, \quad k_{t} \geq 0 .
\end{aligned}
$$

For the solution to this problem to be an equilibrium, the parameters $z_{1}, z_{2}, \ldots$ must solve the fixed point problem $z_{t}=k_{t}\left(x_{t}\right), t=1,2, \ldots$ (See Kehoe, Levine, and Romer 1989a for details and references.) 
Sometimes the equilibrium of an economy with taxes or externalities solves an optimization problem without additional equilibrium conditions. Becker (1985), for example, considers a model in which the representative consumer solves

$$
\max \sum_{t=1}^{\infty} \gamma^{t-1} u\left(c_{t}\right)
$$

subject to

$$
\begin{aligned}
& \sum_{t=1}^{\infty} p_{t}\left(c_{t}+k_{t+1}\right) \leq \sum_{t=1}^{\infty}\left(y_{t}+(1-\tau) r_{t} k_{t}\right) \\
& k_{1} \leq \vec{k}_{1} \\
& c_{t}, \quad k_{t} \geq 0 .
\end{aligned}
$$

Here $y_{t}=p_{t} f\left(k_{t}\right)-r_{t} k_{t}+\pi_{t} k_{t}$ is the consumer's labor income plus a lump sum rebate. Becker shows that an equilibrium also solves

$$
\max \sum_{t=0}^{\infty}(\gamma(1-\tau))^{t} u\left(c_{t}\right)
$$

subject to

$$
\begin{aligned}
& c_{t}+k_{t+1} \leq f\left(k_{t}\right), \quad t=1,2, \ldots \\
& k_{1} \leq \bar{k}_{1} \\
& c_{t}, \quad k_{t} \geq 0 .
\end{aligned}
$$

Danthine and Donaldson (1986) extend Becker's analysis to economies that allow uncertainty. Judd (1987) shows that some similar, continuous time tax models also have equilibria that solve optimization problems without side conditions. He further argues that, although there are few cases in which equilibria of tax models can be computed exactly by solving an optimization problem 
without additional conditions on some of the parameters, research in this area may be helpful in updating guesses in iterative methods for computing equilibria.

Suppose, for example, that we characterize the equilibria of either the dynamic economy with taxes in Example 7.2 or the dynamic economy with externalities in Example 7.3 as solutions to optimization problems that depend on a sequence $z_{1}, z_{2}, \ldots$ of endogenous parameters. We start with a guess for this sequence, say, the steady state. We then solve the optimization problem for this guess of $z_{t}$ and then use the solution to update $z_{t}$ and so on. Kydland and Prescott (1977) and Whiteman (1983) discuss algorithms of this sort for computing equilibria in economies with externalities; Braun (1988), Chang (1988), and McGrattan (1988) have applied such algorithms to economies with taxes; and Ginsburgh and van der Heyden (1988) have applied such an algorithm to an economy with institutionally fixed prices. Further research is needed to see whether this algorithm has any advantages over alternatives. 


\section{References}

Acton, F. S. (1970) Numerical methods that work. New York: Harper and Row.

Antonelli, G. B. (1886) Sulla teoria matematica della economic politica. Pisa: Tipografia del Folchetto; translated as Antonelli, G. B. (1971) 'On the mathematical theory of political economy,' in J. S. Chipman, L. Hurwicz, M. K. Richter, and H. F. Sonnenschein, eds., Preferences, utility and demand: A Minnesota symposium. New York: Harcourt Brace Jovanovich.

Araujo, A. (1989) 'On the differentiability of the policy function,' unpublished manuscript, Instituto de Matematica Pura e Aplicada, Rio de Janeiro.

Araujo, A. and J. A. Scheinkman (1977) 'Smoothness, comparative dynamics, and the turnpike property,' Econometrica, 45: 601-620.

Arrow, K. J. (1951) 'An extension of the basic theorems of classical welfare economics,' in J. Neyman, ed., Proceedings of the second Berkeley symposium on mathematical statistics and probability. Berkeley: University of California Press.

Arrow, K. J. (1953) 'Le rôle des valeurs boursiéres pour la répartition la meilleure des risques,' Econometrie. Paris: Centre National de la Recherche Scientifique, 41-48.

Arrow, K. J., H. D. Block, and L. Hurwicz (1959) 'On the stability of the competitive equilibrium II,' Econometrica, 27: 82-109.

Arrow, K. J. and F. H. Hahn (1971) General competitive analysis. San Francisco: Holden-Day. Arrow, K. J. and L. Hurwicz (1958) 'On the stability of competitive equilibrium 1,' Econometrica, 26: $522-552$.

Auerbach, A. J. and L. J. Kotlikoff (1987) Dynamic fiscal analysis. Cambridge: Cambridge University Press. 
Balasko, Y., D. Cass, and K. Shell (1980) 'Existence of competitive equilibrium in a general overlapping generations model,' Journal of Economic Theory, 23: 307-322.

Becker, R. A. (1985) 'Capital income taxation and perfect foresight,' Journal of Public Economics, 26: $147-167$.

Bellman, R. (1957) Dynamic programming. Princeton, NJ: Princeton University Press.

Benhabib, J. and R. H. Day (1982) 'A characterization of erratic dynamics in the overlapping generations model,' Journal of Economic Dynamics and Control, 4: 37-55.

Benhabib, J. and S. Nishimura (1979) 'The Hopf bifurcation and the structure and stability of closed orbits with heterogeneous steady states in multi-sector models of optimal growth,' Journal of Economic Theory, 21: 421-444.

Benveniste, L. M. and J. A. Scheinkman (1979) 'On the differentiability of the value function in dynamic models of economics,' Econometrica, 47: 727-732.

Bertsekas, D. (1976) Dynamic programming and stochastic control. New York: Academic Press.

Bewley, T. F. (1980) 'The permanent income hypothesis and long-run economic stability,' Journal of Economic Theory, 22: 377-394.

Bewley, T. F. (1982) 'An integration of equilibrium theory and turnpike theory,' Journal of Mathematical Economics, 10: 233-267.

BlackwelI, D. (1965) 'Discounted dynamic programming,' Annals of Mathematical Statistics, 36: $226-235$.

Blume, L., D. Easley, and M. O'Hara (1982) 'Characterization of optimal plans for stochastic dynamic programs,' Journal of Economic Theory, 28: 221-234.

Boldrin, M. and L. Montrucchio (1986) 'On the indeterminacy of capital accumulation paths,' Journal of Economic Theory, 40: 26-39. 
Boldrin, M. and L. Montrucchio (1988) 'On the differentiability of policy functions,' unpublished manuscript, University of California-Los Angeles.

Braun, R. A. (1988) 'The dynamic interaction of distortionary taxes and aggregate variables in postwar U.S. data,' Unpublished manuscript, Carnegie-Mellon University.

Brock, W. A. and L. Mirman (1972) 'Optimal economic growth and uncertainty: the discounted case,' Journal of Economic Theory, 4: 479-513.

Brown, D. J. (1989) 'Equilibrium analysis with nonconvex technologies,' in W. Hildenbrand and H. Sonnenschein, eds., Handbook of Mathematical Economics, vol. IV. Amsterdam: North-Holland, forthcoming.

Burke, J. L. (1986) 'On the existence of equilibria in dynamic production economies,' unpublished manuscript, Texas A\&M University.

Burke, J. L. (1988) 'On the existence of price equilibria in dynamic economies,' Journal of Economic Theory, 44: 281-300.

Burmeister, E. (1980) Capital theory and dynamics. Cambridge: Cambridge University Press.

Chang, L. J. (1988) 'Corporate taxes, disaggregated capital markets, and business cycles', unpublished manuscript, Carnegie-Mellon University.

Chipman, J. S. (1974) 'Homothetic preferences and aggregation,' Journal of Economic Theory, 8: 26-38.

Christiano, L. J. (1990) 'Solving the stochastic growth model by linear-quadratic approximation and by value-function iteration,' Journal of Business and Economic Statistics, 8: 23-26.

Coles, J. L. (1985) 'Equilibrium turnpike theory with constant returns to scale and possible heterogeneous discount factors,' International Economic Review, 26: 671-679.

Dana, R. A. and C. Le Van (1987) 'Optimal growth and Pareto-optimality,' Unpublished manuscript, C.E.P.R.E.M.A.P. 
Danthine, J. P. and J. B. Donaldson (1986) 'A note on the effects of capital income taxation on the dynamics of a competitive economy, Journal of Public Economics, 28: 255-265.

Debreu, G. (1954) 'Valuation equilibrium and Pareto optimum,' Proceedings of the National Academy of Sciences, 40: 588-592.

Debreu, G. (1959) Theory of value. New York: Wiley.

Debreu, G. (1970) 'Economies with a finite set of equilibria,' Econometrica, 38: 387-392.

Debreu, G. (1972) 'Smooth preferences,' Econometrica, 40: 603-612.

Debreu, G. (1974) 'Excess demand functions,' Journal of Mathematical Economics, 1: 15-23.

Dierker, E. (1972) 'Two remarks on the number of equilibria of an economy,' Econometrica, 40: $951-953$.

Dierker, E. (1974) Topological methods in Walrasian economics. Lecture Notes on Economics and Mathematical Sciences, 92. Berlin: Springer-Verlag.

Dierker, E. (1982) 'Regular economies,' in K. J. Arrow and M. P. Intriligator, eds., Handbook of mathematical economics, vol. II. Amsterdam: North-Holland.

Dierker, E., and H. Dierker (1972) 'The local uniqueness of equilibria,' Econometrica, 40: $867-881$.

Diewert, W. E. (1982) 'Duality approaches to microeconomic theory,' in K. J. Arrow and M. Intriligator, eds., Handbook of mathematical economics, vol. II. Amsterdam: North-Holland.

Drexler, F. J. (1978) 'A homotopy method for the calculation of all zeros of zero-dimensional polynomial ideals,' in H. Wacker, ed., Continuation Methods. New York: Academic Press.

Duffie, D., J. Geanakoplos, A. Mas-Colell, and A. McLennan (1988) 'Stationary Markov equilibria,' unpublished manuscript, University of Minnesota. 
Eaves, B. C. (1971) 'On the basic theorem of complementarity,' Mathematical Programming, I: $68-75$

Eaves, B. C. (1972) 'Homotopies for computation of fixed points,' Mathematical Programming, 3: $1-22$

Eaves, B. C. (1976) 'A short course in solving equations with PL homotopies,' in R. W. Cottle and C. E. Lemke, eds,, 'Nonlinear programming,' SIAM-AMS Proceedings, 9: 73-143.

Eaves, B. C. and H. E. Scarf (1976) 'The solution of systems of piecewise linear equations,' Mathematics of Operations Research, 1: 1-27.

Eisenberg, B. (1961) 'Aggregation of utility functions,' Management Science, 7: 337-350.

Ekeland, I. and J. A. Scheinkman (1986) 'Transversality conditions for some infinite horizon discrete time optimization problems,' Mathematics of Operations Research, 11: 216-229. van den Elzen, A., G. van der Laan, and D. Talman (1989) 'An adjustment process for an exchange economy with linear production technologies,' unpublished manuscript, Tilburg University.

Epstein, L. G. and S. E. Zin (1989) 'Substitution, risk aversion, and the temporal behavior of consumption and the asset returns: A theoretical framework,' Econometrica, 57: 937-969.

Foster, E. and H. Sonnenschein (1970) 'Price distortions and economic welfare' Econometrica, 38: 281-297.

Freixas, X. and A. Mas-Colell (1987) 'Engel curves leading to the weak axiom in the aggregate,' Econometrica, 55: 515-531.

Gale, D. (1960) The theory of linear economic models. New York: McGraw-Hill.

Gale, D. (1973) 'Pure exchange equilibrium of dynamic economic models,' Journal of Economic Theory, 6: 12-36.

Garcia, C. B. and W. I. Zangwill (1979) 'Determining all solutions to certain systems of nonlinear equations,' Mathematics of Operations Research 4, 1-14. 
Garcia, C. B. and W. I. Zangwill (1981) Pathways to solutions, fixed points, and equilibria. Englewood Cliffs, NJ: Prentice-Hall.

Geanakoplos, J. (1984) 'Utility functions for Debreu's excess demand,' Journal of Mathematical Economics, 13: 1-9.

Geanakoplos, J. D. and D. J. Brown (1985) 'Comparative statics and local determinacy in OLG economies: An application of the multiplicative ergotic theorem,' Cowles Foundation Discussion Paper 773.

Gear, C. W. (1971) Numerical initial value problems in ordinary differential equations. Englewood Cliffs, N. J.: Prentice-Hall.

Ginsburgh, V. A. and L. van der Heyden (1988) 'On extending the Negishi approach to computing equilibria: the case of government price support policies," Journal of Economic Theory, 44: 168-178.

Gorman, W. M. (1953) 'Community preference fields,' Econometrica, 21: 63-80.

Grandmont, J. M. (1985) 'On endogenous competitive business cycles,' Econometrica, 53: 995-1045.

den Haan, W. J. and A. Marcet (1990) 'Solving the stochastic growth model by parameterizing expectations,' Journal of Business and Economic Statistics, 8: 31-34.

Hahn, F. H. (1958) 'Gross substitutability and the dynamic stability of general equilibrium,' Econometrica, 26: 169-170.

Hahn, F. H. (1982) 'Stability,' in K.J. Arrow and M.D. Intriligator, eds., Handbook of mathematical economics, vol. II. Amsterdam: North-Holland.

Hansen, G. D. and E. C. Prescott (1991) 'Recursive methods for computing equilibria of business cycle models,' Discussion Paper 36, Institute for Empirical Macroeconomics.

Harris, M. (1987) Dynamic economic analysis. New York: Oxford University Press. 
Hatta, T. (1977) 'A theory of piecemeal policy recommendations,' Review of Economic Studies, 64: $1-21$.

Helpman, E. (1976) 'Solutions of general equilibrium problems for a trading world,' Econometrica, 44: 547-559.

Henry, C. (1972) 'Differential equations with discontinuous right-hand side for planning procedures,' Journal of Economic Theory, 4: 545-551.

Henry, C. (1973) 'An existence theorem for a class of differential equations with multivalued right-hand side,' Journal of Mathematical Analysis and Applications, 41: 179-186.

Hildenbrand, W. (1983) 'On the "law of demand",' Econometrica, 51: 997-1019.

Hildenbrand, W. (1989), 'The weak axiom of revealed preference is strong,' Econometrica, 57: 979-987.

Hirsch, M. W. (1963) 'A proof of the nonretractability of a cell into its boundary,' Proceedings of the American Mathematical Society, 14: 364-365.

Hirsch, M. W. and S. Smale (1974) Differential equations, dynamical systems, and linear algebra. New York: Academic Press.

Howitt, P. and R. P. MacAfee (1988) 'Stability of equilibria with externalities,' Quarterly Journal of Economics, 103: 261-277.

Irwin, M. C. (1980) Smooth dynamical systems. New York: Academic Press.

Jacobs, D. A. H., ed. (1977) The state of the art in numerical analysis. New York: Academic Press.

Jerison M. (1984) 'Aggregation and pairwise aggregation of demand when the distribution of income is fixed,' Journal of Economic Theory, 33: 1-31.

John, R. (1989) 'The two sector CES model,' unpublished manuscript, University of Bonn. 
Jones, L. E. and R. E. Manuelli (1990) 'A convex model of equilibrium growth,' unpublished manuscript, Northwestern University.

Judd, K. L. (1987) 'Useful planning equivalents of taxed economies,' unpublished manuscript, Hoover Institution.

Judd, K. L. (1991) 'Minimum weighted residual methods for solving aggregate growth models,' Discussion Paper 49, Institute for Empirical Macroeconomics.

Keenan, D. (1981) 'Further remarks on the global Newton method,' Journal of Mathematical Economics, 8: 159-165.

Kehoe, T. J. (1980) 'An index theorem for general equilibrium models with production," Econometrica, 48: 1211-1232.

Kehoe, T. J. (1982) 'Regular production economies,' Journal of Mathematical Economics, 10: 147-176.

Kehoe, T. J. (1983) 'Regularity and index theory for economies with smooth production technologies,' Econometrica, 51: 895-917.

Kehoe, T. J. (1984) "Computing all of the equilibria of economies with two factors of production, Journal of Mathematical Economics, 13: 207-223.

Kehoe, T. J. (1985a) 'The comparative statics properties of tax models,' Canadian Journal of Economics, 18: 314-334.

Kehoe, T. J. (1985b) 'Multiplicity of equilibria and comparative statics,' Quarterly Journal of Economics, 100: 119-147.

Kehoe, T. J. (1985c) 'A numerical investigation of multiplicity of equilibria,' in A. S. Manne, ed., Economic equilibrium: model formulation and solution. Mathematical Programming Study 23. Amsterdam: North-Holland. 
Kehoe, T. J. (1986) 'Gross substitutability and the weak axiom of revealed preference,' Journal of Mathematical Economics, forthcoming.

Kehoe, T. J. and D. K. Levine (1984) 'Regularity in overlapping generations exchange economies,' Journal of Mathematical Economics, 13: 69-93.

Kehoe, T. J. and D. K. Levine (1985) 'Comparative statics and perfect foresight in infinite horizon economies,' Econometrica, 53: 433-453.

Kehoe, T. J. and D. K. Levine (1990a) 'Computing equilibria of deterministic dynamic economies,' unpublished manuscript, Federal Reserve Bank of Minneapolis.

Kehoe, T. J. and D. K. Levine (1990b) 'The economics of indeterminacy in overlapping generations models,' Joumal of Public Economics, 42: 219-43.

Kehoe, T. J., D. K. Levine, and P. M. Romer (1989a) 'On characterizing the equilibria of economies with externalities and taxes as solutions to optimization problems,' Economic Theory, forthcoming.

Kehoe, T. J., D. K. Levine, and P. M. Romer (1989b) 'Steady states and determinacy in economies with infinitely lived consumers,' in: G. Feiwel, ed. Joan Robinson and modern economic theory. New York: Macmillan.

Kehoe, T. J., D. K. Levine, and P. M. Romer (1990) 'Determinacy of equilibria in dynamic models with finitely many consumers,' Journal of Economic Theory, 50: 1-21.

Kehoe, T. J., D. K. Levine, A. Mas-Colell, and M. Woodford (1991) 'Gross substitutability in large-scale economies,' Journat of Economic Theory, 54: 1-25.

Kehoe, T. J., D. K. Levine, A. Mas-Colell, and W. R. Zame (1989) 'Determinacy of equilibrium in large-square economies,' Journal of Mathematical Economics, 18: 231-262.

Kehoe, T. J. and A. Mas-Colell (1984) 'An observation on gross substitutability and the weak axiom of revealed preference,' Economics Letters, 15: 241-243. 
Kehoe, T. J. and J. Whalley (1985) 'Uniqueness of equilibrium in large scale numerical general equilibrium models,' Journal of Public Economics, 28: 247-254.

Keller, H. B. (1968) Numerical methods of two-point boundary-value problems. Waltham: Blaisdell.

Kellogg, R. B., T. Y. Li, and J. Yorke (1976) 'A constructive proof of the Brouwer fixed-point theorem and computational results,' SIAM Journal of Numerical Analysis, 13: 473-483.

Kihlstrom, R., A. Mas-Colell, and H. Sonnenschein (1976) 'The demand theory of the weak axiom of revealed preference,' Econometrica, 44: 971-978.

Kirkpatrick, S., C. D. Gelatt, and M. P. Vecchi (1983) 'Optimization by simulated annealing, Science, 220: 671-680.

Koopmans, T. C., ed. (1951) Activity analysis of allocation and production. New York: Wiley.

Koopmans, T. C. (1957) Three essays on the state of economic science. New York: McGraw-Hill.

Koopmans, T. C., P. A. Diamond, and R. E. Williamson (1964) 'Stationary utility and time perspective,' Econometrica, 32: 82-100.

Kuhn, H. W. and J. G. MacKinnon (1975) 'Sandwich method for finding fixed points," Journal of Optimization Theory and Applications, 17: 189-204.

Kydland, F. E. and E. C. Prescott (1977) 'Rules rather than discretion: The inconsistency of optimal plans,' Journal of Political Economy, 85: 473-491.

Kydland, F. E. and E. C. Prescott (1980) 'A competitive theory of fluctuations and the feasibility and desirability of stabilization policies,' in S. Fischer, ed., Rational Expectations and Economic Policy. Chicago: University of Chicago Press.

Kydland, F. E. and E. C. Prescott (1982) 'Time to build and aggregate fluctuations,' Econometrica, 50: $1345-1371$.

van der Laan, G. and A. J. J. Talman (1980) 'An improvement of fixed points algorithms by using a good triangulation," Mathematical Programming, 18: 274-285. 
van der Laan, G. and A. J. J. Talman (1987) 'Adjustment processes for finding economic equilibria, in A. J. J. Talman and G. van der Laan, eds., The computation and modelling of economic equilibria. Amsterdam: North-Holland.

van Laarhoven, P. J. M. and E. H. L. Aarts (1988) Simulated annealing: Theory and applications. Boston: D. Reidel Publishing Company.

Laitner, J. (1984) 'Transition time paths for overlapping generations models,' Journal of Economic Dynamics and Control, 7: 111-129.

Laitner, J. (1990) 'Tax changes and phase diagrams for an overlapping generations model,' Journal of Political Economy, 98: 193-220.

Lang, S. (1983) Real analysis. Reading, Mass.: Addison-Wesley.

Lipton, D., J. Poterba, J. Sachs, and L. Summers (1982) 'Multiple shooting in rational expectations models,' Econometrica, 50: 1329-1334.

Long J. B. and C. I. Plosser (1983), 'Real business cycles,' Journal of Political Economy, 91: 39-69.

Lucas, R. E. (1987) Models of business cycles. New York: Blackwell.

Lucas, R. E. (1988) 'On the mechanics of economic development,' Journal of Monetary Economics, 22: $3-42$.

Lucas, R. E. and N. Stokey (1984) 'Optimal growth with many consumers,' Journal of Economic Theory, 32: 139-171.

Luenberger, D. G. (1969) Optimization by vector space methods. New York: Wiley.

McFadden, D., A. Mas-Colell, R. R. Mantel, and M. K. Richter (1974), 'A characterization of community excess demand functions,' Journal of Economic Theory, 10: 265-268.

McGrattan, E. R. (1988) 'The macroeconomic effects of tax policy in an equilibrium model,' unpublished manuscript, Stanford University. 
McKenzie, L. W. (1955) 'Equality of factor prices in world trade,' Econometrica, 23: 239-257.

McKenzie, L. W. (1959) 'On the existence of general equilibrium for a competitive market, Econometrica, 27: 54-71.

McKenzie, L. W. (196l) 'On the existence of general equilibrium for a competitive market: some corrections,' Econometrica, 29: 247-248.

McKenzie, L. W. (1987) 'Turnpike theory,' in J. Eatwell, M. Milgate, and P. Newman, eds., The New Palgrave: A Dictionary of Economics. New York: Stockton Press.

Mantel, R. R. (1971) 'The welfare adjustment process: Its stability properties,' International Economic Review, 12: 415-430.

Mantel, R. R. (1974) 'On the characterization of aggregate excess demand,' Journal of Economic Theory, 12: 197-201.

Mas-Colell, A. (1974) 'Continuous and smooth consumers: Approximation theorems,' Journal of Economic Theory, 8: 305-336.

Mas-Colell, A. (1975) 'On the continuity of equilibrium prices in constant-returns production economies,' Journal of Mathematical Economics, 2: 21-33.

Mas-Colell, A. (1977) 'On the equilibrium price set of an exchange economy,' Journal of Mathematical Economics, 4: 117-126.

Mas-ColelI, A. (1985) The theory of general economic equilibrium: A differentiable approach. Cambridge: Cambridge University Press.

Mas-Colell, A. (1986) 'Large substitution effects and the uniqueness of equilibrium,' seminar handout, NBER General Equilibrium Conference, Berkeley.

Mas-Colell, A. (1989) 'On the uniqueness of equilibria once again,' unpublished manuscript, Harvard University. 
Mas-Colell, A. and W. R. Zame (1989) 'Infinite economies,' in W. Hildenbrand and H. Sonnenschein, eds., Handbook of Mathematical Economics, vol. IV. New York: North-Holland, forthcoming.

Merrill, O. H. (1971) 'Applications and extensions of an algorithm that computes fixed points of certain non-empty convex upper semi-continuous point to set mappings,' unpublished manuscript, University of Michigan.

Montrucchio, L. (1987) 'Lipschitz continuous policy functions for strongly concave optimization problems,' Journal of Mathematical Economics, 16: 259-273.

Muller, W. J. and M. Woodford (1988) 'Determinacy of equilibrium in stationary economies with both finite and infinite lived consumers,' Journal of Economic Theory, 46: 255-290.

Nataf, A. (1953) 'Possibilité d'agrégegation dans le cadre de la theorie des choix,' Metroeconomica, 5: $22-30$.

Negishi, T. (1960) 'Welfare economics and existence of an equilibrium for a competitive economy,' Metroeconomica, 12: 92-97.

Ortega, J. and W. Rheinboldt (1970) Iterative solution of nonlinear equations in several variables. New York: Academic Press.

Pareto, V. (1909) Manuel d'economie politique. Paris: Girard and Briere.

Peleg, B. and H. E. Ryder (1972) 'On optimal consumption plans in a multi-sector economy,' Review of Economic Studies, 39: 159-170.

Press, W. H., B. P. Flannery, S. A. Teukolsky, and W. T. Vetterling (1986) Numerical recipes: The art of scientific computing. New York: Cambridge University Press.

Radner, R. (1966) 'Optimal growth in a linear-logarithmic economy,' International Economic Review, 7: 1-33. 
Romer, P. M. (1986) 'Increasing returns and long-run growth,' Journal of Political Economy, 94: 1002-1036.

Rust, J. (1987) 'Optimal replacement of GMC bus engines: An empirical model of Harold Zurcher,' Econometrica, 55: 999-1033.

Ryder, H. E. and G. M. Heal (1973) 'Optimal growth with intertemporally dependent preferences,' Review of Economic Studies, 40: 1-31.

Saari, D. G. (1985) 'Iterative price mechanisms,' Econometrica, 53: 1117-1131.

Saari, D. G. and C. Simon (1978) 'Effective price mechanisms,' Econometrica, 46: 1097-1125.

Samuelson, P. A. (1941) 'The stability of equilibrium,' Econometrica, 9: 97-120.

Samuelson, P. A. (1951) 'Abstract of a theorem concerning substitutability in open Leontief models,' in T.C. Koopmans, ed., Activity analysis of production and allocation. New York: Wiley.

Samuelson, P. A. (1958) 'An exact consumption-loan model of interest with or without the social contrivance of money,' Journal of Political Economy, 6: 467-482.

Santos, M. S. (1989a) 'Differentiability and comparative analysis in discrete-time infinite horizon optimization problems,' unpublished manuscript, Universidad Autonoma de Barcelona.

Santos, M. S. (1989b) 'Smoothness of the policy function in discrete time economic models,' Econometrica, forthcoming.

Santos, M. S. and J. L. Bona (1989) 'On the structure of the equilibrium price set of overlapping-generations economies,' Journal of Mathematical Economics, 18: 209-230.

Sargent, T. J. (1987) Dynamic macroeconomic theory. Cambridge, MA: Harvard University Press.

Scarf, H. E. (1960) 'Some examples of global instability of competitive equilibrium,' International Economic Review, 1: 157-172.

Scarf, H. E. (1967) 'On the approximation of fixed points of a continuous mapping,' SIAM Journal of Applied Mathematics, 15: 1328-1343. 
Scarf, H. E. with the collaboration of T. Hansen (1973) The computation of economic equilibrium. New Haven: Yale University Press.

Scarf, H. E. (1982) 'The computation of equilibrium prices,' in K.J. Arrow and M.D. Intriligator, eds., Handbook of mathematical economics, vol. II. New York: North-Holland.

Scheinkman, J. A. (1976) 'On optimal steady states of n-sector growth models when utility is discounted,' Journal of Economic Theory, 12: 11-30.

Shafer, W. and H. Sonnenschein (1982) 'Market demand and excess demand functions,' in K. J. Arrow and M. D. Intriligator, eds., Handbook of mathematical economics, vol. II. New York: North-Holland.

Shapley, L. S. and M. Shubik (1977) 'An example of a trading economy with three competitive equilibria,' Journal of Political Economy, 85: 873-875.

Shoven, J. B., and J. Whalley (1973) 'General equilibrium with taxes: A computational procedure and existence proof,' Review of Economic Studies, 40: 475-490.

Shoven, J. B. and J. Whalley (1984) 'Applied general equilibrium models of taxation and international trade,' Journal of Economic Literature, 22: 1007-1051.

Smale, S. (1976) 'A convergent process of price adjustment and global Newton methods,' Journal of Mathematical Economics, 3: 1-14.

Sonnenshein, H. (1973) 'Do Walras' identity and continuity characterize the class of community excess demand functions?,' Journal of Economic Theory, 6: 345-354.

Spear, S. E. (1988) 'Growth, externalities, and sunspots,' unpublished manuscript, Carnegie-Mellon University.

Stoer, J. and R. Bulirsch (1980) Introduction to numerical analysis. New York: Springer-Verlag. Stokey, N. L. and R. E. Lucas with E. C. Prescott (1989) Recursive methods in economic dynamics. Cambridge, Mass.: Harvard University Press. 
Streufert, P. A. (1990) 'Stationary recursive utility and dynamic programming under the assumption of biconvergence,' Review of Economic Studies, 57: 79-97.

Szu, H. and R. Hartley (1987) 'Fast simulated annealing,' Physics Letters A, 122: 157-162.

Tauchen, G. (1990) 'Solving the stochastic growth model using quadrature methods and value-function iterations,' Journal of Business and Economic Statistics, 8: 49-52.

Taylor, J. B. and H. Uhlig (1990) 'Solving nonlinear stochastic growth models: A comparison of alternative solution methods,' Journal of Business and Economic Statistics, 8: 1-18.

Todd, M. J. (1976a) The computation of fixed points and applications. New York: Springer-Verlag.

Todd, M. J. (1976b) 'Orientation in complementary pivot algorithms,' Mathematics of Operations Research, 1: 54-66.

Todd, M. J. (1979) 'A note on computing equilibria in economies with activity analysis models of production,' Journal of Mathematical Economics, 6: 135-144.

Traub, J. F. and H. Wozniakowski (1976) 'Optimal linear information for the solution of non-linear equations,' in J. F. Traub, ed., Algorithms and complexity. New York: Academic Press.

Uzawa, H. (1962) 'WaIras' existence theorem and Brouwer's fixed point theorem, 'Economic Studies Quarterly, 13: 59-62.

Vanderbilt, D. and S. G. Louie (1984) 'A Monte Carlo simulated annealing approach to optimization over continuous variables,' Journal of Computational Physics, 36: 259-271.

Varian, H. (1977) 'A remark on boundary restrictions in the global Newton method,' Journal of Mathematical Economics, 4: 127-130.

Wald, A. (1936) 'Uber einige Gleichungssysteme der mathematischen Okonomie,' Zeitschrift fur Nationalokonomie, 7: 637-670; translated as Wald, A. (1951) 'On some systems of equations in mathematical economics,' Econometrica, 19: 368-403. 
Walras, L. (1874) Elements d'economie politique pure. Lausanne: Corbaz; translated by W. Jaffe (1954) Elements of pure economics. London: Allen and Unwin.

Weitzman, M. (1973) 'Duality theory for infinite horizon convex models,' Management Science, 19: 783-789.

Whiteman, C. H. (1983) Linear rational expectations models: A user's guide. Minneapolis: University of Minnesota Press.

Wilson, C. A. (1981) 'Equilibrium in dynamic models with an infinity of agents,' Journal of Economic Theory, 24: 95-111.

Yano, M. (1984) 'The turnpike of dynamic general equilibrium paths and its insensitivity to initial conditions,' Journal of Mathematical Economics, 13: 235-254.

Young, D. M. (1971) Iterative solutions of large linear systems. New York: Academic Press. 